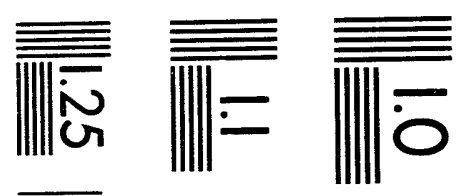

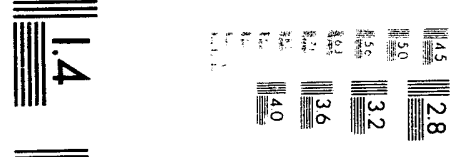

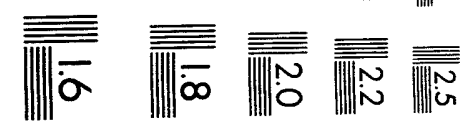



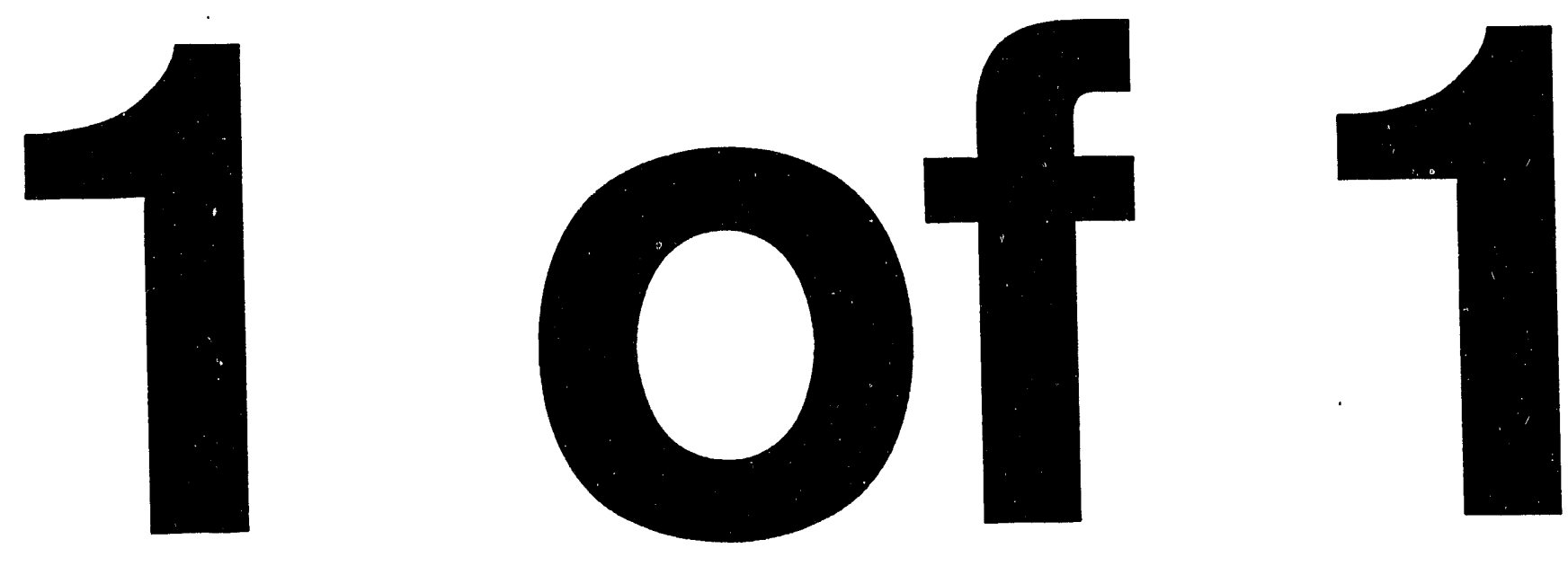
LBL-34255

UC-411

\section{Center for Beam Physics}

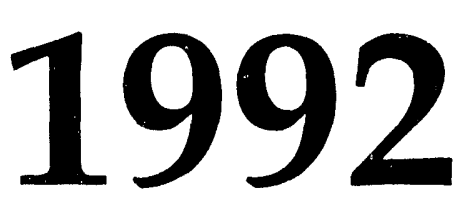

Accelerator and Fusion Research Division

Lawrence Berkeley Laboratory

University of California

Berkeley, California 94720

\section{June 1993}

This work was supported principally by the U.S. Department of Energy under Contract No. DEAC03-76SF00098. Support came from the Director, Office of Energy Research, through the Office of Basic Energy Sciences (Materials Sciences Division) and the Office of High Energy and Nuclear Physics (High Energy Physics Division).

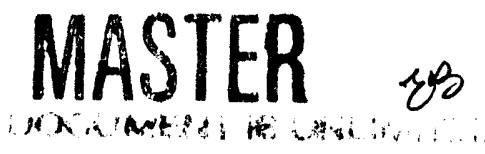


(After PUB-713,

March 1993)

\title{
CENTER
}

FOR

$\boldsymbol{B}_{E A M}$

$\boldsymbol{P}_{\text {HYSICS }}$

\author{
March 1993 \\ Accelerator and Fusion Research Division \\ Lawrence Berkeley Laboratory \\ University of California \\ Berkeley, California 94720
}

Mailing Address:

$\boldsymbol{C}_{\text {ENTER FOR }} \boldsymbol{B}_{\text {EAM }} \boldsymbol{P}_{\text {HYSICS }}$

Mailstop 71-259

Lawrence Berkeley Laboratory

1 Cyclotron Road

Berkeley, CA 94720

Phone: (510) 486-5792

Fax: $\quad$ (510) $486-7981$

E-mail: CBP@lbl.gov 


\section{CONTENTS}

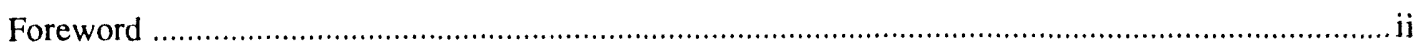

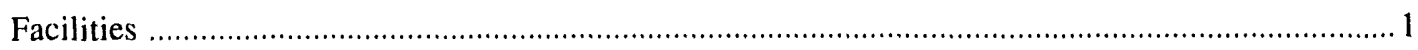

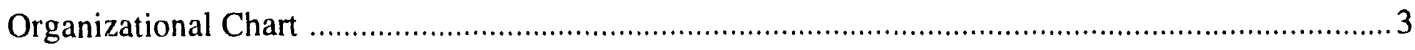

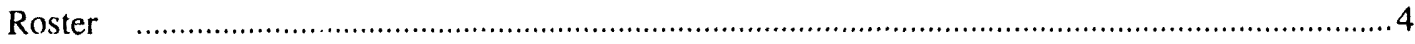

Profiles

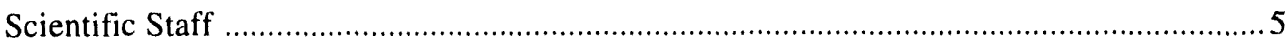

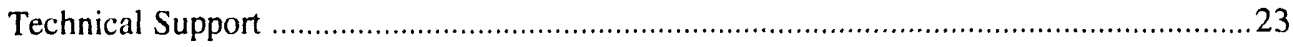

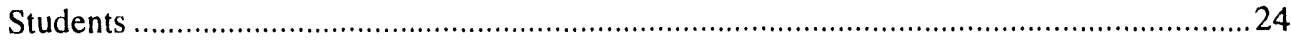

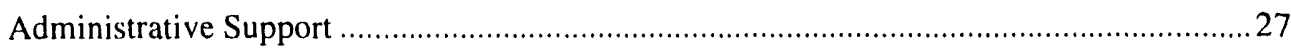

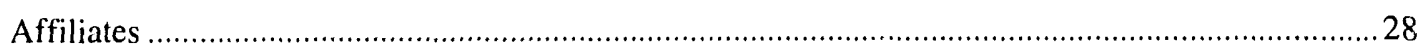

Center Publications (1991-93)

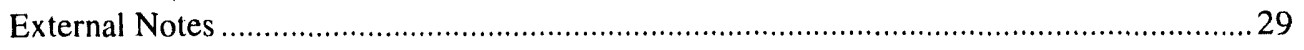

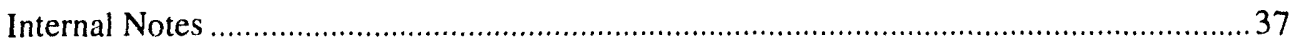

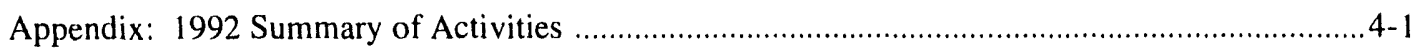

(Reprinted from Chapter 4, Accelerator and Fusion

Research Division: 1992 Summary of Activities,

Lawrence Berkeley Laboratory, University of

California, December 1992, LBL-33377, UC-414) 


\title{
FOREWORD
}

\author{
"Nothing happens unless first a dream" \\ - Carl Sandburg
}

The Center for Beam Physics is a multi-disciplinary research and development unit in the Accelerator and Fusion Research Division of the Lawrence Berkeley Laboratory. At the heart of the Center's mission is the fundamental quest for mechanisms of acceleration, radiation and focussing energy. The Center is dedicated to exploring and investigating the frontiers of the physics of (and with) particle and photon beams. Its primary mission is to promote the science and technology of the production, manipulation, storage and control of systems of charged particles and photons - often in the form of 'beams' with directed energy - as applied to studies of the fundamental structure and processes of the natural world as well as for the very sake of understanding the science of focussing and directing energy. The Center serves this mission via conceptual studies, theoretical and experimental research, design and development, institutional project involvement, external collaborations, association with industry and technology transfer. These activities support exploring the next steps in the development of particle accelerators which are important both for probing the fundamental interactions and for the wide range of disciplines now turning to synchrotron radiation sources and free electron lasers. Accordingly, the program of the Center is not limited to specific programmatic categories of the Department of Energy, but rather serves wide areas of research. The research program of the Center is directly linked with advances in high energy and nuclear physics, condensed matter, material and chemical sciences and the life sciences.

Yet another important mission of the Center is education of students and scientific as well as outside community via graduate instruction, research supervision and pedagogical expositions.

Special features of the Center's program include addressing R\&D issues needing long development time and providing a platform for conception, initiation and support of institutional projects based on 'beams'. The Center brings a significant amount of diverse, complementary and self-sufficient expertise in accelerator physics, synchrotron radiation, advanced microwave techniques, plasma physics, optics and free electron lasers to bear on the forefront $R \& D$ issues in particle and photon beam research. In addition to functioning as a clearing house of ideas and concepts and the necessary related R\&D (e.g. various theoretical and experimental studies in beam physics, nonlinear dynamics, optics and instrumentation), the Center provides core support to laboratory facilities and initiatives e.g. core accelerator physics and systems support to the Advanced Light Source (ALS), technical support for the PEP-II asymmetric B-factory and the LBL proposed Chemical Dynamics Research Laboratory (CDRL) initiative, etc..

The multi-disciplinary programs of the Center are funded by various divisions within the DOE (largely by High Energy and Nuclear Physics and Basic Energy Sciences), as well as laboratory directed R\&D funds. The Center also manages three inhouse research facilities: (i) the Lambertson Beam Electrodynamics Laboratory, (ii) the CBP Laser-Optics Laboratory and (iii) the Beam Test Facility at the A.LS. Formal external collaborations include: (i) SLAC-LBL-LLNL PEP-II studies, (ii) Stanford-LBL-BNLTRW on FEL SCRF technology, (iii) LBL-Stanford on FEL diagnostics, (iv) CEBAF-LBL on IRFEL studies and (v) LBL-Peking University on Photocathode/SCRF technology.

This roster provides a glimpse at the scientists, engineers, technical support, students, and administrative staff that make up this outstanding team. The following pages provide a flavor of our multifaceted activities during 1992.

Swapan Chattopadhyay

Head, Center for Beam Physics 


\section{CENTER FOR BEAM PHYSICS}

\section{Facilities}

\section{Lambertson Beam Electrodynamics Laboratory}

Nurtured, promoted and continually updated over the years by Glen Lambertson of LBL. the laboratory houses, in an environment of controller temperature, various instruments, equipments and apparatus for low-power-level, high-precision RF measurements of beam-handling structures. Inventory includes sophisticated head pulling apparatus, time domain reflectrometry set up, high frequency network and spectrum analyzers, microwave parts and absorbing materials, etc. Also includes a small shop set-up and facilities to perform sophisticated electrodynamic computation of properties of dynamic RF devices.

\section{CBP Laser-Optics Laboratory}

The Laboratory houses lasers, optical components, plasma devices and computers for data acquisition and control for experimental study of optical cavities, optical spectrometers, scaled FEL optics configurations, plasmas, etc.

\section{Beam Test Facility}

The facility, presently under construction, will provide access to a $50 \mathrm{MeV}$ clectron beam from the ALS injector linac, transferred via a magnetic transport line to a specially shiclded experimental vault for various beam-plasma, laser-electron beam scattering and beamRF structure interaction studies.

\section{CBP Dedicated Workstations}

Solbourne 502

Hewlett Packard 375

IBM RS/600)(two)

VAXstation II

\section{CBP Mini-Library}

The library contains selected reference and textbooks on beams, plasmas, lasers, accelerator physics, dynamics, etc., as well as a few technical journals, recent preprints and conference proceedings. It is also used as a mini conference room.

\section{APIARY Conference Room and Microwave Link}

This is a large conference room for seminars and meetings with the special feature of heing connected via a microwave link to SLAC, allowing joint conferences and meetings with the scientists and engineers from SLAC and Stanford University. At present, the room is routinely used for joint LBL-SLAC-LLNL meetings on the PEP-II asymmetric B-factory, elegantly acronymed as APIARY (Asymmetric Particle Interaction Accelerator Research Yard) by LBL physicist A.A. Garren previous to the present project title. It is also used regularly for biweckly Center for Beam Physics seminars. 
CENTER for BEAM PHYSICS

Organization

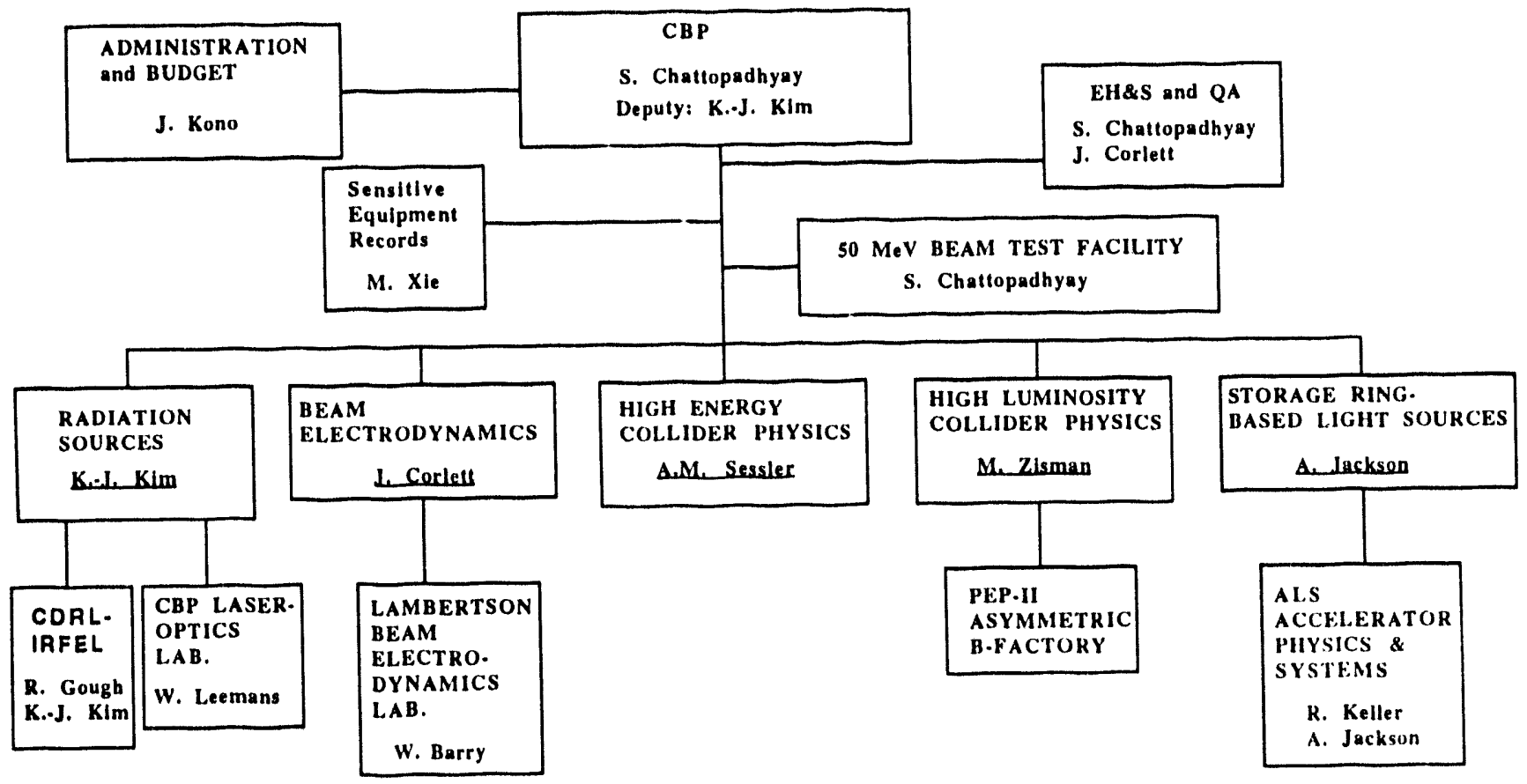




\section{CENTER FOR BEAM PHYSICS}

\section{Roster}

\section{Scientists and Engineers}

BARLETTA, William

BARRY, Walter

BENGTSSON, Johan

BYRD, John

CHATTOPADHYAY, Swapan

CHIN, Yong Ho

CONDE, Manoel

CORLFTT, John

EDIGHOFFER, John

FOREST, Etienne

FURMAN, Miguel

GARREN, Alper

GOLDBERG, David

GOUGH, Richard

JACKSON, Alan

JOHNSON, Jimmic

KELLER, Roderich

KIM, Charles

KIM, Kwang-Je

KWON, SOo-II

LAMBERTSON, Glen

LEEMANS, Wim

LI, Hai

MEDDAHI, Malika

NISHIMURA, Hiroshi

RIMMER, Robert

ROBIN, David

SCHACHINGER, Lindsay

SELPH, Frank

SESSLER, Andrew

VOELKER, Ferdinand

WANG, Changbiao

XIE, Ming

ZHOLENTS, Alexander

ZISMAN, Michael
Technical Support

HARNDEN, C. Warren MASSOLETTI, Dexter SMITHWICK, James

WISE, James

\section{Students}

DUNN, Jason

FIORENTINI, Giulia

GOVIL, Richa

LAMON, Ken

LIDIA, Sicve

WALLACE, Eric

A dministrative Support

CONDON, Martha

KONO, Joy

MARA-ANN, M.

MORETTI, Darlene 


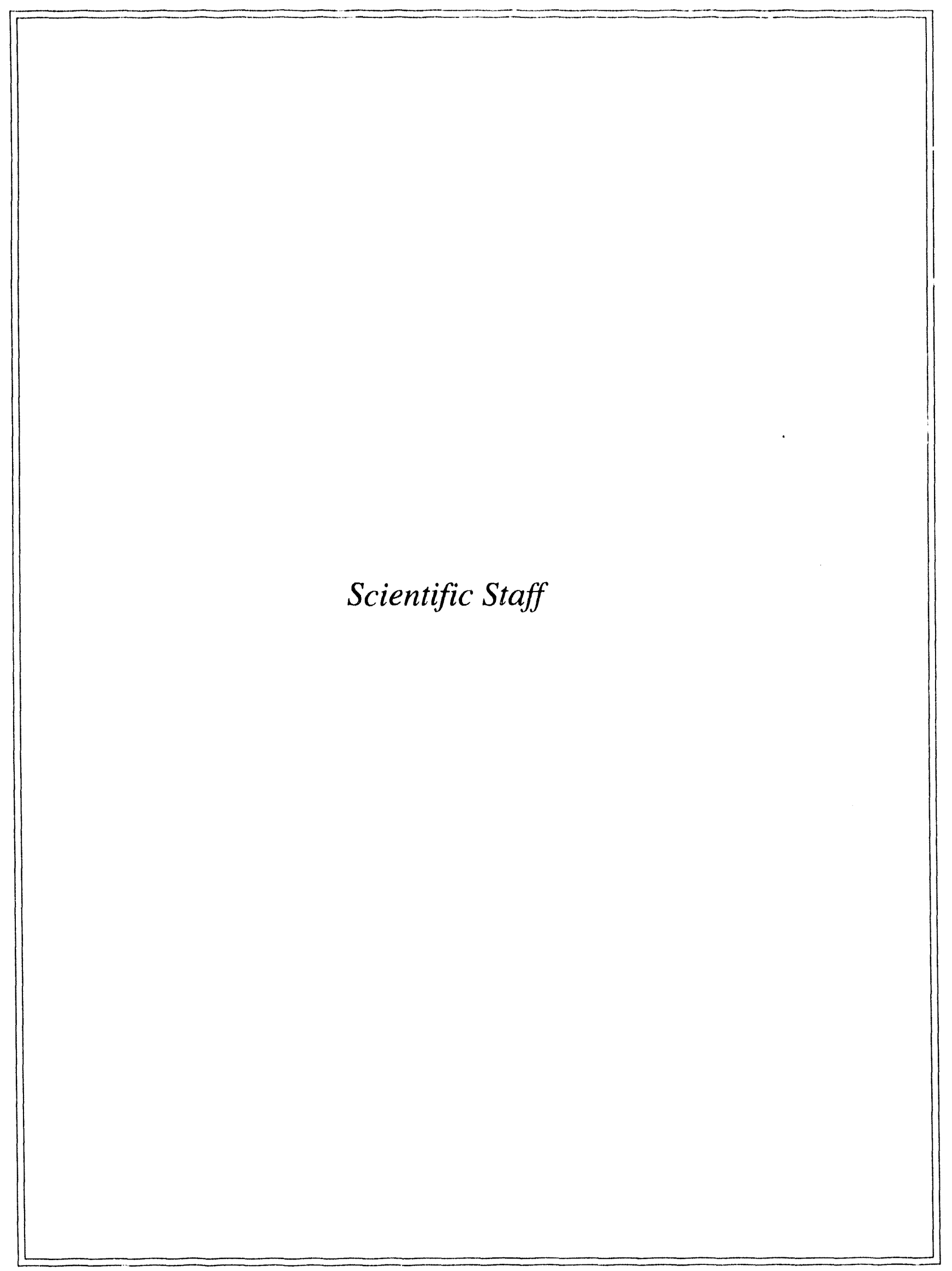




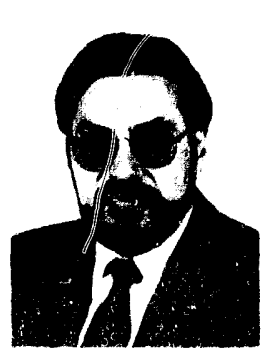

\section{William A. Barletta}

Senior Scientist

Director, Accelerator and

Fusion Research Division

MS 50-149

(510) 486-5501

barletta@lbl.gov

Joined LBL in 1993

Ph.D., Experimental High Energy Physics, Univ. of Chicago (1972). 1989-92: Visiting Professor, Dept. of Physics, UCLA. 1990-93: Assist. Lab. Assoc. Director for Programs at Lawrence Livermore National Laboratory.

Affiliations and honors: Sigma Xi (Yale), Woodrow Wilson Fellow (Univ. of Chicago), member of American Physical Society.

Research interests: colliders at the energy and luminosity frontiers, ultrashort-pulse X-ray sources, radiation processing of chemical and nuclear wastes.

Selected publications: "Luminosity Limitations in Hadron Supercolliders," to be published in Supercolliders and Superdetectors, W. Barletta and H. Leutz, ed., World Scientific, 1993.

"Characteristics of a High Energy $\mu^{+} \mu-$ Collider Based on Electroproduction of Muons" (with A.M. Sessler), LBL Report No. 33613, for submission to Nucl. Instrum. Meth., (Jan. 1993).

"Physically Transparent Formulation of the Free Electron Laser in the Linear Gain Regime" (with A. M. Sessler and L. H. Yu), Nucl. Instrum. Meth. and Proceedings of the 14th International Free Electron Laser Conference, Kobe, Japan, (Aug. 1992).

"Measurements of Photodesorption from Copper Alloys" (W.A. Barletta et al.), Proceedings of the 15th International High Energy Accelerator Conference, Hamburg, Germany, (July, 1992).

"Critical Vacuum Issues for B-factories," Proceedings of International Symposium on "B-factories: State of the Art," Stanford, (April 1992).

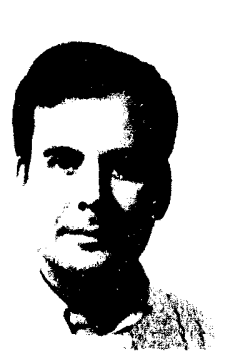

Walter C. Barry

Staff Scientist

MS 71-259

(510) 486-6705

Joined LBL in 1992
M.S., Electrical Engineering, Georgia Institute of Technology, 1982.

Research interests: accelerator instrumentation, theory and applications of electromagnetic and microwave devices in accelerators, coherent transition and diffraction radiation, superconducting RF cavity studies, feedback systems for controlling coupled bunch instabilities in electron storage rings.

Selected publications: "An Autocorrelation Technique for Measuring Sub-Picosecond Bunch Length Using Coherent Transition Radiation," Proceedings of the 1991 Advanced Beam Instrumentation Workshop, KEK National Laboratory for High Energy Physics, Tsukuba, Japan, April 22 24, 1991 .

"A General Analysis of Thin Wire Pickups for High Frequency Beam Position Monitors," Nucl. Instrum Meth. Vol. A301, No. 3, March 15, 1991.

"Characteristic Impedance and Loss Data for a Common Stripline Pickup Geometry" (with S. Y. R. Liu), Nucl. Instrum. Meth. , Vol. A288, Nos. 2, 3, March 15, 1990.

"Perturbation Method for the Measurement of Longitudinal and Transverse Beam Impedance" (with G. Lambertson), Proceedings of the 1987 IEEE Particle Accelerator Conference (March 1987).

"A Broadband Automated, Stripline Technique for the Simultaneous Measurement of Complex Permittivity and Permeability," IEEE Transactions on Microwave Theory and Techniques, Vol. MTT-34, No. 1 (January 1986). 


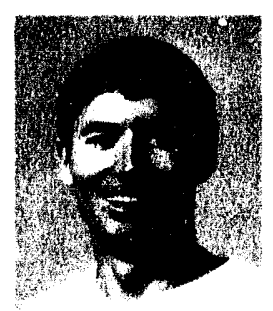

Johan A. Bengtsson

Staff Scientist

MS 71-259

(510) 486-6529

jbengtsson@lbl.gov

Joined LBL in 1989
Ph.D., Physics, MAX-lab, University of Lund, Sweden, 1988.

Research interests: circular accelerators, beam dynamics, beam measurements, signal processing, computer science, control theory.

Selected publications: "Application of Symbolic Computation to the Search of Complicated Primitives: the Example of the Betatron Integrals" (with B. Autin), Comput. Phys. Commun., 48 (1988).

"Application to the Yoshida-Ruth Techniques to Implicit Integration and Multi-Map Explicit Integration" (with E. Forest and M. F. Reusch), Phys. Lett. A, 158 (1991).

"Achromatic and Isochronous Electron Beam Transport for Tunable Free Electron Lasers" (with K.-J. Kim), Nucl. Instr. \& Meth. in Phys. Res. A, 318 (1992).

"Non-Linear Transverse Dynamics for Storage Rings with Applications to the Low-Energy Anti-proton Ring (LEAR) at CERN," CERN 88-05 (1988).

"Absolute and High Precision Measurements of Particle Beam Parameters at CERN Anti-proton Storage Ring LEAR Using Spectral Analysis with Correction Algorithms" (with E. Asseo and M. Chanel), IVth European Signal Processing Conference F-38402 Saint Martin d'Heres, Sept. 5-8, 1988.

"Modeling in Control of the Advanced Light Source" (with E. Forest, H. Nishimura and L. Schachinger), IEEE 1991 Particle Accelerator Conference, San Francisco, California, May 6-9, 1991.

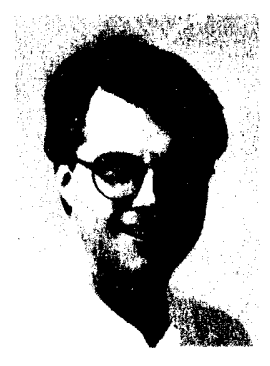

John M. Byrd

Staff Scientist

MS 71-259

(510) 486-6329

jbyrd@lbl.gov

Joined LBL in 1991
Ph.D., Physics, Cornell University, 1992.

Research interests: RF aspects of accelerators, coupled-bunch instabilities and feedback systems.

Selected publications: "Progress on PEP-II Multibunch Feedback Kickers"' (with J. Johnson, G. Lambertson, F. Voelker), Proc. SLAC B Factory Workshop (June 1992).

"Longitudinal Beam Response Measurements at CESR," Proc. of the 199I IEEE Particle Accelerator Conference (May 1991).

"Measurement of Octupole-induced Decoherence at CESR" (with D. Sagan), Proc. of the 1991 IEEE Particle Accelerator Conference (May 1991).

"Cornell Synchrotron Tune Correction" (with C. Dunnam and R. Meller), Proc. of the 1990 Accelerator Instrumentation Conference (October 1990).

"An Uncoupled, Round Beam, Electron Accelerator Lattice" (with D. Sagan, and R. Talman), Proc. of the 3rd Advanced ICFA Beam Dynamics Workshop (June 1989). 


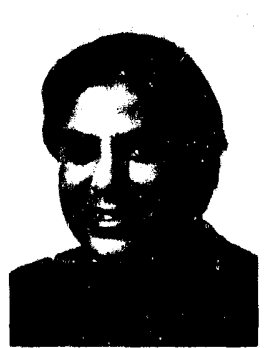

\author{
Swapan Chattopadhyay \\ Senior Scientist \\ Head \\ Center for Beam Physics \\ MS 71-259 \\ (510) 486-7217 \\ chapon@lbl.gov \\ csa::chapon \\ Joined LBL in 1976
}

Ph.D., Physics, University of California, Berkeley, 1982. Attaché Scientifique, CERN, Geneva, Switzerland, 1982-84. Guest lecturer, UC Berkeley, 1987. Visiting Prof., Univ. of Illinois at UrbanaChampaign. 1991.

Affiliations: Editor-in-chief, Particle Accelerators (Western Hemisphere); Member: American Physical Society (APS), American Association for the Advancement of Science (AAAS), International Committee on Future Accelerators (ICFA), Advisory Board to International Linac Conferences, Advisory Committee to PEP-II Project. National Scholar (1967) and National Science Talent Scholar (1967-72), Govt. of India.

Research interests: particle and photon beam physics; synchrotron radiation; free electron lasers; beamplasma physics; nonlinear dynamics; collider physics; novel accelerators.

Selected publications: "Generation of Femtosecond X-Rays by $90^{\circ}$ Compton Scattering" (with K-J. Kim and C. Shank), LBL-33074, to be published in Nucl. Instr. Methods in Phys. Res.

"Physics and Design Issues of Asymmetric Storage Ring Colliders as B-Factories," Particle Accelerators, Vol. 30, (1990).

"Feasibility Study of a Storage Ring for a High-Power XUV Free Electron Laser" (with J.J. Bisognano et al.), Particle Accelerators, Vol. 18, p. 223, (1986).

"Some Fundamental Aspects of Fluctuation and Coherence in Charged Particle Beams in Storage Rings," AIP Conf. Proc. Series, No. 127, p. 467. (1985).

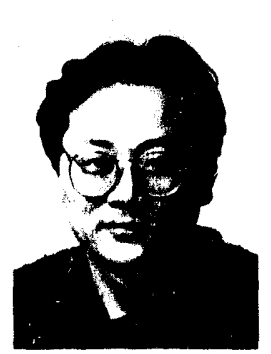

\author{
Yong Ho Chin \\ Staff Scientist \\ MS B71H \\ (510) 486-5614 \\ yongho@lbl.gov \\ Joined LBL in 1988
}

Ph.D., Physics, The University of Tokyo, 1984.

Major awards: Japan Accelerator Society Annual Award.

Research interests: free electron laser, calculation of wake fields.

Selected publications: "User's Guide for New ABCI, Version 6.2 (Azimuthal Beam Cavity Interaction)," CERN Report CERN SL/92-49 (AP) and LBL-33091 (1992).

"Three-Dimensional Theory of Small-Signal, HighGain Free Electron Laser Including Betatron Oscillations" (with K.-J. Kim and M. Xie), to be published in Phys. Rev. A, 46, p. 6662 (1992); Lawrence Berkeley Laboratory Report LBL-32329 (1992).

"Renormalized Theory of Beam-Beam Interaction in Electron-Positron Colliders," in Proc. of the 3 rd Advanced ICFA Beam Dynamics Workshop on BeamBeam Effects in Circular Colliders, edited by I. Koop, and G. Tumaikin Akademgorodok, Novosibirsk, USSR, pp. 69-75 (May 1989).

“User's Guide for New MOSES Version 2.0 (Modecoupling Single Bunch Instability in an Electron Storage Ring)," CERN Report CERN/LEP-TH/88-05 (1988).

"Nonlinear Perturbation Approach to Bunch Lengthening and Blow-up of Energy Spread" (with K. Yokoya), Nucl. Instrum. Methods, 226, p. 223 (1984).

"Analytical Approach to the Overshoot Phenomenon for a Coasting Beam in Particle Accelerators" (with K. Yokoya), Phys. Rev. D, 28, p. 2141 (1983). 


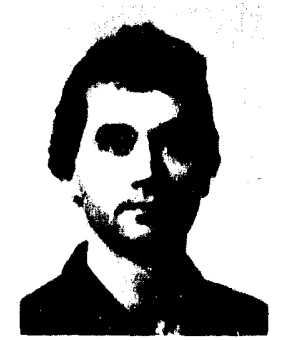

Manoel E. Conde

Staff Scientist

MS B71H

(510) 486-5076

conde@lbl.gov

Joined LBL in 1992

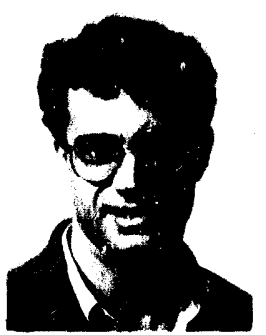

John N. Corlett

Staff Scientist

Group Leader (acting)

Beam Electrodynamics

Group

MS 71-259

(510) 486-5228

jnc@bcl.lbl.gov

Joined LBL in Dec. 1991
Ph.D., Physics, Massachusetts Institute of Technology, 1992.

Research interests: free-electron lasers, particle accelerators and plasma physics, studies of photocathode RF guns.

Selected publications: "Amplification and Superradiant Emission from a $33.3 \mathrm{GHz}$ Free Electron Laser with a Reversed Axial Guide Magnetic Field" (with G. Bekefi), IEEE Trans. Plasma Sci., 20, p. 240 (1992).

"Experimental Study of a $33.3 \mathrm{GHz}$ Free Electron Laser Amplifier with a Reversed Axial Guide Magnetic Field" (with G. Bekefi), Phys. Rev. Lett., 67, p. 3082 (1991).

"Shape of the Plasma Boundary in TBR" (with R. M. O. Galvao et al.), Rev. Bras. Fis., 17, p. 109 (1987).
BSc, Physics, Liverpool University, 1983. Microwave engineer, EEV Co. Ltd, 1983-1986. Accelerator physicist, Daresbury Laboratory, U.K., 1986-1991.

Research interests: monochromatic RF structures, beam coupling impedance, feedback systems, bunched beam instabilities.

Selected publications: "Measurements of the Higher Order Modes of the ALS $500 \mathrm{MHz}$ Accelerating Cavities" (witl. J. Byrd), to be presented at the Particle Accelerator Conference, Washington, DC (May 1993).

"Impedance Measurements of Components for the ALS" (with R. Rimmer), to be presented at the Particle Accelerator Conference, Washington, DC (May 1993).

"New Injection Kicker Magnets for the Daresbury SRS" (with J.A. Clarke), Proc. 3rd European Particle Accelerator Conference, Berlin (March 1992).

"Higher Order Modes in the SRS $500 \mathrm{MHz}$ Accelerating Cavities," Particle Accelerator Conference, Chicago (March 1989).

"SRS-2 Performance and Achievements" (with V.P. Suller et al.), Particle Accelerator Conference, Chicago (March 1989).

"Beam Instability Characteristics of the Daresbury SRS" (with M.W. Poole, V.P. Suller and J.S. MacKay), European Particle Accelerator Conference, Rome (June 1988) 


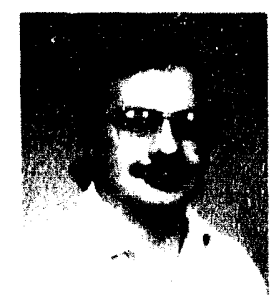

John A. Edighoffer

Staff Scientist

MS B71H

(510) 486-5107

jedig@lbl.gov

Joined LBL in Aug.1991
Ph.D., Applied Physics, Stanford University, 1981.

Ten years at TRW doing FEL research.

Research interests: free electron lasers, optical diagnostics, photocathodes, superconducting RF. accelerator physics and modeling, accelerator diagnostics; CDRL FEL Conceptual Design, Stanford/ LBL/BNL superconducting RF collaboration, Stanford/ LBL FEL diagnostics collaboration; LBL/CEBAF FEL/ RF photocathode collaboration; hole out-coupling scaled FEL bench top experiments.

Selected publications: "First Operation of a Tapered Wiggler Free Electron Laser Oscillator" (with S.W. Fornaca, G.R. Neal, C. Hess, H.A. Schwettman and T.I. Smith), J. Appl. Phys. (1983).

"Energy Measurement of the Electron Beam Beyond the PALADIN Wiggler" (with T.J. Orzechowski, P. Lee, T.E. Smith, Y.P. Chong, A.C. Paul and J.T. Weir), Proc. of the 1/th FEL Conf. (Sept. 1989).

"Visible Free-Electron Laser Oscillator (Constant and Tapered Wiggler)" (with G.R. Neil, S. Fornaca, H.R. Thompson, Jr., T.I. Smith, H.A. Schwettman, C.E. Hess, J. Frisch and R. Rohatgi), (with H. Boehmer, M.Z. Caponi, S. Fornaca, J. Munch, G.R. Neil, B. Saur and C. Shih), J. Appl. Phys. (June 1987).

"Free Electron Laser Small Signal Gain Measurement at $10.6 \mathrm{~mm}, "$ Appl. Phy. Let. (1982).

"Observation of Inverse Cerenkov Interaction between Free Electrons and Laser Light," (with W.D. Kimura, R.H. Pantell, M.A. Piestrup, and D.Y. Wang), $P h$ y. Rev. A, Vol. 23, No. 4 (April 1981).

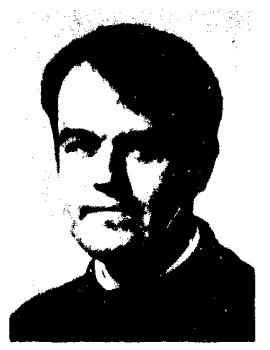

Etienne Forest

Staff Scientist

MS 71-259

(510) 486-7215

etienne@lbl.gov

Joined LBL in 1985
Ph.D., Physics, University of Maryland, 1984.

Research interests: nonlinear dynamics in accelerators, perturbation theory and other approximate methods for accelerator maps.

Selected publications: "The UCLA $\phi$ Factory Collider" (with A. Amiry, C. Pellegrini and D. Robin), to be submitted to Phys. Rev. (1993).

"Construction of Symplectic Maps for Non-linear Motion of Particles in Accelerators" (with J.S. Berg, R.L. Warnock and R.D. Ruth), submitted to Phys. Rev. (1993).

"Sixth Order Lie Group Integrator," J. Comp. Phys. (1992).

"Symplectic Integration in Complex Wigglers" (with K. Ohmi), KEK Report 92-14 (1992).

"A Contemporary Guide to Beam Dynamics" (with K. Hirata), KEK Report 92-12(1992).

"Dynamic Aperture Study for the Duke FEL Storage Ring" (with Y. Wu, V.N. Litvinenko and J. Madey), submitted to the Fourteenth Int'। FEL Conference in Kobe, Japan, August 23-18, 1992, to appear in a special issue of Nuclear Instruments and Methods, Section A, Elsevier North Holland Science Publishers B.V.

"The Absolute Bare Minimum for Tracking in Small Rings" (with M. Reusch, D. Bruhwiler and A. Amiry), submitted to Part. Accel. (1992).

"Application of the Yoshida-Ruth Techniques to Implicit Integration and Multi-Map Explicit Integration" (with M. Reusch and J. Bengtsson), Phys. Lett. A (1991). 


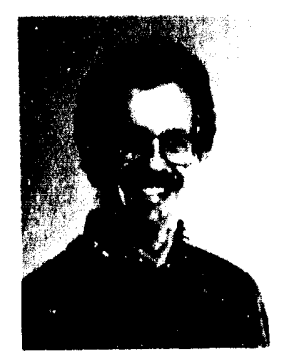

\section{Miguel A. Furman}

Staff Scieritist

MS B7IH

(510) 486-6443

miguel@lbl.gov

Joined LBL in 1984
Ph.D., Theoretical Particle Physics, University of California, Santa Cruz, 1977. Joined LBL/CBP in August, 1984. Worked "on loan" for the SSC Central Design Group (1984-1989), and then for the SSC Laboratory (1989-1990). Since 1990, working fulltime at CBP on the PEP-II project.

Research interests: beam-beam interaction: longitudinal phase space management and matching in chains of accelerators; space-charge effects.

Selected publications: "Beam-Beam Diagnostics from Closed-Orbit Distortion" (with Y-H Chin, J. Eden, W. Kozanecki, J. Tennyson and W. Ziemann), to be published in the Proceedings of the 15th Intl. Conf. on High-Energy Accelerators, Hamburg, July 1992.

"Beam-Beam Issues in Asymmetric Colliders," invited talk, Proc. of the B Factories: State of the Art,

Stanford, California, April 1992, p. 109.

"RAMPRF: a Program for Synchronous Acceleration," Proc. 1991 Particle Accelerator Conference, San Francisco, p. 300 (May 1991).

"Hourglass Effects for Asymmetric Colliders," Proc. 1991 Particle Accelerator Conference, San Francisco, p. 422 (May 1991).

"A Possible Symplectic Coherent Beam-Beam Model" (with A. W. Chao and K.Y. Ng), Proc. European Particle Accelerator Conference, Rome, p. 684 (June 1988).

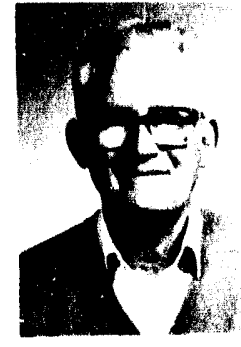

Alper Garren

Senior Scientist

MS B7IH

(510) 486-6574

garren@lbl.gov

Joined LBL in 1955

Ph.D., Physics, Carnegie Institute of Technology, 1955.

Accelerator theorist with contributions to design of many accelerators and their lattices, e.g. Bevatron, FNAL, PEP, BNL/CBA, ALS, PEP-II, SSC, etc. Author of the lattice program SYNCH. Also contributed to heavy ion fusion, magnetic fusion with mirrcr machines, spiral-ridge cyclotrons (e.g. 88" Cyclotron at LBL) and to the Electron Ring Accelerator Study.

Selected publications: "SYNCH Users Guide" (with A.S Kenney, E.D. Courant, A.D. Russell, M. Syphers), SSCL-MAN-0002 (1993).

"APIARY B-Factory Separation Scheme" (with M. Sullivan), LBL-PUB-30730 (May 1991).

"APIARY B-Factory Lattice Design" (with M.H.R. Donald), LBL-PUB-30665 (May 1991).

"Low Momentum Compaction Lattice Study for the SSC Low Energy Booster" (with E.D. Courant and U. Wienands), Proc. of 1991 Particle Accelerator Conference (May 1991).

"Site-Specific Conceptual Design of the Superconducting Supercollider," SSCL-SR-1056 (July 199()).

"An Asymmetric B-Meson Factory at PEP" (A.A. Garren et al.), Proc. of 1989 Particle Accelerator Conference, Chicago.

"Thin Lens Optics With Space Charge," Proc. of 7th Int. Conf. on High Energy' Accelerators, Yerevan, USSR, UCRL-19313, (1969).

"Lattice Of The Nal Proton Synchrotron," Proc. of 1969 Particle Accel. Conference.

"Orbit Dynamics in the Spiral-Ridged Cyclotron" (with Lloyd Smith), UCRL-8598 (1959). 


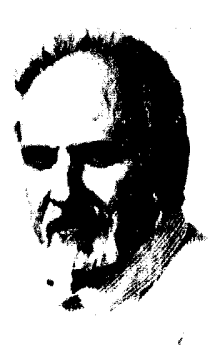

David A. Goldberg

Staff Scientist

MS 71-259

(510) 486-7222

dag@lbl.gov

Joined LBL in 1980

Ph.D., Nuclear Physics, Johns Hopkins University, 1967.

Research interests: beam instrumentation and feedback, beam impedance measurements, stochastic cooling.

Selected publications: "Higher-Order Mode-Damping Studies on PEP-II B-Factory RF Cavity" (with R.A. Rimmer et al.), contribution to 1992 European Particle Accelerator Conference.

"Dynamic Devices: A Primer on Pickups and Kickers" (with G.R. Lambertson), in Physics of Particle

Accelerators, M. Month and M. Dienes, eds. (1992).

"Modes of Elliptical Waveguides: a Correction" (with L.J. Laslett and R.A. Rimmer), IEEE Trans. on Microwave Theory and Techniques, 38 (1990).

"Successful Observation of Schottky Signals at the Tevatron Collider" (with G.R. Lambertson), Particle Accelerators, 30 (1990).

"Improving the Performance of Power-Limited Stochastic Cooling Systems" (with G.R. Lambertson), Particle Accelerators, 30 (1990).

"Beam Impedance Measurements on the ALS Curved Sector Tank" (with R.A. Rimmer et al.), contribution to 1990) European Particle Accelerator Conference.

"A High-Frequency Schottky Detector for Use in the Tevatron Collider" (with G.R. Lambertson), Proceedings of 1990 Workshop in Accelerator Instrumentation.

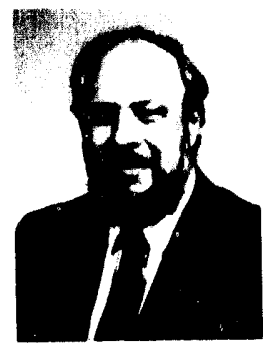

Richard A. Gough

Senior Scientist

MS 50-149

(510) 486-4573

RAGough@LBL

Joined LBL in 1970

Program Head

Special Projects, AFRD

Ph.D., Nuclear Physics, McMaster University, 1970.

Research interests: design, construction, and management of accelerator facilities, conceptualization and development of accelerator facilities with applications to the scientific community.

Selected publications: "Design of a Superconducting Linear Accelerator for an Infrared Free Electron Laser of the Proposed Chemical Dynamics Research Laboratory at LBL" (with S. Chattopadhyay, R. Byrns, R. Donahus, J. Edighoffer, E. Hoyer, K.-J. Kim, W. Leemans, J. Staples, B. Taylor, and M. Xie), 16th International Linac Conference, Ottawa, Ontario, Canada, August 23-28, 1992.

"Design Overview of Highly Stable Infrared Free Electron Laser at LBL" (with K.-J. Kim, M. Berz. S. Chattopadhyay, J. Edighoffer, C. Kim, A. Kung, W. Stein, and M. Xie), Proc. of Twelfth International Free Electron Laser Conference, Paris, France, September 17-21, 1990.

"Medical Heavy Ion Accelerator Proposals," Proc. of 1985 Particle Accelerator Conference, Vancouver, B.C., Canada, p. 3282 (May 1985).

"Performance of the Oxygen Injector for the CERN Linac I" (with B. Wolf, K. Leible, P. Spädtke, J. Klabunde, B. Langenbeck, N. Angert, J. Staples, R. Caylor, D. Howard, R. MacGill, J. Tanabe, C. Hill, P. Tetu, M. Weiss, and R. Geller), Nucl. Instr. and Methods, A258 (1987). 


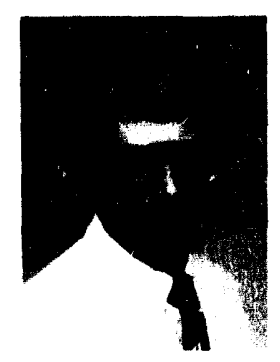

\author{
Alan Jackson \\ Staff Scientist \\ Group Leader \\ ALS Accelerator Group \\ MS 80-101 \\ (5I0) 486-6752 \\ ajackson@lbl \\ Ibl::ajackson \\ Joined LBL in 1984
}

BA (Hons), Physics, Lancaster University, 1968. 1968-84: Scientific Ofticer at Daresbury Nuclear Physics Laboratory, U.K. 1984-present: At LBL, member of the team that designed and commissioned the third generation Advanced Light Source.

\section{Affiliations: Member APS and AAAS}

Research interests: design, construction and operation of synchrotron radiation sources; fourth generation synchrotron radiation source.

Selected publications: "Ideas for Future Synchrotron Light Sources" (A. Jackson et al.), presented at the Third European Accelerator Conference, Berlin, Germany, March 1992, and to be published in the proceedings.

"The Challenges of Third Generation Synchrotron Light Sources," Synchrotron Radiation News, Vol. 3, No. 3, pp. 13-20 (May-June 1990).

"The Effect of Insertion Devices on the Behavior of the ALS," (A. Jackson et al.), IEEE Trans. Nuc. Sci., IEEE 89CH2669-0 (1989).

"A Comparison of the Chasman-Green and Triple Bend Achromat Lattices," Particle Accelerators, Vol. 22, No. 2 (1987).

"Feasibility Study of a Storage Ring for a High Power XUV Free Electron Laser" (with J. Bisognano et al.), Particle Accelerators, Vol. 18 (1986).

"The NINA Polarized Photon Beam," Nucl. Instrum. Meth., 129 (1975).

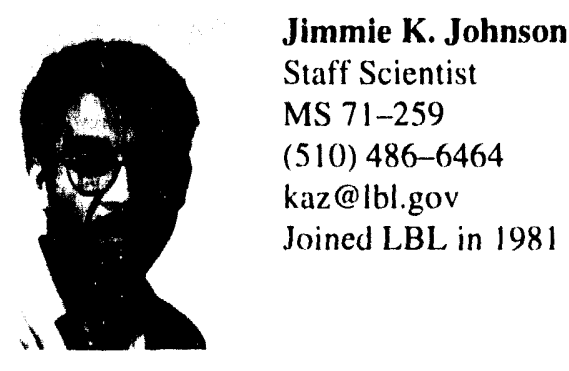

B.S., Electronics Engineering, University of California, Davis, 1981.

Affiliations: IEEE, LBL representative to the National Consortium for Graduate Degrees for Minorities in Science and Engineering, Inc. (GEM)

Research interests: microwave technology with accelerator applications, computer-aided engineering, multi-bunch feedback systems.

Selected publications: "Progress on PEP-II Multibunch Feedback Kickers" (with J. Byrd, G. Lambertson, F. Voelker), Proc. SLAC B Factory Workshop, June 1992).

"Novel Electrode Design for a 4-8 GHz Stochastic Cooling System" (with D. Goldberg, G. Lambertson, F. Voelker), Bull. Am. Phys. Soc., 33, p. 1025 (1988).

"Power Combiners/Dividers for Loop Pickup and Kicker Arrays for FNAL Stochastic Cooling Rings (with R. Nemetz), 1985 Particle Accelerator Conference. Vancouver, B.C., Canada, May 13-15, 1985, IEEE Trans. Nucl. Sci., NS-32, p. 2171 (October 1985).

"An Array of 1 to $2 \mathrm{GHz}$ Electrodes for Stochastic Cooling (with F. Voelker and T. Henderson), 1983 Particle Accelerator Conference, Santa Fe, NM, March 21-23, 1983. 


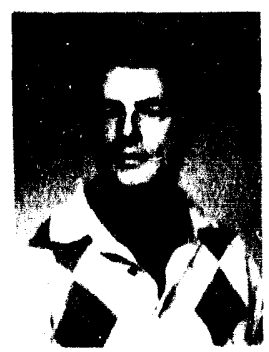

\section{Roderich Keller}

Staff Scientist

Deputy Group Leader

ALS Accelerator Group

Section Leader

ALS Accelerator Physics

MS 8()-101

(510) 486-5223

rkeller@lbl.gov

Joined LBL in 1988

Dr. rer. nat., Experimental Physics, University of Kiel, Germany, 1973.

Awards: Three patents on ion sources and components.

Research interests: particle accelerators; ion sources for accelerators and industrial applications.

Current activities: Commissioning of the ALS (Advanced Light Source) electron accelerators, a 50) $\mathrm{MeV}$ linac, a $1.5 \mathrm{GeV}$ bonster synchrotron, and a 1-1.9 $\mathrm{GeV}$ storage ring. Evaluation of magnetic field data for the accelerators under construction. Definition of survey and alignment procedures, creation of ideal component data, and evaluation of survey data for the ALS accelerattors.

Selected publications: "Survey and Alignment of the Advanced Light Source in Berkeley." Nucl. Instr. Meth. in Phys. Research B56/57, p. 422 (1991).

"Magnetic Data Analysis for the ALS Lattice Magnets," IEEE \#9/CH3038-7. p. 2113(1991).

"Study of a 'Relaxed' ALS Storage Ring Lattice," Second European Particle Accelerator Conf., Nice, France (1990).

"Ion Extraction Systems: Optics and Design," Nucl. Instr. Meth. in Phys. Research A298, pp. 247-254 (1990).

"High Current Gaseous ton Sources" in: I.G. Brown, ed., The Physics and Technology of Ion Sources, p. 151, John Wiley, NY (1989).

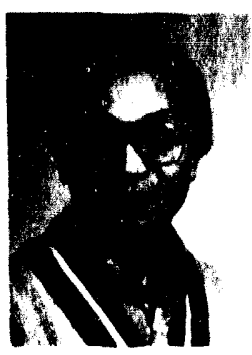

Charles H. Kim

Staff Scientist

MS 8()-101

(510) $486-7218$

ckim (acsa3.lbl.gov

Joined LBL in 1978

Ph.D., Plasma Physics, University of California, Los Angeles, 1974.

Awards: Fannie and John Hertz Foundation Fellow

Research interests: Accelerators, if linac, synchrotron, storage ring, accelerator diagnostics instrumentation, linac simulations.

Selected publications: "Performance of the AIS Injector," to be published in the IEEE Particle Accelerator Conference (1993).

"Advanced Light Source Instrumentation Overview," Proceedings of the 1992 Accelerator Instrumentation Workshop.

"Commissionning lixperiences of the AIS Booster Synchrotron." Proceedings of the IEEE Particle" Acrelerator Conference, p. 2691(1991).

"Dynamic Aperture of the ALS Booster Synchrotron." Proceedings of the IEEE Particle Accelerator Conference, p. 1328 (1989).

"Simulation of Emittance Growth in the ALS PreInjector, "Linear Accelerator Conference Procesedings. p. $427(1988)$.

"Design of a Bunching System for a High-Intensity Electron Linac," Proceedings of the European Particle Conference. p. 86.3(1988).

"Development of Heavy Ion Induction Linear Accelerators as Drivers for Inertial Confinement Fusion," Proceedings of the European Particle Conference, p. 1521(1988). 


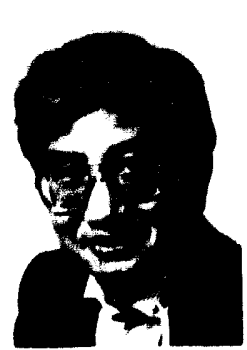

Kwang-Je Kim

Senior Scientist

Deputy Head,

Center for Beam Physics

Lecturer, UCB Phys. Dpt

MS 71-259

(510) 486-7224

kwangje@lbl.gov

Joined L,BL in 1978

Ph.D., Elementary Particle Physics, University of Maryland, 1970. Visiting Scientist, SLAC 1970-73; Max Planck Inst. für Phys. and Astrophys., 1973-75; Univ. of Mainz, 1975-78.

Affiliations: American Physical Society, Int'l FEL, Program Committee (1993), Int'l Advisory Committee for Pohang Light Source.

Research interests: novel $\mathrm{x}$-ray generation, free electron lasers, synchrotron radiation optics, highbrightness electron beams.

Selected publications: "Generation of Sub-Picosecond X-rays by $90^{\circ}$ Compton Scattering" (with S.

Chattopadhyay and C.V. Shank), LBL-33(074.

"Stability and Performance of CDRL-FEL" (with M. Xie), Nucl. Instr. Methods, A304, p. 146 (1991).

"Spectral Bandwidth in FEL Oscillators," Phys. Rev. Lett., 66, p. 2746 (1991).

"RF and Space Charge Effects in Laser-Driven RF Electron Guns," Nucl. Instr. Meth., A275, p. 201 (1989)

"Brightness, Coherence and Propagation Characteristics of Synchrotron Radiation," Nucl. Instr. Meth., A246, p. 71 (1986).

"Three-Dimensional Analysis of Coherent Amplification and Self-Amplified Spontaneous Emission in Free Electron Lasers," Physical Review Letters, 57, p. 1871 (1986).

"A Synchrotron Radiation Source with Arbitrarily Adjustable Elliptical Polarization," Nucl. Instr. Meth., 219 . p. 425 (1984).

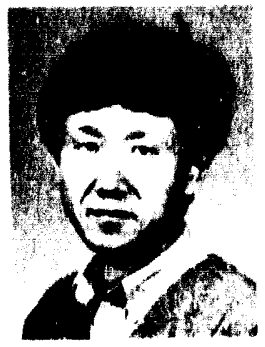

\section{Soo-Il Kwon}

Visiting Researcher

Kyonggi University

Suwon, Korea

MS B $71 \mathrm{H}$

(510) 486-5470

sooil@Ibl.gov
Ph.D., Nuclear Physics, Sung Kyun Kwan University, Korea, 1988.

Research interests: radiation detection and measurement, radiation damage, free electron lasers, laser-driven photocathode electron source.

Selected publications: "Electron Spin Resonance of Gamma-irradiated Single Crystal of L-Alanine," Kor. Appl. Phys., Vol. 6, No. 3 (1993).

"ESR Study of the Proton-Deuteron Exchange Reaction in Irradiated L-Alanine," Kor. Appl. Phys., Vol. 6, No. 3 (1993).

"A Study on the Fabrication of the BGO Scintillation Detector and Its Gamma-Ray Spectroscopic Characteristics," Kor. Appl. Phys., Vol. 4, No. I (1991).

"The Effects of Scintillator Shape and Surface Treatment on the Light Output of BGO Detector," Kor. Appl. Phys., Vol. 3, No. 4 (1990).

"Characteristics of BGO Scintillation Detector Using Silicon Photodiodes," J. Kyonggi Univ., Vol. 21 (1989). 


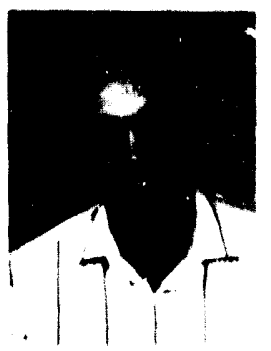

\section{Glen R. Lambertson}

Senior Scientist

MS 71-259

(510) 486-7205

lambertson@lbl.gov

Joined LBL in 1951
M.S., Physics, University of California, Berkeley, 1951.

Award: U.S. Particle Accel. School 1991 Prize for Achievement in Accelerator Physics and Technology.

Current research: particle beam electrodes, stochastic beam cooling, feedback stabilization of beam instabilities.

Selected publications: "Dynamic Devices, A Primer on Pickups and Kickers" (with Goldberg), AIP Conf. Proc. 249, p. 537 (1992).

"Higher Order Mode Damping Studies on the PEP-II B-Factory RF Cavity" (with Rimmer, Goldberg. Voelker, Kroll, Pendleton, Schwarz, Adams, and DeJong), 3rd European Particle Accel. Conf. (1992).

"Transverse Feedback in a 10) TeV Storage Ring." Proc. of 19th Workshop on Maximizing Luminosity of Hadron Colliders at $180 \mathrm{TeV}$. Erice, Italy (1991).

"Control of Coupled-Bunch Instabilities in HighCurrent Storage Rings," invited paper, 199/ IEEE Particle Accelerator Conference, 4. p. 2537 (1991).

"Higher Order Mode Damping in a Pill Box Cavity" (with Rimmer and Voelker), 1991 IEEE Particle Accel. Conf., 2, p. 687 (1991).

"Techniques for Beam Impedance Measurements Above Cutoff" (with Jacob, Rimmer and Voelker), 2 nd European Particle Accel. Conf., p. 1049 (June 1990).

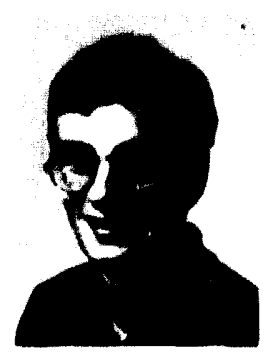

Wim J.eemans

Staff Scientist

MS 71.259

(.510) $486-7788$

leemans(älbl.gov

Joined L.BI. in 1991

Ph.D., Electrical Engineering, University of California, Los Angeles, 1991.

Major awards: American Physical Society Simon Ramo Award 1992.

Research interests: beam-plasma interaction, generation of light, non-linear optics, non-linear dynamics, study of plasma lens focusing, generation of short pulse X-rays through inverse compton scattering. advanced optical diagnostics and resonators for FEL's.

Selected publications: "Ultrahigh Gradient Acceleration of Injected Electron by Laser-Excited Relativistic Electron Plasma Waves" (with C.E. Blayton, K.A. Marsh, A. Dyson, M. Everett, A. Lal, R. Willians and C. Joshi), Physical Review Letters, 10, p. 37 (1993).

"Non-linear Dynamics of Driven Relativistic Electron Plasma Waves" (with C. Joshi, W. B. Mori, C. E. Clayton and T. W. Johnston), Physical Review A, 46, p. 15 (September 1992).

"Experiments and Simulations of Tunnel-lonized Plasmas" (with C. E. Clayton, W. B. Mori, K. A. Marsh, P. K. Kaw, A. Dyson and C. Joshi), Physical Review A. 46, pp. 1091-1105 (1992).

"Plasma Physics Aspects of Tunnel-lonized Gases" (with E. Clayton, W. B. Mori, K. A. Marsh, A. Dyson, and C. Joshi), Phssical Review Letters, 68, pp. 321-324 (1992).

"Stimulated Compton Scattering from Pre-formed Underdense Plasmas" (with C. E. Clayton, K. A. Marsh and C. Joshi), Physical Review Letters, 67, pp. 1434$1437(1991)$. 


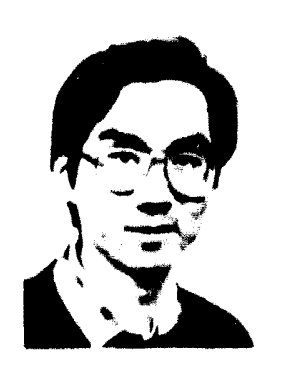

Hai LI

Staff Scientist

MS B7IH

(510) $486-6.572$

hivi कlbl.gov

Joined L.BL in 1993

Ph.D., Physics, University of Maryland at College Park. 1993.

Research interests: analytical and numerical studies of space charge and high frequency electromagnetic problems related to microwave devices such as gyrotrons, relativistic klystrons and FEL.

Selected publications: "Space Charge Instabilities in Gyrotron Beams" (with T.M. Antonsen, Jr.), Physs.

Fluids B (1993).

"Theory of Gyro-Traveling-Wave Tubes at Cyclotron Harmonics" (with G.S. Nusinovich), Int. J. Electrom, 72 (5-6). p. 895 (1992).

"Theory of Relativistic Gyro-Twistron" (with G.S. Nusinovich), Phys. Fluids B, 4 (4), p. 10.58 (1992).

"Large-Signal Theory of Gyro-Traveling-Wave Tubes at Cyclotron Harmoncs" (with (j.S. Nusinovich), I..E.E.E. Tran. Plasma Sci, 20 (3), p. 170 (1992).

"Efficiency of Frequency Up-Shiffed Ciyrodevices: Cyclotron Harmones Versus CARMS" (with G.S. Nusinovich and P.E. Latham), presented on Infrared of Millimeter Wave Conference, (December 1992)

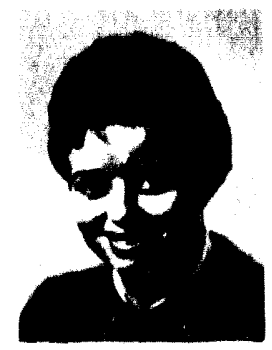

Mallka M. Meddahi

Staff Scientist

MS 8()-10)

(510) $486-5619$

meddahi (o) Ibl.gov

Joined 1.B1. in 1991

Ph.D., Physics, University of Paris 7, 1991.

Awards: Daniel Guignier Prize, 1991.

Research interests: nonlinear dynamics; accelerator studies; beam-beam effects; AI.S transverse damping scheme.

Selected publications: "Influence of a Wiggler Magnet in a Circular Machine" (with R. Schmidt), CERN-SL/ July 1992.

"Ideas for Future Synchrotron Light Sources" (with A. Jackson and W. Hassenzahl), EPAC 1992, Berlin. March 21-29, 1992.

"Proton-Antiproton Collisions at a linite Crossing Angle in the SPS" (with K. Cornelis and W. Herr), I.HC Nole 150(1991).

"Measurement of the Beam-Beam Effect as a Function of the Separation in L.E.P" (with K. Cornelis et al.), F:PAC 1990, Nice, June 11-16.

"Calculattons of the Tune Spreads Induced BeannBeam Eiffects in the Case of Partially Separated Beams" (with R. Schmidt), CERN-SI./90)-15(1990),

"Tracking Studies on the Be:am-Beam leffect in the CI:RN-SPS p-phar Collider" (with W. Herr and R. Schmidt), (T:RN-SI/YI-5(AP).

"Beam-Beam liffects in the Strong-Strong Regime at the CERN SPS" (with L. Evans, J. Gareyte and R. Schmidt). 1989 Part. Acc. Conf, Chicago, March 2023.

"Lxperimental Study of a Beam lixcitation in the Presence of the Beam-Beam Interaction" (with K. ('ornelis, R. Schmidt and D. Vandeplasiche), SPS/ AMS/Note ') (1). 


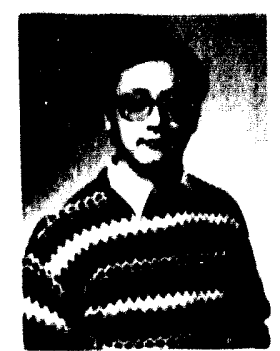

\section{Hiroshi Nishimura}

Stalf Scientist

MS 80 -101

(510) 486-576.3

hiroshi@lbl.gov

Joined L.BL in 1985

Ph.1)., Physics, University of Tokyo, 1982.

Research interests: accelerator physics for $\mathrm{AIS \text {: }}$ modeling and simulation code construction for real accelerator control using the novel programming methodologies like OOP.

Selected publications: "Dynamic Accelerator Modeling Uses Objects in Iiffel," (computers in Physics, 6. p. 456 (1942).

"Framework for Control System Development" (with C.W. Cork), to appear in the Pros: of the Intermotiomal Conference on Aceelerator and Large Physics Combrol Systems. Tsukuba, Japan (1991).

"Vertically Integrated Simulation Tools for Selt. Consistent Tracking and Analysis" (with I: Furest). Proc. Part. Acrel. Coml. (CH2669) p. 13(14 (1986)).

"Dynamic Aperture of the AIS Booster Synchrotrun." (with C. Kim). Pros. Part. Acrel. Conff. (112669, p) $132(1989)$.

"The Eiffects of Insertion Devices on the Behavior in the AI.S" (with A. Jackson. F. Forest and M.S.

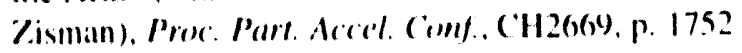
(1989)

"TRACY, A Tool for Accelerator Designn and Analysis." European Part. Accel Cont., p. $8(1) 3$ (1988)

"Particle Simulation Code for Non-Relativistic l:lectron Bunch in I.ASERTRON," Proc lincar Accel (iml. (iS)-84-11, p. 165(1984).

"LASERTRON: I aser Triggered RF" Source for linacs in the TeV Region" (with M. Yoshiokal el al.). Pros: lincar Arcel. Comf.. (iS1-84 11. p. 469)(1984).

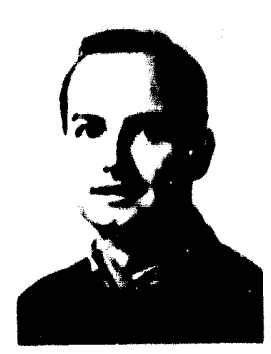

Rohert A. Rimmer

Staff Scientist

MS $71-250$

(5I()) $486 \cdots 624.3$

rimmer (ii) /hl.gov

Joined I. I3I in 1988

Ph.D.. IElectrical linginecring, Lancaster University. (IK. I)88, suhject: High Power Microwave Window Fiilures.

Research interests: computer simulation of high frepuency electromagnetic problems, Higher-Order. Mode suppression in RF cavities and structures. microwave windows, beam impedance of accelerator components.

Selected publications: "Rl: Cavity Development for the Pl:P.II B-Factory," Proce. Int. Workshop om B. Fartorie's, Bl.WS92, KEK, Japan, Nov, 17-20, 1992

"Higher ()rder Mode Damping Studies on the PlEP-II B-Factury RI" (avity" (R. Rimmer et al.), P'rof. 1902 Europ. lart. Accel. Comf. . Berlin. Germany, March 24 28. 1992 .

"An Rl: Cavity for the B-Factory" (R. Rimmer et al.).

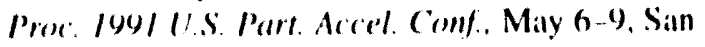
Francisco.

"Modes of lilliptical Waveguides; a Correction" (with D.A. (ioldherg and I.J. Laslett). IEEE: Trams. MTT.

Vol. 38, No. I1, pp, 16013-160) (November 1990).

"Beam Impedance Measurements on the Al.S Curved Sector Tank" (R.A. Rimmer el al.), Pros I log() Europ Part. Accel. Conl., June 12-16. Nice, lirance, 1.BI. $2 \times 192$.

"Determination of laailure Mechanisms of RF: Cavity Aperture Windows." Proc 1989 IEEE Part. Accel. (oinf.. March 20 23, Chicago.

"High Power Microwave Window lailures," Ph.D. Thesis, I.ancasler I niversity, UK, (available on microfilm from the university library) (October 1988). 


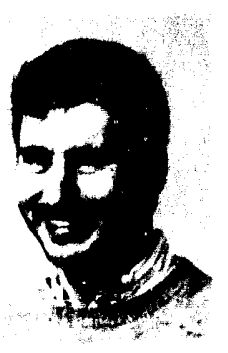

David S. Robin

Staff Scientist

MS B7IH

(5I0) 486-6028

robin@csa.lbl.gov

Joined LBL in 1991
Ph.D., Physics, University of California at Los Angeles, 1991.

Research interests: studies of the linear and non linear dynamics of lepton storage ring colliders.

Selected publications: "Quasi-Isochronous Ring Flavor Factories" (with C. Pellegrini), Rare and Exclusive B and K Decays and Novel riuvor Factories (1992).

"Isochronous Storage Rings and High-Luminosity Electron-Positron Colliders" (with C. Pellegrini), CP Violation and Beauty Factories and Related Issues in Physics, Annals of the New York Academy of Sciences, Vol. 619 (1991).

"Quasi-Isochronous Storage Ring" (with C. Pellegrini), Nuclear Instruments and Methods, A.30I, p. 27-36 (1991).

"Energy Density Enhancement in a Quasi-Isochronous Storage Ring" (with C. Pellegrini), Proceedings of the 1990) IEEE Particle Accelerator Conference (1990).

"Conceptual Design of a High Luminosity 5I0 MEV Collider" (with C. Pellegrini and M. Cornalcchia), Proceedings of the 1990 IEEE Particle Accelerator Conference (1990).

"A High Luminosity Superconduction Mini-Collider for Phi Meson Production" (with C. Pellegrini et al.). Proceedings of the 1990 IEEE Particle Accelerator Conference (1990).

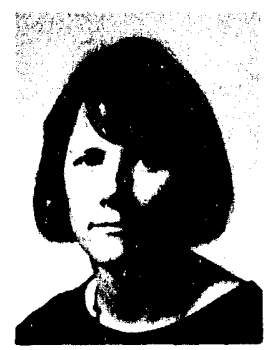

\section{Lindsay C. Schachinger}

Staff Scientist

MS 80-101

(510) 486-5009

lindsay@sesame.lbl.gov

Joined LBL in 1990

Ph.D., Physics, Rutgers University, 1978.

Research interests: accelerator simulation and modeling both for design and controls, non-linear dynamics, controls and modeling for accelerator physics studies in circular accelerators.

Selected publications: "Summary of the Working Group on Modeling and Simulation," Proceedings of the Advanced Beam Dynamics Workshop on Effects of Errors in Accelerators, Their Diagnosis and Corrections, AIP Conference Proceedings No. 255, Particles and Fields Series, 48, Corpus Christi, TX (1991)

"Experimental Investigation of Nonlinear Dynamics in the Fermilab Tevatron " (with A. Chao et al.), Physical Review Letters, Vol. 6I, p. 2752 (1988).

"Teapot: A Thin-Element Accelerator Program for Optics and Tracking" (with R. Talman), Part. Accel, 22. p. 35 (1987). 


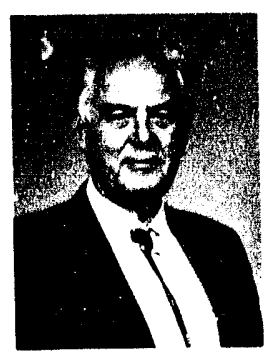

Frank Selph

Senior Scientist

MS B71H

(510) 486-6902

Fbselph@csa2.lbl.gov

Joined LBL in 1962
M.S., Physics, Univ. of California, Berkeley, 1963. Joined LBL in 1962, retired 1991. Continues to work on acceleratur projects.

Current research: ALS linac improvement, design of a storage ring for improved ion stripping.

Selected publications: "Magnetic Ring for Stripping Enhancement," LBL 82940 (Oct. 1992).

"Operating Experience with the ALS Linac" (with D. Massoletti), Proc. of the 1991 Part. Accel. Conf., IEEE 91CH3038-7, pp. 2978-80.

"Compensation of Beam Loading in the ALS Injector Linac," Proc. of the 1988 Linear Accel. Conf., CEBAF Report 89-()()1, pp. 58()-82.

"Wakefield Effects in the Two-Beam Accelerator" (with A. Sessler), NIM, A244, pp. 323-29 (1986).

"Acceleration of Uranium at the Bevalac" (with J. Alonso et al.), Science, 217, pp. 1135-37 (1982).

"The Next Generation of Relativistic Heavy Ion Accelerators" (with H. Grunder and Ch. Leeman), Proc. of the Symp. on Heavy Ion Research, G.S.I. Darmstadt, Germany (1978).

"The Status of the SuperHILAC" (with H. Grunder), Proc. of the 1976 Proton Linac Accel. Conf.. Chalk River AECL-5677, pp. 54-61 (1976).

"A Method for Obtaining Linac Beams of Continuously Variable Energy," Proc. of the 1970 Proton Linac Accel. Conf., Natl. Accel Lab., Batavia, pp. 868-879 (1970).

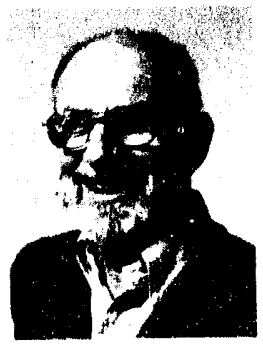

Andrew M. Sessler

Senior Scientist

Group Leader

Collider Physics Group

MS B7IH

(510) 486-4992

amsessler@LBL.

Joined LBL 1961

Director 1973-1980
Ph.D., Theoretical Physics, Columbia University, 1953.

Major awards: E.O. Lawrence Award by U.S. Atomic Energy Commission; U.S. Particle Accelerator School Prize; Leland J. Haworth Distinguished Scientist, Brookhaven National Laboratory; member, National Academy of Sciences.

Research interests: beams in plasmas; conventional and novel high energy accelerators; free-electron lasers.

Selected publications: "Transverse Resistive Wall Instability in the Two-Beam Accelerator" (with D.H. Whittum and V.K. Neil), Phys. Rev. A, 43 (1991).

"Relativistic Klystrons for High-Gradient Accelerators" (with G.W. Westenskow et al), Proc. of 1990 Linear Accel. Conf. (1991).

"Standing-Wave Free-Electron Laser Two-Beam Accelerator" (with D.H. Whittum, et al.), Nucl. Instr. \& Meth. in Phys. Res. A306 (1991).

"Radio-Frequency Beam Conditioner for Fast-Wave Free-Electron Generators of Coherent Radiation" (with D.H. Whittum and L.-H. Yu), Phys. Rev. Lett. 64 (1992).

"Photon Storage Cavities" (with K-J. Kim), Nucl. Instr. \& Meth. in Phys. Res. A318 (1992).

"Free Electron Laser Generation of VUV and X-Ray Radiation Using a Conditioned Beam and Ion-Channel Focusing" (with L.-H. Yu and D.H. Whittum), Nucl. Instr. \& Meth. in Phys. Res. A3/8 (1992). 




\section{Ferdinand Voelker}

Senior Scientist

MS 71-259

(510) 486-7237

ferd@lbl.gov

Joined LBL in 1952
M.S., Electrical Engineering, University of California, Berkeley, 1949.

Research interests: damping of HOM in RF cavities; study of multi-electrode kickers for particle beam; beam impedance measurements.

Selected publications: "ALS Longitudinal Kickers," presented at the 1992 Accelerator Instrumentation Workshop, LBL, Berkeley, CA, Oct. 27--30, 1992, LBL-33088.

"Higher Order Model Damping Studies on the PEP-II B-Factory RF Cavity" (with R. Rimmer, D. Goldberg, G. Lambertson, et al.), presented at the 3rd European Particle Accelerator Conference, Technical University of Berlin, Gerınany, March 24-28, 1992, LBL-32549.

"Calculations on RF Cavity Feedback Using Simple Analytic Model" (with Glen Lambertson), (1991).

"Technique for Beam Impedance Measurements Above Cutoff" (with G.R. Lambertson, A.F. Jacob, and R.A. Rimmer), presented at the European Particle Accelerator Conference, Nice, France, June 12-16, 1990, LBL-28190.

"A High-Frequency Schottky Detector for Use in the Tevatron" (with D.A. Goldberg, W. Barry, and G.R. Lambertson), presented at the Particle Accelerator Conference, Washington, D.C., March 16-19, 1987, I.BL -22273 .

"An Array of 1 to $2 \mathrm{GHz}$ Electrodes for Stochastic Cooling" (with T. Henderson and J. Johnson), presented at the 1983 Particle Accelerator Conference, Santa Fe, NM, March 21-23, 1983.

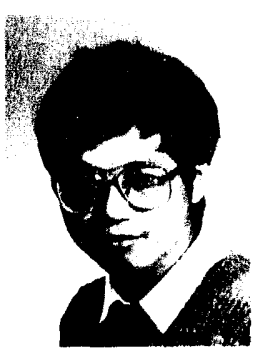

\author{
Changbiao Wang \\ Visiting Researcher \\ University of Electronic \\ Science and Technology \\ of China \\ MS B71H \\ (5I0) 486-6731 \\ cbwang@lbl.gov \\ Joined in August 1991
}

Ph.D., Electrophysics, University of Electronic Science and Technology of China, Chengdu, China, 1987.

Research interests: electron cyclotron resonance maser; free-electron lasers; electron beam conditioner; relativistic klystron simulation.

Selected publications: "Conditioner for a Helically Transported Electron Beam," to be published in the Proceedings of the Third Workshop on Advanced Accelerator Concepts (1992).

"The Problem of Stability of the Equilibrium Helical Orbit in Free-electron Lasers," Sinica Physica (1992).

"Comment on 'Gyrokinetics of Transverse-MagneticMode Gyrotron, Gyropeniotron, Cyclotron Autoresonance Maser and Non-wiggler Free-Electron Laser Amplifiers'," Phys. Fluids B3, p. 498 (1991).

"Comment on 'New Class of Unstable Orbits of the Equilibrium Electrons in Free-Electron Lasers'," Appl. Phys. Lett., 57, p. 837 (1990).

"Small-Signal Analysis of a Free-Electron Cyciotron Resonance Laser," Phys. Rev. A, 38, p. 215 (1988).

"Free-Electron Cyclotron Resonance Lasers," Appl. Phys. Lett., 53, p. 1911 (1988). 


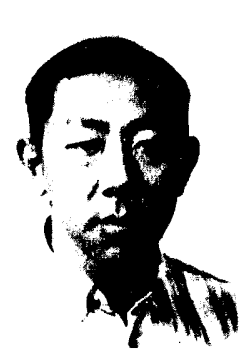

Ming Xie

Staff Scientist

MS B7IH

(510) 486-5616

mingxie@lbl.gov

Joined LBL in 1988

Ph.D., Physics, Stanford University, 1988.

Research interests: free electron lasers, optics, synchrotron radiation.

Selected publications: "Performance of Hole Coupling Resonator in the Presence of Asymmetric Modes and FEL Gain" (with K-J. Kim), Nucl. Instrum. Meth. , A318, p. 877, (1992)

"Three-Dimensional Theory of the Small-Signal HighGain Free Electron Laser Including Betatron Oscillations" (with Y. Chin and K-J. Kim), Physical Review A. vol.46, No.10, p. 6662, (1992).

"Self-Amplified Spontaneous Emission for Short Wavelength Coherent Radiation," (with K-J. Kim), $L B L$ report. No. 32288, (1992).

"Three-Dimensional Simulation of a Hole-Coupled FEL Oscillator," (with S. Krishnagopal, K-J. Kim, and A. Sessler), Nucl. Instrum. Meth. , A3/8, p. 661 . (1992).

"Hole Coupling Resonator for Free Electron Lasers" (with K-J. Kim), Nucl. Instrum. Meth. A 304, p.792, (1991).

"Stability and Performance of CDRL-FEL," (with K-J. Kim), Nucl. Instrum. Meth. A 304, p. 146, (1991).

"Eigenmode Analysis of Optical Guiding in Free Electron Lasers" (with D.A.G. Deacon and J.M.J. Madey), Physical Review A, vol. 4l, No. 3, p. 1662 , (1990).

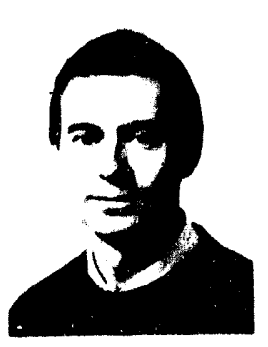

Alexander Zholents

Staff Scientist

MS B71H

(510) 486-7533

zholents@lbl.gov

Joined LBL in 1992

Ph.D., Experimental Physics, Institute of Nuclear Physics, Novosibirsk, Russia, 1983. 1983-1992: Scientist at INP, Novosibirsk.

Research interests: accelerators, dynamics of the charged particle beams, B-Factory.

Selected publications: "Beam-Beam Effects in Electron Storage Rings," Lecture Notes in Physics, v. 400 , p. 321 (1992).

"A Combined Symmetrical and Asymmetrical BFactory with Monochromatization" (with A. Dubrovin), IEEE, v. 5, p. 2835 (1991).

"Beam-Beam Effects with Large Dispersion at the Interaction Point" (with A. Gerasimov and D. Shatilov), NIM, A305, p. 25 (1991).

"Synchrotron Radiation Masking on Asymmetric $6.5 \times$ 4.3 - GeV B-Factory" (with V. Blinov and A. Matveev), IEEE, v. 4, p. 2342 (1991). 


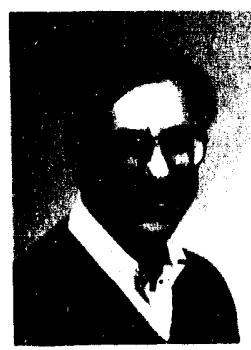

\section{Michael S. Zisman}

Senior Scientist

Group Leader

High Luminosity

Collider Physics Group, MS B71H

(510) 486-5765

zisman@lbl.gov

Joined LBL in 1966

Ph.D., University of California, Berkeley, 1972.

Research interests: design of electron storage rings and high-luminosity electron-positron colliders; beam instabilities; collective effects; design of PEP-II asymmetric B factory; study of high-luminosity collider design.

Selected publications: "Physics and Technology Challenges of B $\bar{B}$ Factories," Proc. of 1991 Part. Accel. Conf., San Francisco, CA, May 6-9, 1991, p. 1. Also in Lecture Notes in Physics, Vol. 400, ed. M. Dienes, M. Month, and S. Turner, Springer-Verlag, Berlin, 1992, p. 600.

"PEP-II: An Asymmetric B Factory Based on PE:P" (with A. Hutton, for the SLAC/LBL/LLNL B Factory Design Group), Proc: of 1991 Particle Accelerator Conference, San Francisco, CA, May 6-9, 1991, p. 64.

"PEP-II Asymmetric B Factory: R\&D Results." (with J. Dorfan, A. Hutton, and W. Barletta, for the PLP-II Design Group), Proc. of European Particle iccelerator Conference, Berlin, Germany, March 24-28, 1992.

"B Factory RF System Design Issues," Pioc: of Intl. Conf. on B Factories: The State of the Art in Accelerators, Detectors and Physic.;, Stanford, CA, April 6-10, 1992.

"ZAP and Its Application to the Optimization of Synchrotron Light Source Parameters," Part. Accel. 23 p. 289 (1988).

"ZAP User's Manual" (with S. Chattopadhyay and J. Bisognano), Lawrence Berkeley Laboratory Report No. LBL-21270), December, 1986, unpublished. 
Technical Support Staff 


\section{Warren Harnden}

Mech. Engineering Associate

MS 70A-336.3

(510) 486-61.51

Joined L.BL in 1964

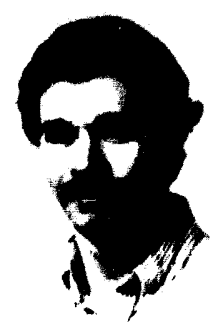

James Smithwick

Mech. Engineering Technologist MS 71-2.59

(510) 486-5227

Joined LBI in 1988
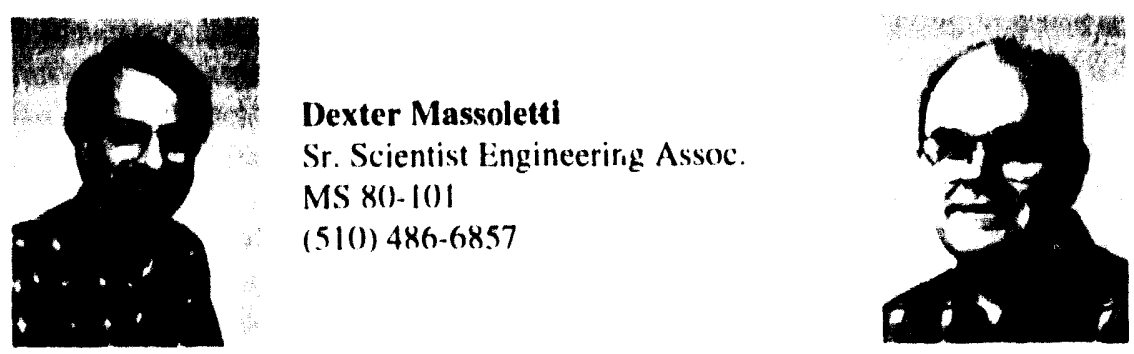

James Wise

Elect. Engineering Technologist MS 71-259

(510) 486-50)27

(51()) 486.6857

Joined LBL in 1962 


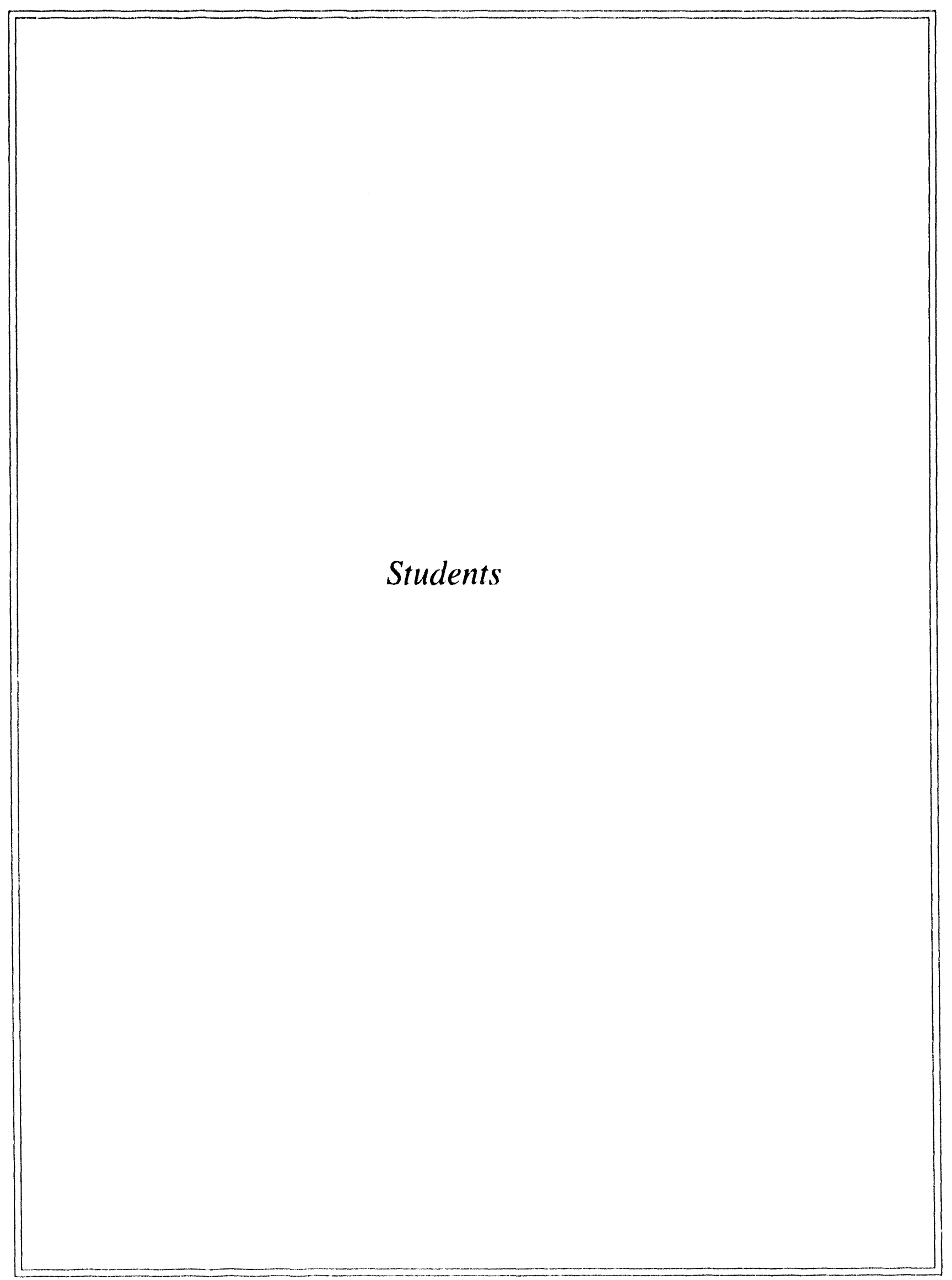




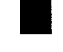

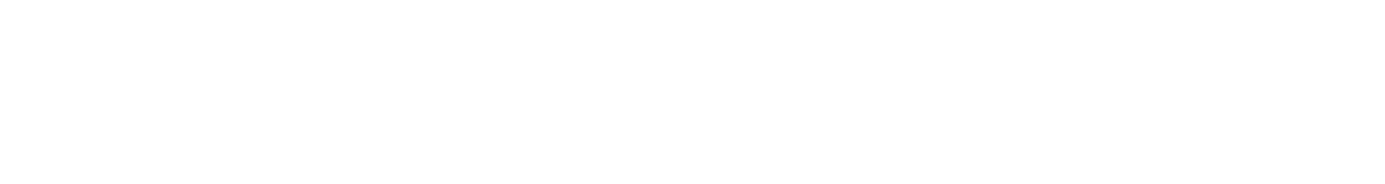




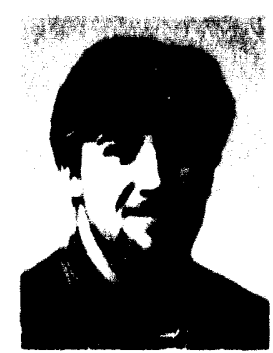

Jason Dunn

Graduate Student

UC. Davis

MS 71.259 (LBL)

(916) 752-150) (UCD)

UCDHEP::DUNN

Joined LBL in 1992

13.S., Physics, University of California at Davis, 1991

Research interests: accelerator physics, QCD at the HI:RA machine in Ciermany.

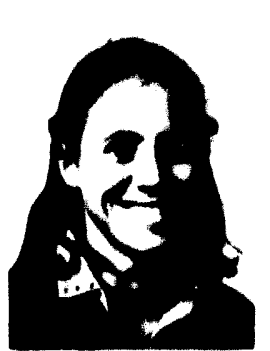

Giulla Fiorentinl

Ciraduate Student

MS B7IH

(510) 486-6.572

giulia (a) Ibl.gov

Joined L.BL in 1992

Ciraduate student at Universita' degli Studi di Milano, now at the I.awrence Berkeley I ab. as a Visiting Scholar for one year.

Research interests: study of beam dynamnics in relativistic klystrons and free electron lasers, computer modeling of heam transport and field dynamics in relativistic klystrons and SWFEL.

Selected publication: "Design of a Reacceleration I:xperiment Using the Choppertron," presented at SPIE Symposium (January 1993). 


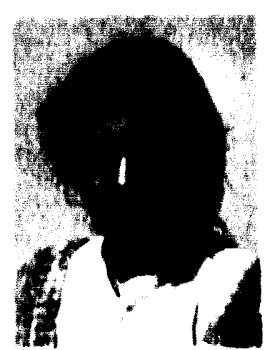

\section{Richa Govill}

(iraduate Student

UC. Berkeley

MS B BIH

(510) $4 \times 6.4973$

R. Govil(al.BI.

Joined I.BI in 1991

A.B., Univessity of California at Berkeley. Physics.

Major awards: Certificalte of Distinction. University Medal, UC, Berkeley.

Research interests: free-electron lasers; high energy beams in accelerators; semi-conductor lasers.

Selected publications: "Macroparticle Theory of a Standing-Wave lirec-lilectron Iaser Two-Beam Accelerator" (with K. Takayama and A.M. Sessler), Nucl. Instr. and Meth. in Phys. Re's., A.320 p. 587 (1992).

"Design of Rl' Conditioner Cavities" (with R.A. Rimmer. A.M. Sessler and H.(i. Kirk). Pros. Ith IIII. FEl. Comf. Kobe, Japan, August 23-28. 1992

"A Proposed Experiment for Beam Conditioning" ( with 1. Ben-7, i. 1.-H Yu and A.M. Sessler), Pros I Aih IIIt

FEL Comt. Kobe, Japan. August 23-28. 1992

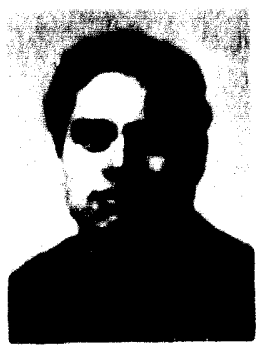

Kenneth LaMon

(iraduate Sludent

IC: Berkeley

MS B $71 \mathrm{H}$

(.510) 486.5756

Joined I. BI in I98X

A.B., U.C.. Berkeley, 1989. Student rescarcher HII:AR group 1988-89, CBP 1989. Presently (iraduate Student U.C. Berkeley; (iraduate Student Research Assistant with CBP since 1991

Research interests: theoretical heam physics. Ph.D. thesis topic: Particle and Radiati on Dynamics of Isochronous Storage Ring liree lilectron lasems.

Selected publications: "Removal of Singularities from Taylor Series," I.B3I. Report No. 2\%/689, IESCi Nole-77. August 1989.

"I ie Series in an Exlended Region of Phase Space." Jomrnal of Phisics A: Marhematical and Cieneral. 23. p. $3875(190(1))$. 


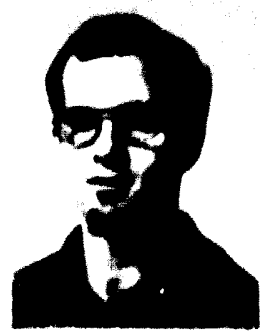

Steve Lidia

Reseirch Assuciate

MS $1371 \mathrm{H}$

(.51(1) $4 \times 6(-.5756$

Joined I B BI. in $\mid 901$
B.S. Iingineering Physics, University of Californial. Berkeley. 1941

Research interests: varriable polarization inscrtion devices; partially coherent, high intensity radiation sources: modelling and simulation of magnetic fiedd and synchrotron radiation sources.

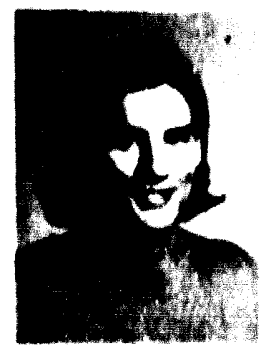

\section{Eric Wullace}

Student Assistam!

MS 71.259

(51) 4860.6553

Joined 1.131 , in 1992

Senior, University of California. Berkeley

Research interests: laser physics and optics

Selected publications: "I:xperimental Test of Hole (oupled lila. Rewnator Devigns llsing a ('W-HeNe l.ancr" (with W. P. Lecmans, M. Xic, K-J. Kim). presented at the SPll: Internattional Symposiat on laser lingincering. I.us Angeles, ('A. Jannuary 16.23. 1003. 1.31. Report No. 3.36(1),3

"I:xperimental and Simulation of Hole-Coupled Resonatter Modes with a (W HeNe Laser" (with W P. lecomans, M Xie. J.A Ialigholfer. K.J. Kim, and S (hattepadthyaly), presented at the 1 d th International IIII. Conference, Kuhe, Japan. August 23-18, 1992. 1.BI. Repurt No. $322 \times 5$ 
Administrative Support Staff 


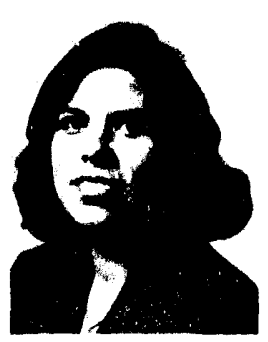

Martha Condon

Administrative Staff

Center for Beam Physics

MS 71-259

(510) 486-5792

CBP@lbl.gov

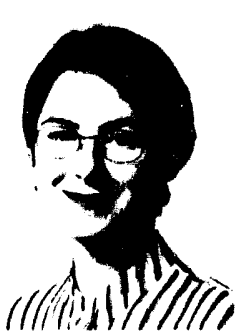

M. Mara-Ann

Administrative Staff

Center for Beam Physics

MS 71-259

(510) 486-5792

CBP@lbl.gov

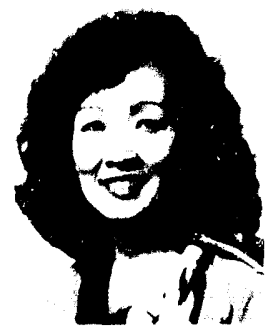

Joy Kono

Program Administrator

Center for Beam Physics

MS 71-259

(510) $486-6375$

joy (ni) bl.gov

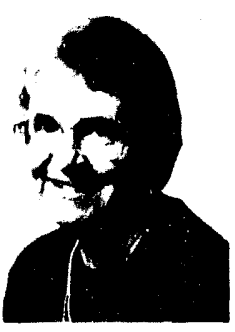

\section{Darlene Moretti}

Administrative Staff

Collider Physics Group

Center for Beam Physics

MS B71H

(510) $486-4182$

dmoretti@lbl.gov 
$\frac{2}{\frac{\pi}{8}}$ 


\section{CENTER FOR BEAM PHYSICS}

\section{Afriliates}

Femtosecond X-ray Source Collahoration:

R. Schoenlein, LBL

S. Dexheimer. LBI

C. Shank. LBL

PEP-Il B-Factory Collaboration

D. Hitlin, Caltech

P. Oddone. LBL

J. Dorfan. SLAC

M. Calderon, LLNL

SCRF/FEL Diagnostics Collaboration:

A. Schwettman, Stanford

T. Smith, Stanford

I. Ben-Zvi, BNL

S. Fornaca, TRW

FEL/SCRF Collaboration:

S. Benson, CEBAF

F. Dylla, CEBAF

C. Lecman, CEBAF

G. Neil, CEBAF

J. Bisognano, CEBAF

Photocathode/SCRF Collaboration

Chia-Ern Chen, Peking University

Two Beam Accelerator Collaboration:

G. Westenskow, LLNL

J. Haimson, Haimson Research Associates

Beam Test Facility:

J. Krupnik, LBL

C. Matuk, LBL

J. Hinkson, L.BL

G. Behrsing, LBI.

ALS Studies - B. Kincaid, LBL

CXRO Studies - D. Altwood. LBL.

Isospin Laboratory Studies - M. Nitschke, LBL

Migma-Based Accelerators - A. Ghiorso, LBL

Optical Coherence Studies - R. Littlejohn. LBL

Photocathode and Plasma Lens Studies - K. Leung. LBL

Short Wavelength FEL Collahoration - H. Winick. SSRL. Stanford 


\section{Publications}

(1991-1993) 


\section{CFNTEK FOR BEAM PHYSICS}

\section{Bublications - Externul Notes}

K.J Kim. "Spectral Bandwidh in Free Liectron laver

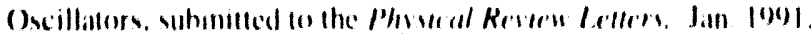
1.131. 3(1)192

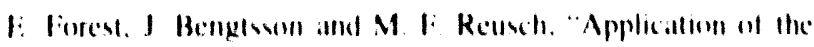

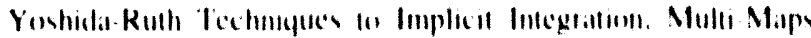

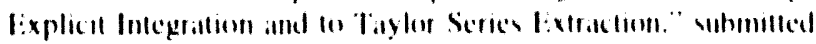

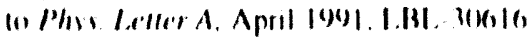

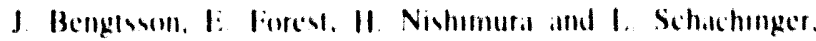

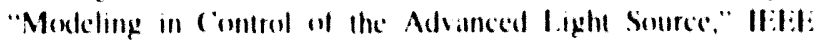

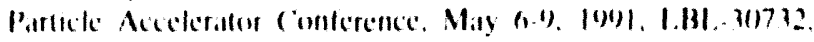
ISAP' 117

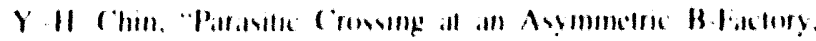

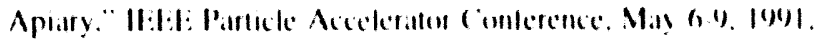
1.131. $-3(1) 701$

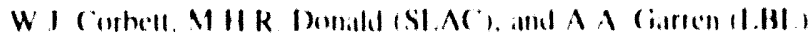

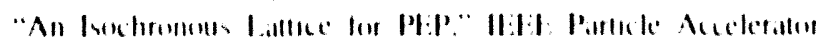

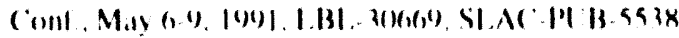

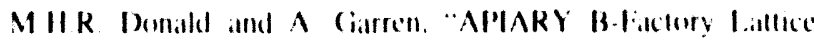

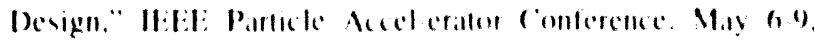
(1)9) I. B . $1(1)(1) \mathrm{x}$

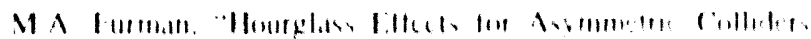

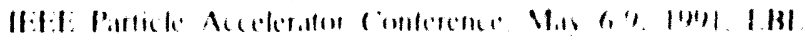
$3(1) \times 3$

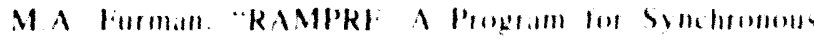

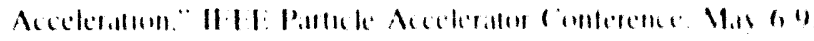
1001. 1.131. 301812.585 1. 171

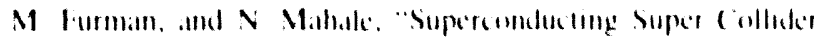

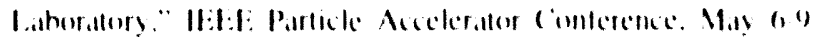

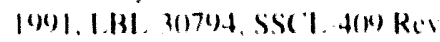

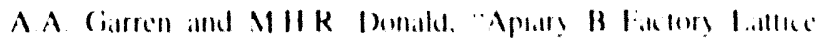

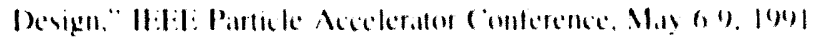
I.BI- $-306(1,5$

A A Garren, and M Sullivan. "APARY B Fanders Sepandmen

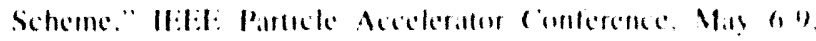
(0)1. 1.131. - 3(1)731)

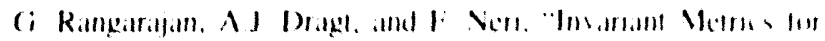

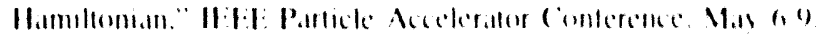
$|(0)|,|| 3 \mid,$,

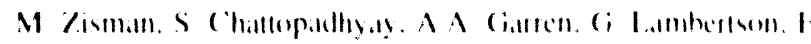

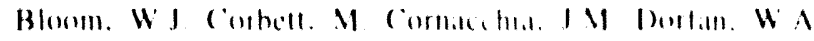

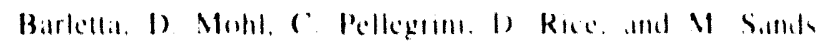

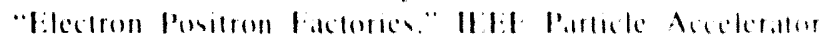

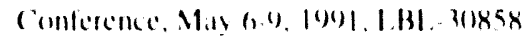

M Kivman, A Huflon, "Pl:P-II An Asymmetric B Factury

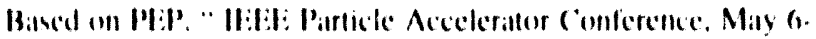
1). $\mid(1) 1,1,131,3(1857$

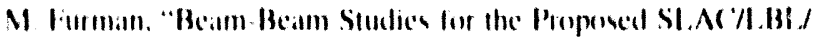

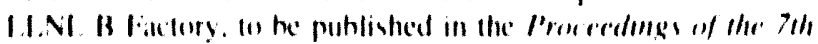

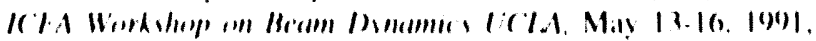
I.BI. 3(M)

II Viuman, "Repent of the Cirmup on Beam Beam liflect in circulat colliders." to he published in the Prosededeng of the

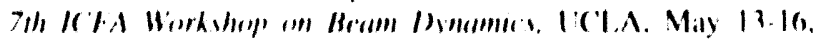
|U)।, I.131. 31015

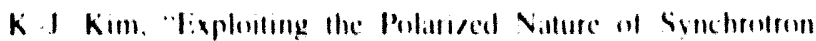

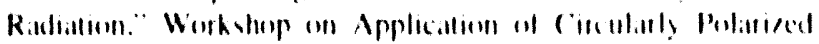

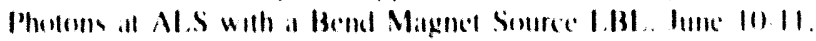
|w) I. IST i.143

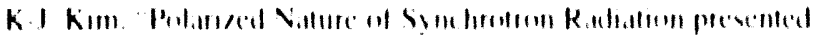

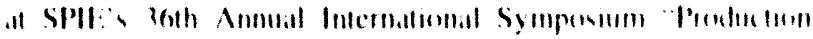

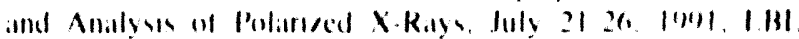
31242

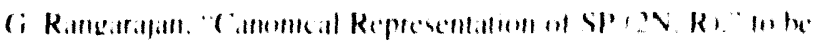

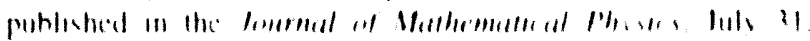
(w) I III 1110

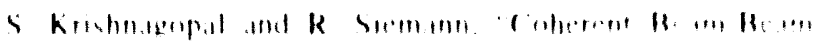

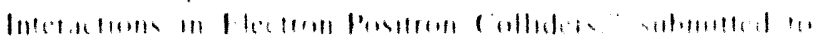

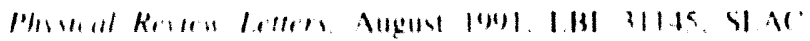
II 18 \&, 11

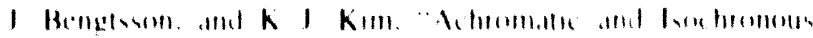

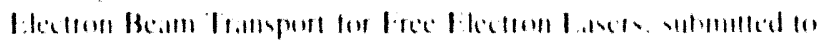

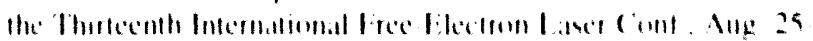

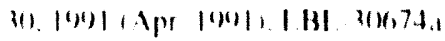



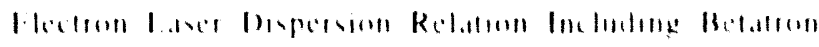

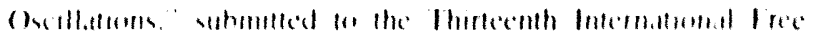
Ifetron I and (

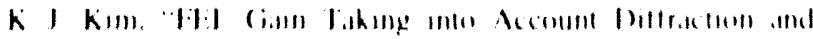

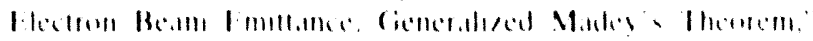

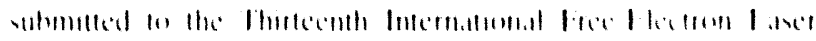

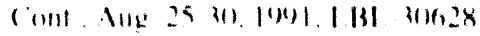

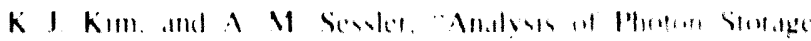

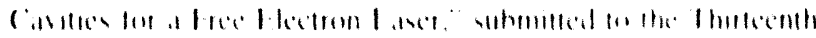

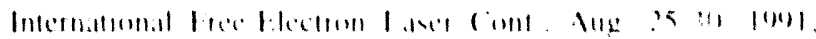
1.131. $316,27.1$

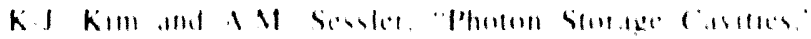

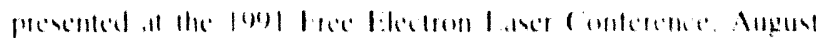
2930.1411 .131 .11119 


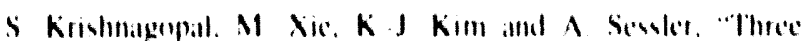



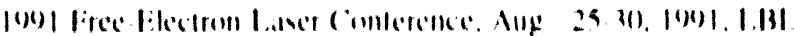
$311 \%$



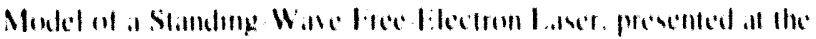

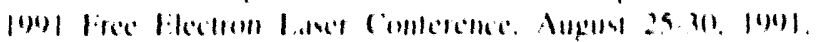
1.131. 31107

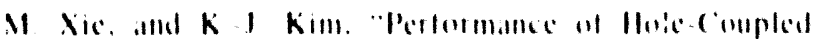

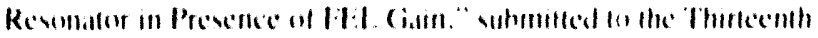

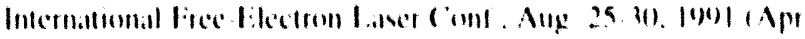
|W01). 1.131. 316,72.1

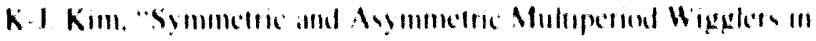

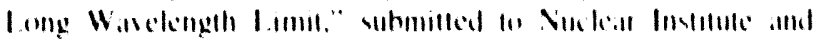
Methosh. September 1001, 1.131. 11200

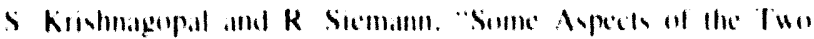

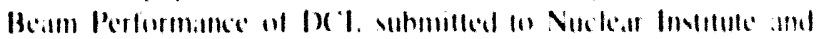

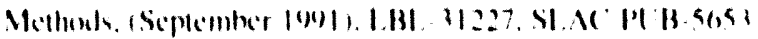

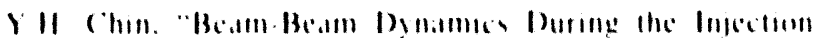

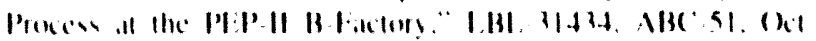
|था

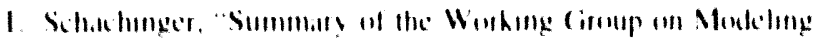

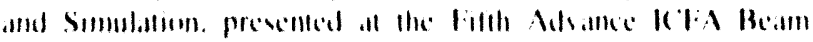

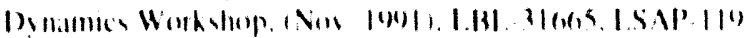

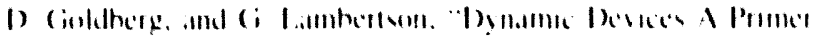

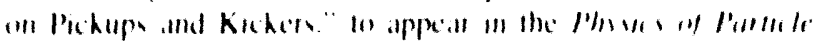

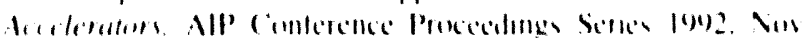

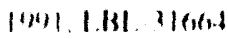

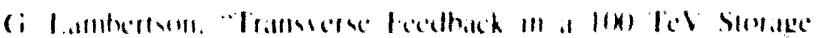

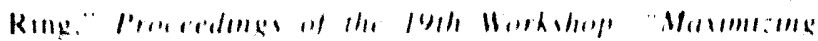

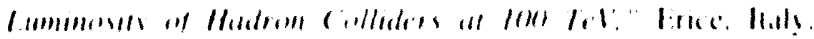

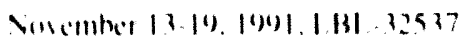

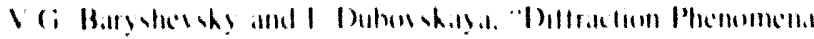

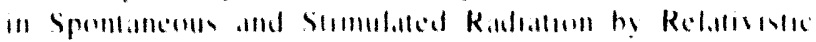

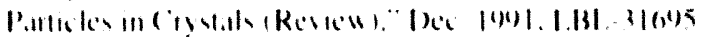

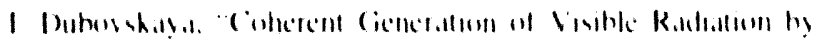

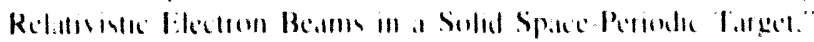
Dec $1001,1.131 .31(1) 4$

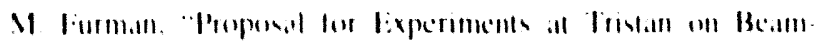

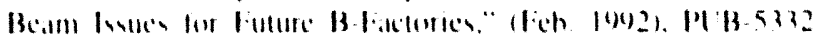
InI ARC $\cdot(1)$

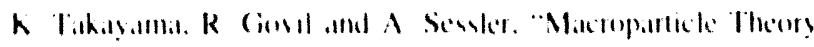

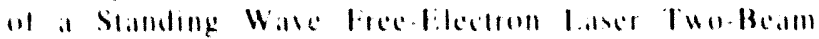

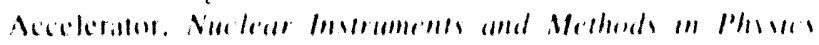

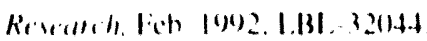

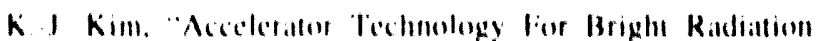
Heame." ins lled paper presented at I a Jollat lenternationna' Scheol

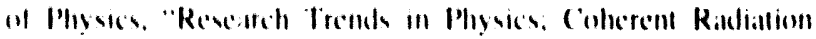

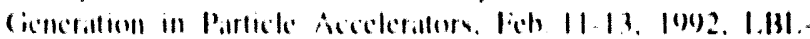
1111.5

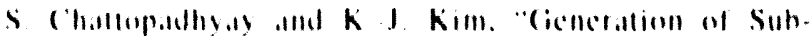

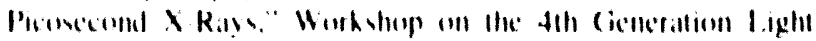

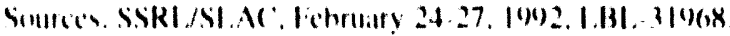

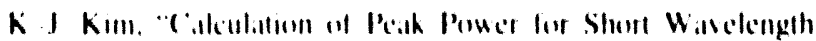

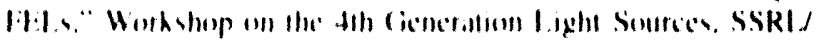

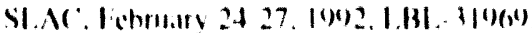

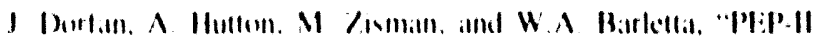

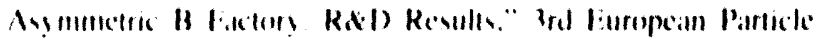

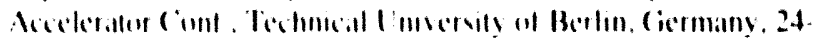

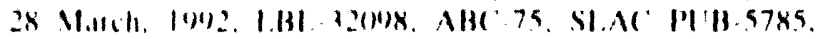
I.INI I I RI IC $11112 \times 7$

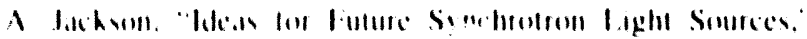

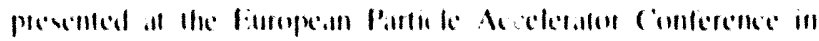

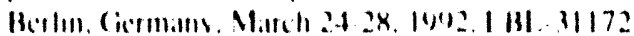

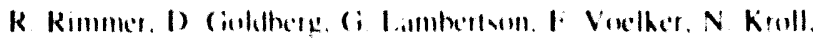

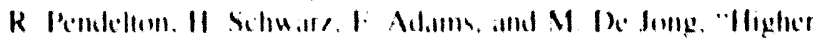

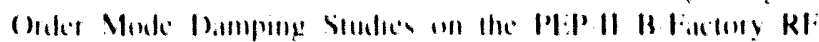

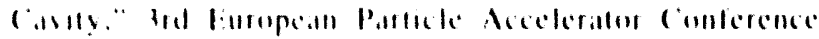

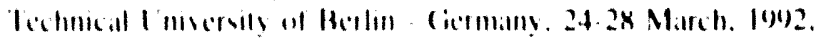
I.131. 3254". alld N18C 74

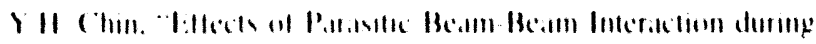

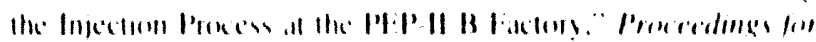

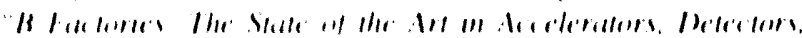

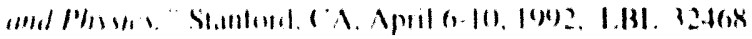

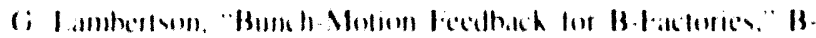

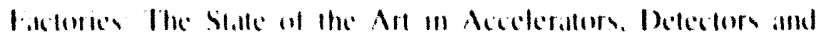

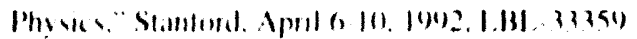

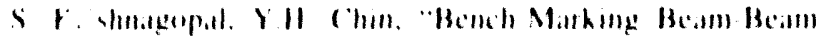

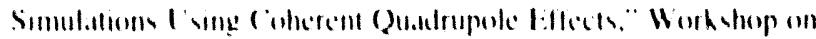

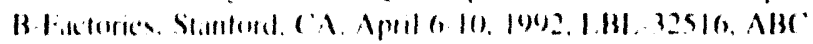
73

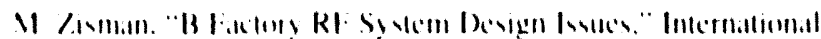

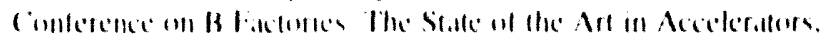

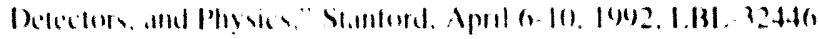
.Ind $N 136 \div 72$

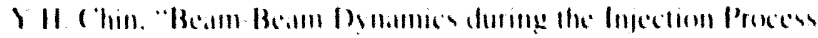

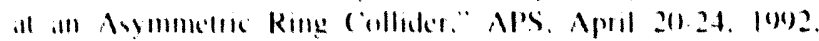
1.111. 31794.1

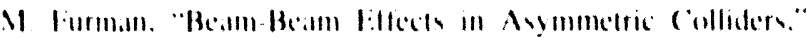
Als. April 211.24. 1002.1.131. 31792.1 
M. Lurman, "Beam-Beam Lowes in Asymmetric Colliders," B. Factorics: The State of the $A \mathrm{rl}$ in Accelerallors. Detectors and Physics." Stanford, April 6. 10, 1902, and alon the Als. Washinglon. April 20.24, 19012.1.131. 12561, and A136:.77

S. Krishnagupal. "Coherent Beam-Beam Simulantoms." Als. April 201 24. 1002.1.131.-31747a1.

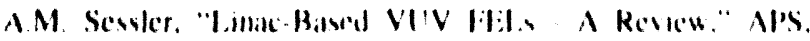
April 2(1)-24. 1902, 1.131,-31696)

Y.II. (hin. K.J. Kim and M Xic. "Thres-Dimemsiomal free Electron lasser Theory Including Betalron Oscillattoms."

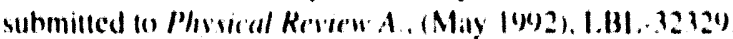

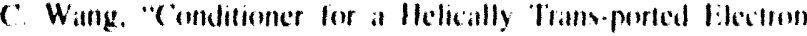
Bciam," May |W02, I.B1. 32222

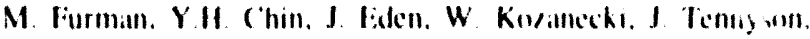

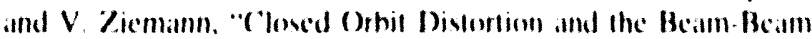

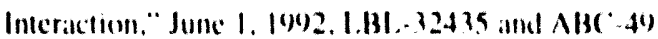

(i. Rangarajan and $A$ Seroler. "Semstionty Studies of at Standing Wave fire-lilectron Laser." Workshen on Ad anded Acceleratur concepts, Pl Jefferson. NY. Jume H.20. 1002. 1. B1. 32463

1. Wurtele, I) II. Whillum, and A M Server. "Impedinne Based

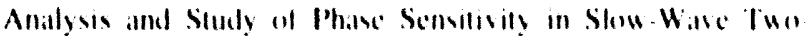

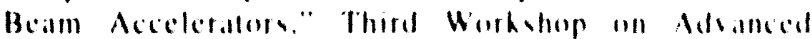

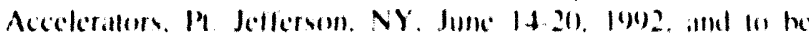

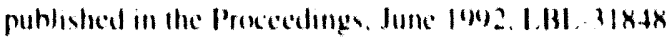

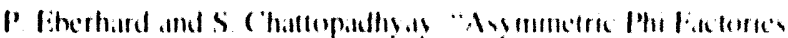

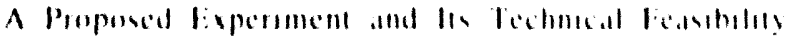

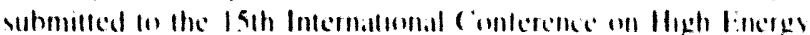
Accelerallors, July 211.24. 1042.1131.32291

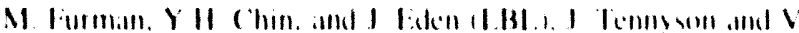

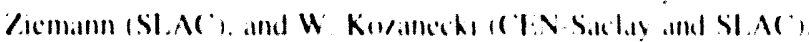

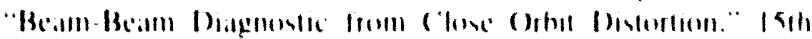

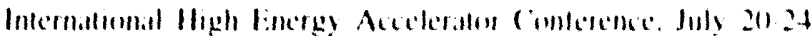

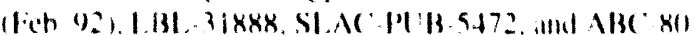

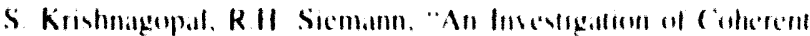

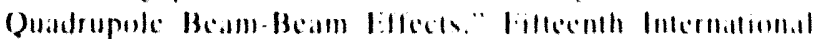

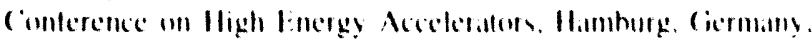
July $20.24,1902.1 .131,32581$

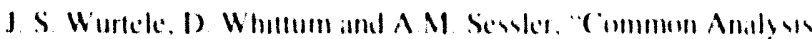
of the Relatesiste Klyutron and the Standing Wase fire

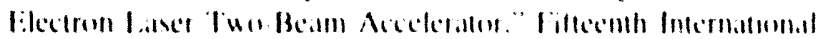

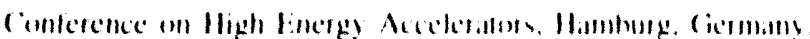
July $2(1) \cdot 24,1942,1.131 .325 \times 1)$

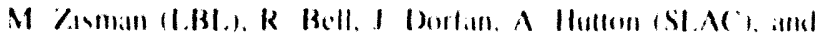

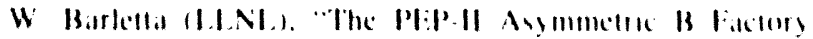

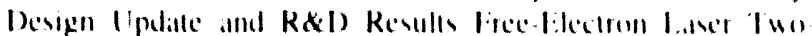

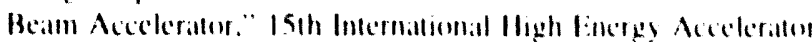

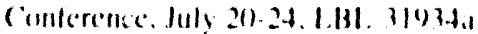

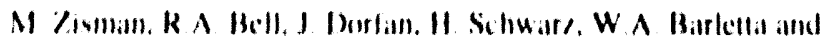

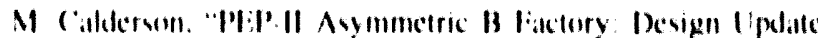
and ReD Resulds," I sth International confertence on lligh lencrgy Accelcraters dlll:A(')

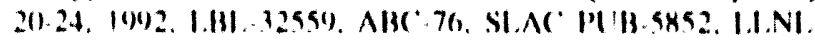
II RI J $1113 \times 11$

W Harlellet. A sewler and 1. II Yu. "Phwsially Trimspallent

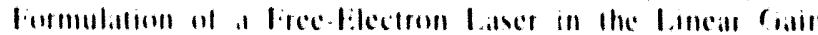

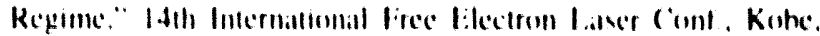

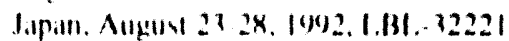

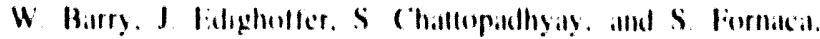
"lield Protile and lobsling Measurements on Higher Order

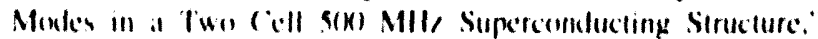

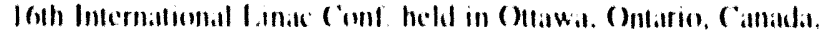
Aug 21.2x. 1002,1.131. $1217 x$

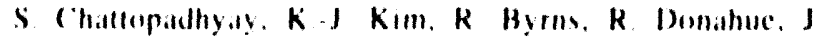

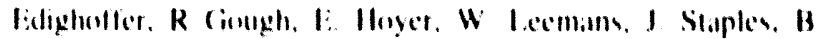

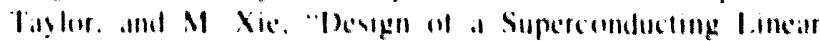

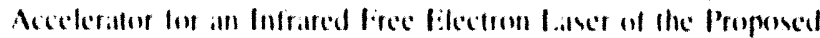

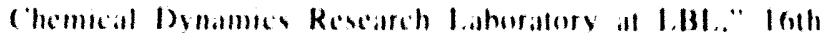

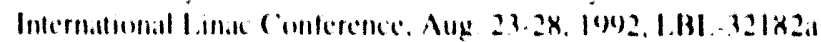

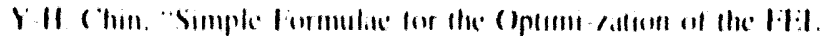

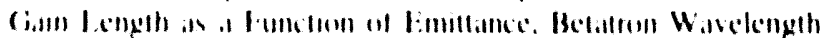

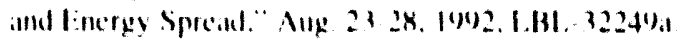

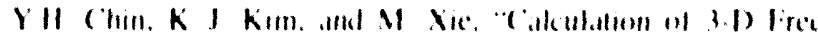

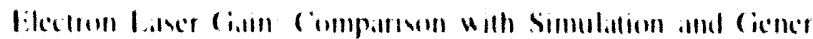

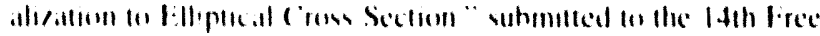

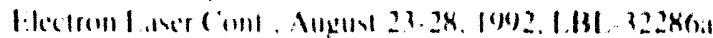

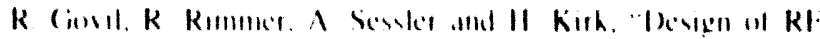

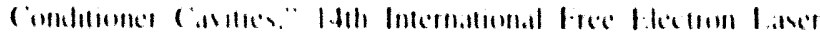

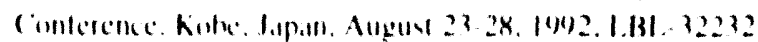

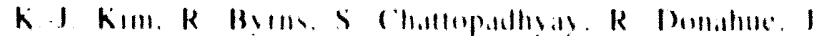

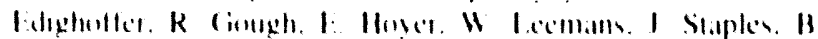

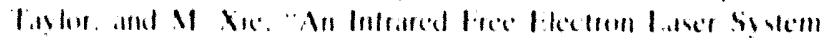

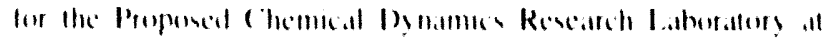

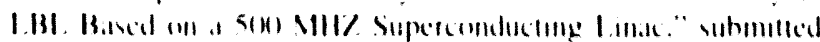

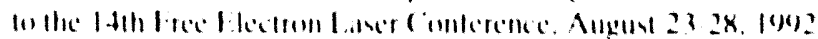
1.1.1. $122 \times 7.1$

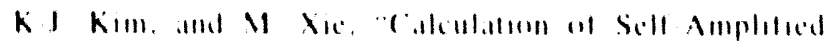

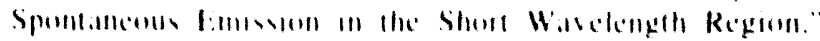

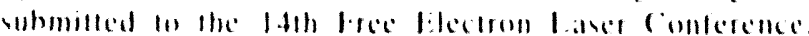
Aupul $212 x .1012 .1$.131. $322 \times x .1$

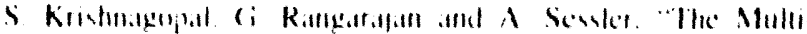

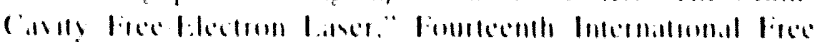

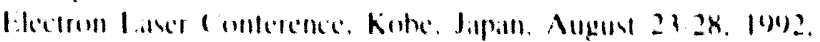
1.131. 12220

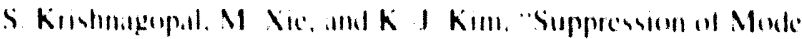

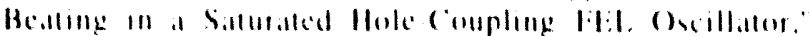

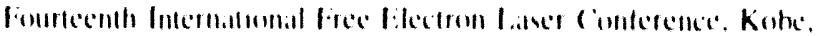

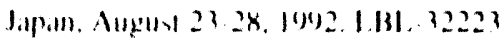


W Lecmams, I ladigholler. K - I Kinn and S Chollupinllyaly.

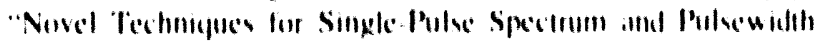

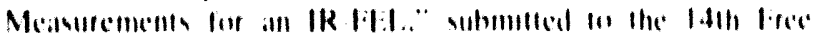

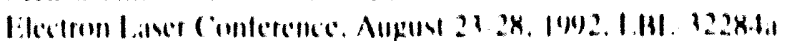

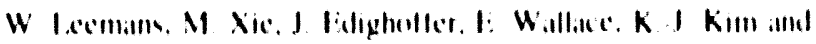

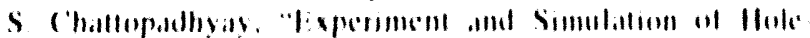

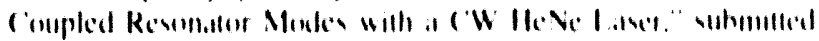

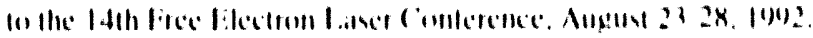
1.131. 32285

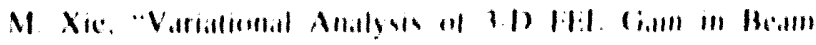

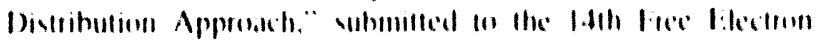

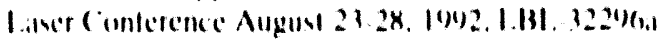

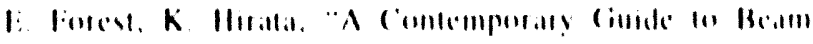

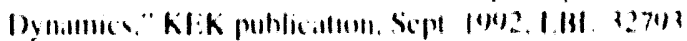

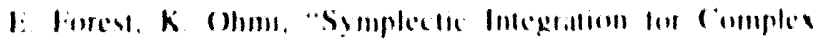
Wigglers." Klik publicallum, Siph 1012.1.131. 1270?

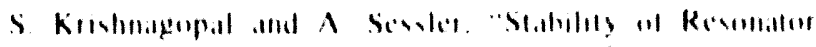

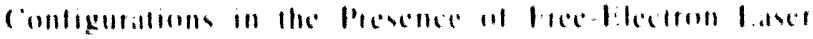

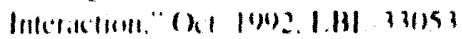

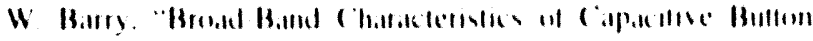

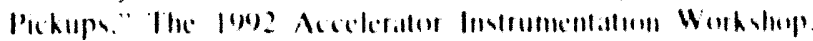

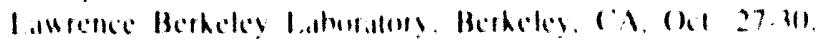
101) I. I.131. 323 34.,

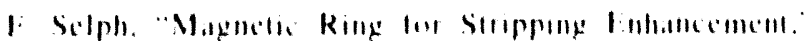

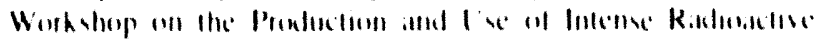

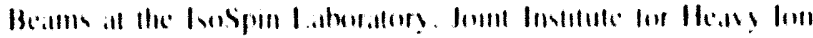

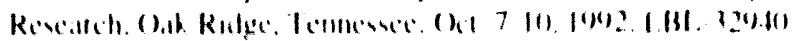

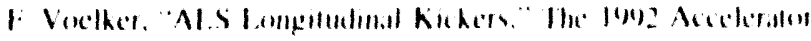

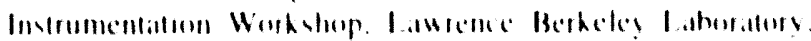

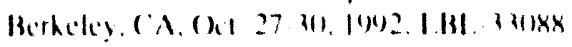

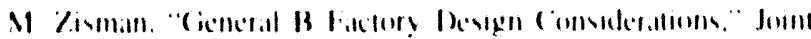

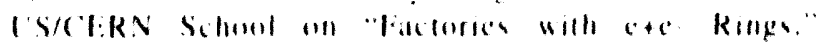

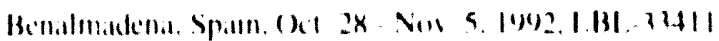

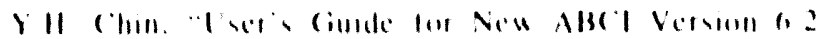

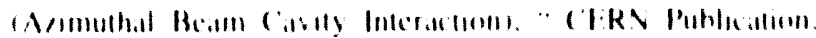

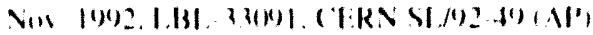

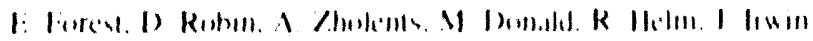

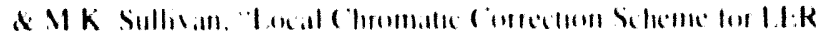

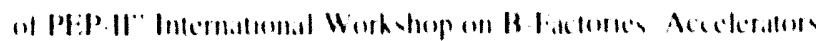

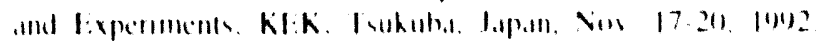
1.131. $333.47 \%$

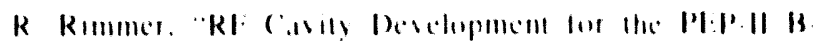

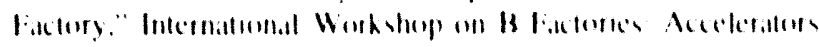

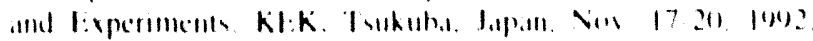
1.131 .333611

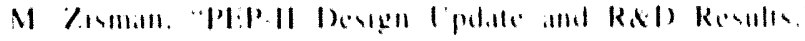

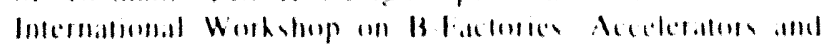

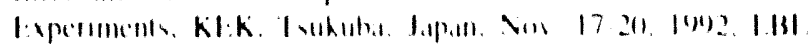
33.117

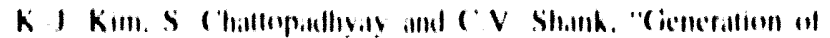

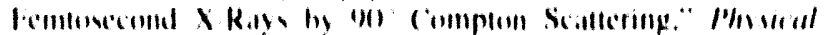



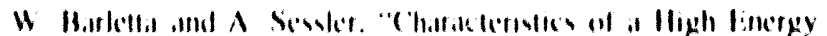

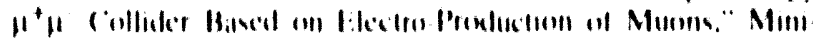

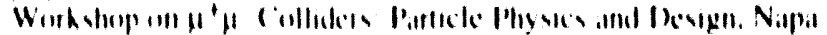

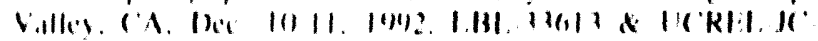
II (1)il

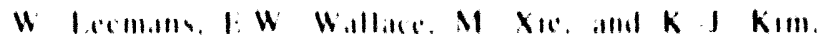

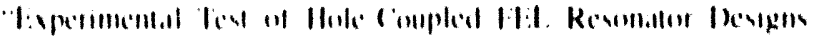

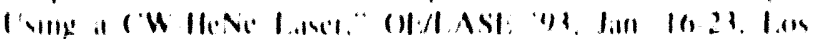
Angles. I,BI $13(6) 1$

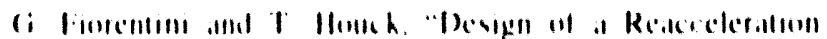

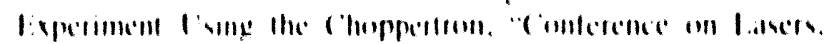

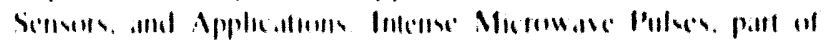

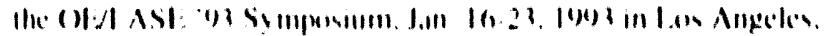
1.111. $124 \times 21$

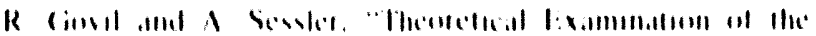

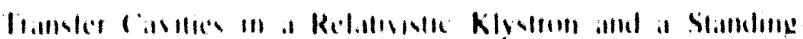

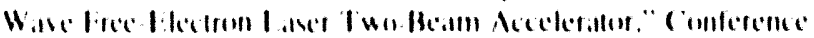

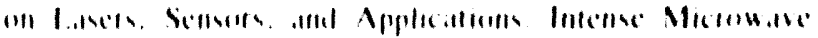

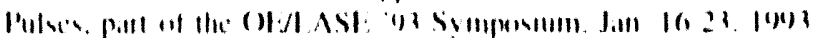

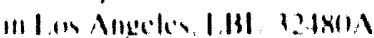

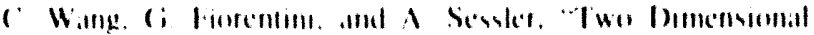

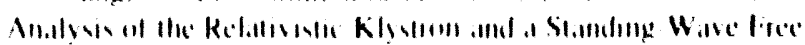

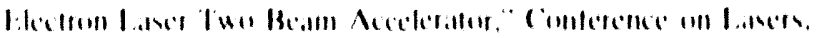

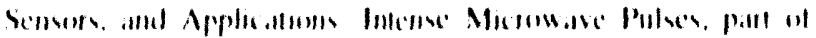

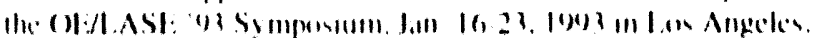
I.|31. $124 \times 1 \mathrm{~A}$

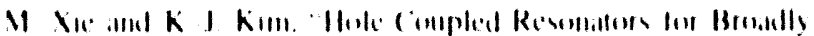

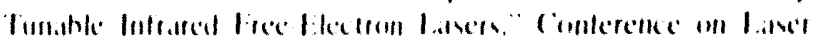

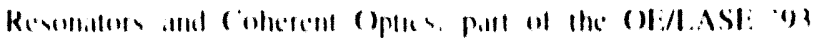

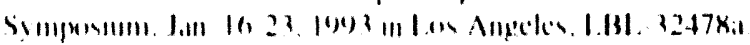

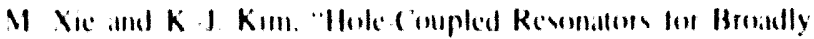

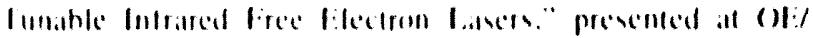

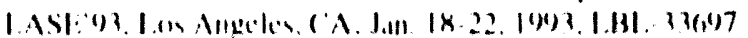

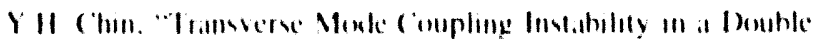

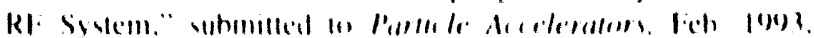

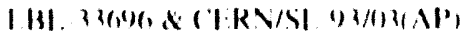

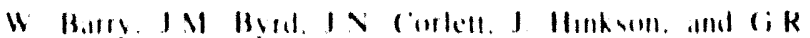

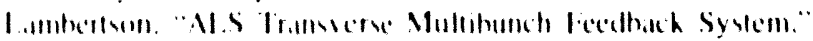

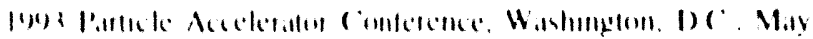

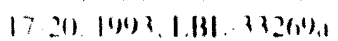

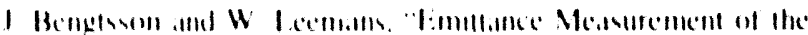

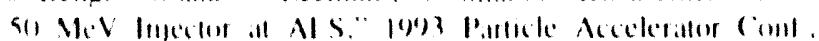

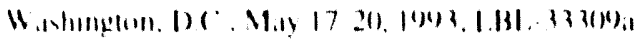

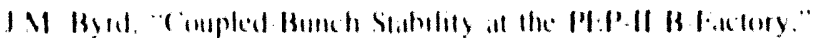

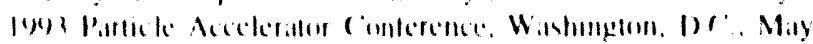
17211.1009 .1131 .1323 
JM. Byrd. "Simulation of the ALS Longetudinal Feedhack System," 1003 Particle Accelerator conlerence, Washinglon. D). . May 17.20, 1003, 1.B1. 33271.1

JM. Byrd. JN Corkll, "Coupled Bunch Stability at the ALS." 1903 Particle Accelerater (onference. Washington, D) May $17.20,1003,1,131,-3.270 \%$

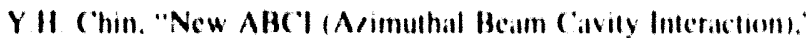
190) Particle Accelerator Conference, Washingenn, D) (.. May $17.20 .1093,1.131 .33268 \mathrm{a}$

M Conde, S Chatlopathyaly, K.J.Kim. K.N I cung, and $\mathrm{A} T$ Young. "I:lectron Quantum Yicles Irom a Barrum Pholek:alhode Illummited with Polaried lights." 1093 Particle Acceletator

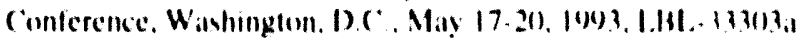

J. Corlell and s Byrd. "Mcasurements of the lligher ()rder

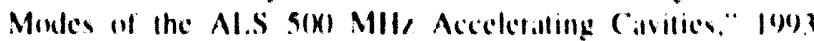
Particle Accelerattor Conference, Washington. D( . Mary 17.20. 199.1. I.BI.-13278:1

1. Corlell and R. Rimmer. "Impedance Measurements al components for the Als." I9y Particle Acecteralor conference, Wishinglon. D( . May 17.20. 1093. 1.131. 1327\%,

M. Donald, R Ilelm. J Irwin, It Fores, D) Rohin. A Tholems. and $\mathbf{M}$ Sullivan. "I.ocaliec (hromaticily Correction of l.on Beta Insertions in Sturage Rings," 1003 Particle Accelerator

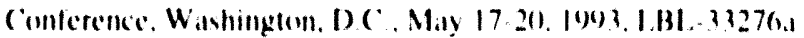

M.A Furman, J R Eden, "Beam-Beam Efflect lor the Ilip. II B Factury." lows Particle Acceleritor conlerence. Wahington.

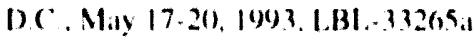

M. A Iurman. J R Lden, S. Krishnitgopal. "A Detanked Siudy on the Coherent Modes for Round Beams." log 3 Parlick Accelerator Conference. Washington. D) . Maly 17.20. 1903. I.BI. 33266.1

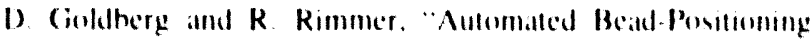

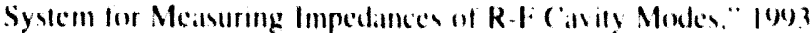
Particle Accelerator Conference. Washington. 1) (1) May 17.20. $1093.1 .31,-3.3290$ i

T Houck and (" Wang. "Dosign Siudy of a Microwane Driver for a Relativistic Klystron Two.Bcim Aceclerathr." /903 Particle Accelerator ('onference, Wishington, i) (". May 17.201 1993. 1.B1. $.3325 \times$ i
J S. Kim and A M Sensler. "Beam Sensilivity In Cavily Geometry in a Standing Wave Fil./TBA." I00? Particle Acceletillor (onferente. Washinglon. D). May 17.20, 1901. 1.111. 31257 .

Is Kim. A 11 Sewler. D. Whillum, and 't Houck, "Beam Break le in a Standing Wave lilal.TBA." 1093 Parlicle

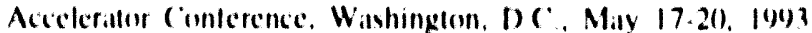
1.131. .32559

W Lecmatrs, (i Hehrsing, S (hatlopathyaly, K J Kim, J Krupnick. (C Malluk. IF Selph, and I. Stout. "Design of the Advanced Acederator Ten Beam l.ine at 1.131.." 1993 Particle Accelerator (onference. Washinglon. D). May 17-20, 1943. 1.131. $.3 .3118 \mathrm{a}$

M Meddalie and I Bengleson, "Sindy of Tramserse Coupled Bunch Instathlities by I sing Non-linear Tilylor Maps for the Advanced light Source (AL.S)," 1993 Particle Accelerator

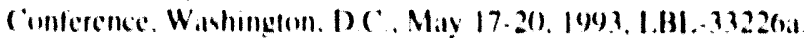

It Meddahi, J Bengesonen. K Keller. "Study of a Possible Energy I perate for the ALS and Medeling of the "Real lattice" hy a Nentelencar Taylor Series Map." 1903 Particle Accelerator (onference, Wishonglon. I) ('. May 17.20, 1093, 1.131.33225:

(i Portmann, I Benglssom. "The Closed-Lowp Photon Beam Stcering Algorithm for the Advanced light Source." /90? Particle Acederator Conterence. Accelerator Science and

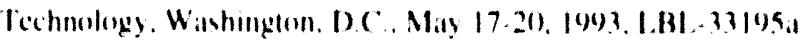

R. Rimmer, D) (inldherg. "Measurements of Higher.(O)der

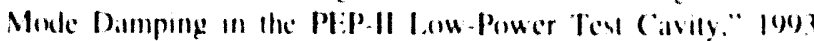
Pisticle Accelerater Conference, Washinglon. D) ( . May 17.20. 1903.1.131. 33312 i

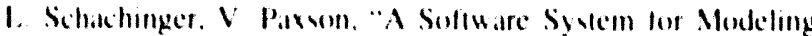
and controlling Accelerator Physics Parameders at the Advanced light Source." 1903 Particle Accelerator conference.

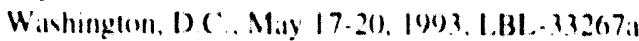

C II Wang. A Senter. "Two Demensional Simulations of the Relativistic Klystron and a Standing-Wate Free-bilectron laser


Wawhington. 1)(". May 17.20, 1903, 1.131,-33256a

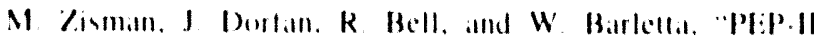
Aymmetric B fictory. Design ( pdate and REI) Resulde," I0) Parlicle Accelerator ('onference, Washinglon. D). ('. May 17.20. 1093.1 .131 .33311 i. 


\section{( WNTER FOR BEAM PIIYSIC'S}

\section{Publications - Internal Notcs}

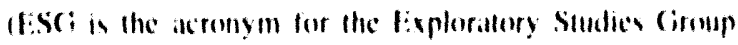

the mance used hy the ciroup prien lo hesoming the center for Beom

Physico,

11 Iurman "The Hourglass Reduction Factor for Asymmetric

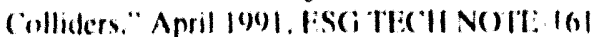

Various Authors, Vugraph Presentations, Intermational Workshop on Acceleraltors lor Asymmetric B-Fictories, (Ocluber 4.6. 1000, ESC TTECH NOTL:-162.

M. Furman. "Luminosity Formulas for Asymmetric Colliders with Beam Symmetries," Fehruary IOOI. ISS TICH NOTl:163.

W. Joho, "Fine Tuning of an Undulator with Edge Angled Pole Peces," January 25. 1001. ESG TICOI NOTL-164.

W. Joho, "Some Comments ahout the Harmonic Number $h=$ 328 for the Al.S Storage Ring," Jamuary 30, IO) I. IS( TISCH NOTE-165

M. Zisman. "Responses to B Factury Design Review Comments." March 10. 1901. LS( ; TEC H NOTE-166.

M. Furman and P. Mormon. "The Laskell Tune Shifl lor the B.

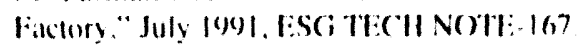

1. Selph, "Al.S Experimental l.ine," April 30, I94)I. ES(; TI: "II NOTH-IGX

E. Forest, "The Nonlinear Part of the Drift." May 21. 1941. I:S(; TECH NOTI:-16")

M. Furman. "Analytic Approximations for Synchronous

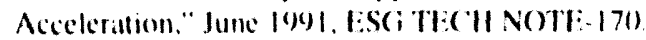

K.J. Kim, "Exploiting the Polarised Valture of Synchrotron Radiation." Workshop on Applicittion of Circularly Polarized Photons at Al.S with a Bend Magnet Surece June 11-11, 1991, LEST TECH NOTE-171

S. Krishnagupal, R. Sicmann (Sl.AC). "Ficld Calculation Algorithm for General Beam Dislrihutioms." July 29. I(5)I. FSC

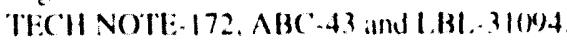

S. Lidiat, "Design and Performance lssues for the CDRL. Pree Electron Latser Resonator Cavity." August 22. 1991. FSC Tl:Cll NOTE-173.

J Bengtsson. "Trip Report PSI, Swit/erland." July I - 26. 1901. ESC; TECH NOTE-174

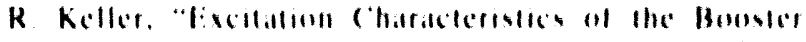

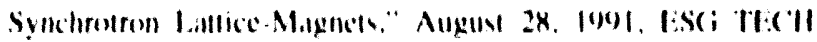
Nolli. 175

(i.A. Krafle. M Xice "Heam Breakup in a Superconducling

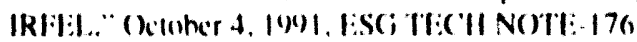

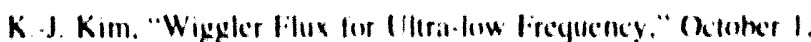

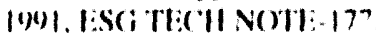

M. Furman, Y II (hin, J Eden, II.BI, W Karanech) I Tennyom, V. Ziemann (SLAC). "Clused Orini !)islention and the Beam-Beam Interaction," (C)on Rel ESS(i Nite-19)3.

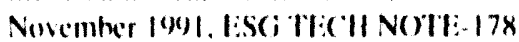

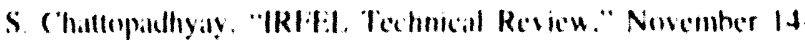
15. 1001 BSG II:C 11 NOTl:-170

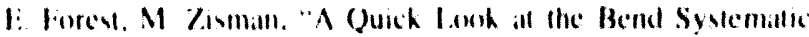

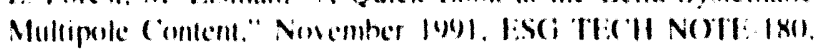
ABC .5 .4

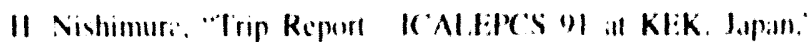
November 2-17. 1001. HSG TE( II NoTl:- $|8|$


Magnel." Jan 17, 1902. 1:SC THECII NOTE IX2

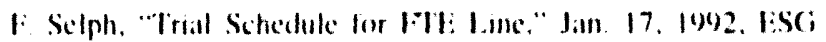
TECH NOTE-183

K.J Kim. "Oulline of the Talk "Peak Power Versus

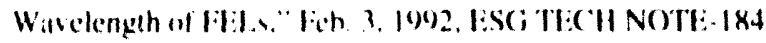

Y Funakoshi IKI:K) Y H ('hin. M Furman (l.BI.), W

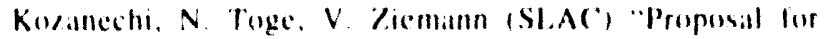
Experiments at Triblan on Beam-Beam lssues for fulure

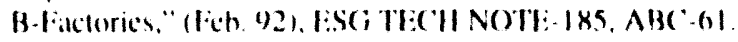

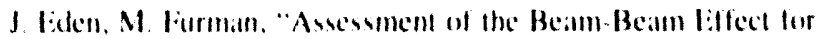

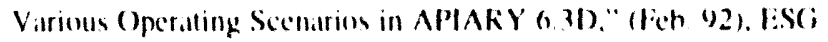
TECII NOTE- 186. ABC' 62

G. Lamberson, "The Beam limpedances Between Parallel

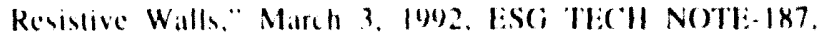
BLCON.102 
M. Meddahi, "Trip Report: loth International Conference on Computing Methods in Applied Sciences and Engineering, INRIA. Paris. France," February 6 - 17. 1992. ESG TECH NOTE-188.

W. Barletti. A.M. Sessler and L.-H. Lu, "Using the SL.AC twoMile Accelerator for Powering an FEL," March 15, 1992. ESG TECH NOTE-189.

A.M. Sessler, "Beam Conditioning for Free-Electron Lasers," March 10. 1992. ESG TECH NOTE-190.

ESG TECH NOTE-191, R. Donahue. Pending.

K.-J. Kim. "Trip Report: BESSY/Crossed Undulator Project \& EPAC 92. Berlin, Germany, March 21-29, 1992. ESG TECH NOTE-192.

R. Rimmer, "Trip Report: EPAC' 92, Berlin. Germany," March 21-29, 1992. ESG TECH NOTE-19.3

F. Voclker. J. Johnson. "Status of RF Feedback Kicker Design," April 27. 1992. 1ESG TECH NOTE-194 and ABC-69)

J. Byrd. "Resistive Wall Instahility at the ALS." May 1. 1992. ESG TECH NOTE-195 and L.SAP-122.

J. Bengesson. "Trip Report: ERCIM/Seminar on Parlial Differential fiquations and Group Thenry. Bonn, Germany," April 6-10, 1992. ESG TECH NOTE-196.

C. Kim. "Empirical Tune formulas for the ALS Booster Synchrotron," May 13. 1992. ES( T TECH NOTE-197 and I.SAP-124.

F. Sclph. "Timing Considerations in the Phase Jump Beam Compensation for the ALS Linac." March 10, 1988. ESG TECH NOTE-198.

W. Leemams, A. Sessler, and S. Chaltopadhyay, "Plasma Lens Experiments at the ALS Injector." April 8. 1992. ESG TECH NOTE- 19\%.

J. Corletl. "Higher Order Modes and Parasitic Lonses to the B. Factory RF (avities, May 1, 1992, ESG TIECH NOTL-20).

C. Lambertson, "Conductive Coating in Bump-Magned Tuhes." June 3, 1992, FSS F TECH NOTE-201 and I.SAP-126.

R. Alvis, R. Keller, "Storage Ring Sextupole Shunting," June 17. 1902. ESG THECHNOTL-2012.

S. Liciia, "Preliminary Design and Performance Evaluation for an XUV ALS Crossed-Undulator System," June 19, 1992, ESG TECH NOTE- 203

M. Furman. "Trip Report: Wilson Laboratory. Comell University, Ithaca, New York," June X-12, 1992, LSG TICH NOTE-2(14
G. Rangarajan, "Trip Report: 19th International Colloquium on Group Theoretical Methods in Physics, Salamanca, Spain," June 28 - July 4. 1992, ESG TECH NOTE-205.

A.M. Sessler, "Trip Report: Thomson Tubes, Paris, France; CERN, Geneva. Switzerland; HEACC'92, Hamburg, Germany \& LC"92, Garmisch-Partenkirchen, Germany," July 10 - August 1,1992 , ESG TECH NOTE-207.

E. Forest, "Trip Report: KEK. Tsukuba, Japan \& UVSOR, Okazaki, Japan," June 15 - July 20, 1992, ESG TECH NOTE206 .

C. Wang, "Envelope Equations for an Annular Beam," August 1992, ESG TECH NOTE-208.

M. Zisman, "Trip Report: HEACC'92. Hamburg, Germany \& CERN, Geneva, Switzerland," July 18 - 29, 1992, ESG TECH NOTE-20\%

J. Eden, M. Furman. "Further Assessments of the Beam-Beam Effect for PEP-11 Designs APIARY 6.3D and APIARY 7.5," September 1992, ESG TECH NOTE-210) and PEP-11/AP Note. $2-92$

S. Krishnagopal, "Trip Report: HEACC'92 \& DESY, Hamburg, Germany \& CERN, Geneva, Switzerland," July 17 - August 11, 1992. ESG TECH NOTE-211.

A.M. Sessler. "Trip Report: FEL'92, Kobe, Japan: KEK Tokyo, Japan; \& Workshop on Microwaves, Tokyo, Japan," August 23 - September 3, 1992. ESG TECH NOTE-212.

J.R. Eden, M.A. Furman, "Simulation of the Beam-Beam Interation with Tune Compensation," October 1992, ESG TECH NOTE-213.

J. Edighoffer, "Trip Report: FEL'92, Kohe, Japan \& ETL. KEK. JAERI, Japan," August 21 - September 2, 1992. ESG TECH NOTE-214

B.W. Littmann. "An Equivalent Circuil Approach to Determine the Wave Guide Loaded Cavity Q." Octoher 1992. ESG TECH NOTE-2.15

J. Eden, M. Furman, "Simulation of the heam-heam interaction for PEP-Il with unequal heam-heam parameters," October 1992. ESG TECH NOTE-216.

K.-J. Kim. "Trip Report to Kohe - Tsukuha - Takai-Mura, Aug 21 - Sept. 2," Octoher 5, 1992, ESG TECH NOTE-217.

B.W. Littmann, "MSC/EMAS" (A Handhook Summary) OCt. 7. 1992, LSG TECII NOTE-218.

M. Furman. "Scaling Rules for Uncqual Beam-Beam Parameters." Novemher 1992. ESG TECH NOTE-219. 
J. Byrd, J. Corlett, "Calculation of Multibunch Collective Effects in the ALS," October 20, 1992. ESG TECH NOTE-220, LSAP- 129.

W. Leemans, "50 MeV Beam Line Design: Progress Report," November 3, 1992, ESG TECH NOTE-221.

Y.H. Chin, "Trip Report: CERN, Geneva, Switzerland," August 13 - Octoher 19, 1992, ESG TECH NOTE-222.

M. Zisman, "Trip Report: CERN, Geneva, Switzerland \& Benalmadena. Spain," Octoher 25 - November 5. 1992, ESG TECH NOTE-223.

J. Byrd, "Simulation of the ALS Longitudinal FB System," ESG TECH NOTE-224.

J. Byrd, "Head-Tail Damping at the ALS," December 15, 1992, ESG TECH NOTE-225 and LSAP-1.30.

M. Zisman, "Trip Report: KEK. Tsukuha, Japan," Novemher 15-20, 1992. ESG TECH NOTE-226.

S. Chatlopadhyay, "Trip Report: HEACC"92, Hamburg. Germany \& LC'92, Garmisch-Partenkirchen. Germany," July 18 - August 3. 1992. ESG TECH NOTE-227.

M. Furman. "Trip Report: Benalmadena, Spain \& CERN, Geneva, Switzerland," Octoher 27 - November 7. 1992, ESG TECH NOTE-228.
S. Lidia, "SPEAR Beamline 5 Circularly Polarizing Undulator Magnetic Ficld. Trajectory, and Radiation Calculations: Ideal Behavior and Orf-Axis Effects," December 17, 1992, ESG TECH NOTE-229.

R. Rimmer, "Trip Report: KEK, Tsukuba \& Spring-8, Tokyo, Japan and POSTECH, Pohang. Korea," November 15-28, 1992. ESG TECH NOTE-230.

D. Rohin, "Trip Report: KEK. Tsukuba, Japan and UVSOR. Okazaki, Japan," Noveınber 15 - 25, 1993, ESG TECH NO'TE231 .

M. Furman, "Dynamical Beta Function and Tune Shift for PEPII." 1993. ESG TECH NOTE-232 and PEP-II/AP Note 4-93.

W. Leemans and J. Bengtsson, "Beam Test Facility Line Design: Specifications for Power Supplies and Magnetic Mcasurements." $1 / 12 / 93$, ESG TECH NOTE-233 and BTF Note-())2.

S. Chattopadhyay, "Experimental Program for the Beam Test Facility at LBL (FY'94-'95)," Jan. 13, 1993 (DRAFT), ESG TECH NO'TE-234.

R. Gough, "Beam Test Facility at LBL (Project Status Summary),"Jan. 13. 1993. ESG TECH NOTE-23.5. 
Please send ___ copy(ies) of the CEATER For $\boldsymbol{B}_{\text {EAM }} \boldsymbol{P}_{\text {IIYSICS }}$

Directory 1993 and future updates. My mailing lave is attached.

Please change my name/address. My mailing label is attached.

Name

Address

ATTACH MAILING LABEL

HERE

Please clip and mail this form to:

Centerkor B Bam Pursics

MS 71-259

Lawrence Berkeley Laboratory

I Cyclotron Road

Berkeley, CA 94720 


\section{Contents}



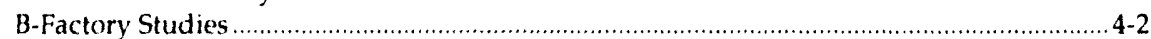

Conceptual Design Overview ................................................................................

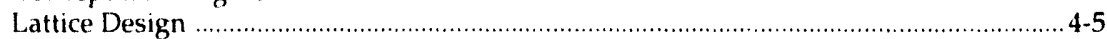

Beam-Beam Interaction Studies .......................... 4-5

Chemical Dynamics Research Laboratory ...............................................................4 4-7

CDRL: A Unique Combination of User Facilities ............................................ 4-8

IRFEL Design Progress ...........................................................................................4-8

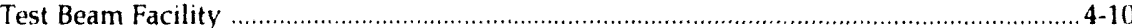

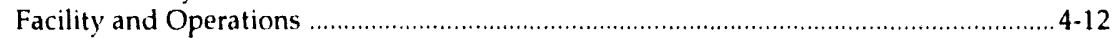

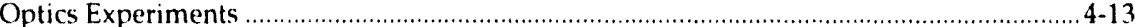

Bench Testing of HOLD $\quad 4-13$

Advanced Optical Diagnostics for FELs ............................................................... 4-14

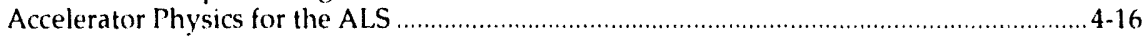

RF Measurements and Feedback Systems ................................................................... 4 -16

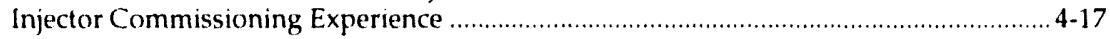

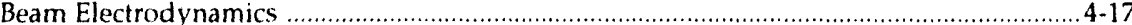

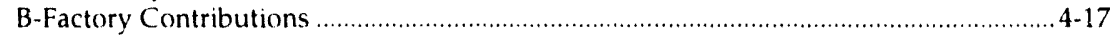

Fermilab Antiproton Cooling System ................................................................. 4-19

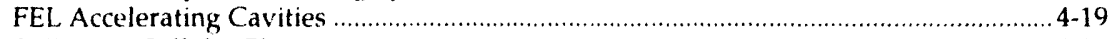

High-Energy Collider Physics ................................................................................ 4-19

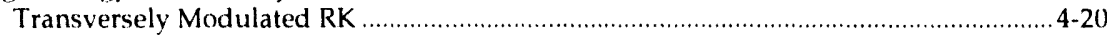

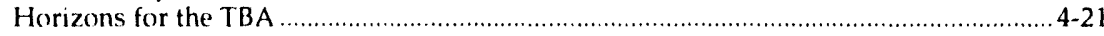

Beam Conditioning ..................................................................................... 4-22

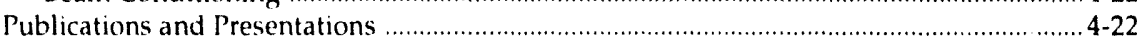

(Reprinted from Accelerator and Fusion Research Division: 1992 Summary of Activities, LBL-33377 (December 1992), Chapter 4) 


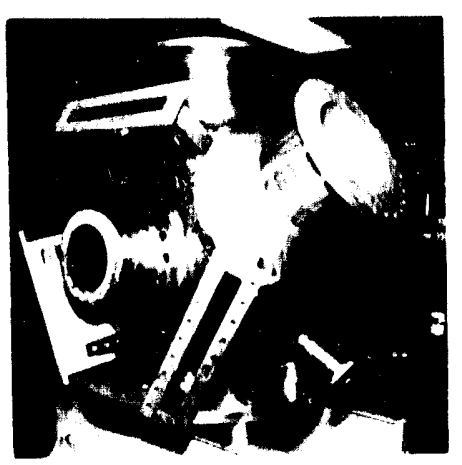

4.

\section{CENTER FOR BEAM PHYSICS}

$\mathbf{T}$

WO MAJOR ACCELERATOR-BASED INITIATIVES are being assisted by the Center for Beam Physics," a divisional center that performs multifaceted exploratory studies of the physics of accelerators and beams. PEP-II, a proposed B-meson "factory" based on the Positron-Electron Project ring at the Stanford Linear Accelerator Center, has been the subject of ongoing research. Meanwhile, a long-standing interest in free-electron lasers and highbrightness electron and photon sources has led to the detailed design of an infrared free-electron laser (IRFEL), which is proposed as part of the Chemical Dynamics Research Laboratory. The IRFEL investigations have led to productive collaborations with Stanford University, Brookhaven National Laboratory, the Continuous Electron Beam Accelerator Facility, and TRW, Inc.

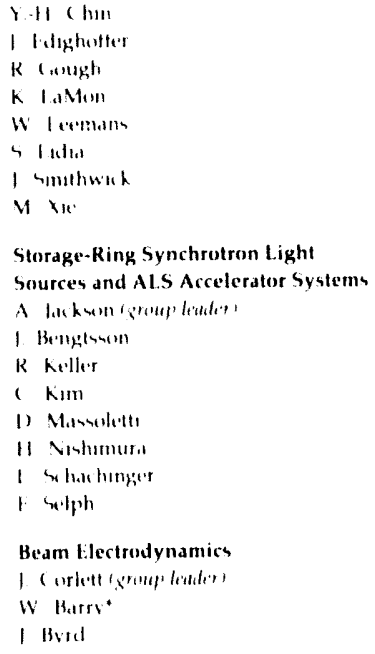

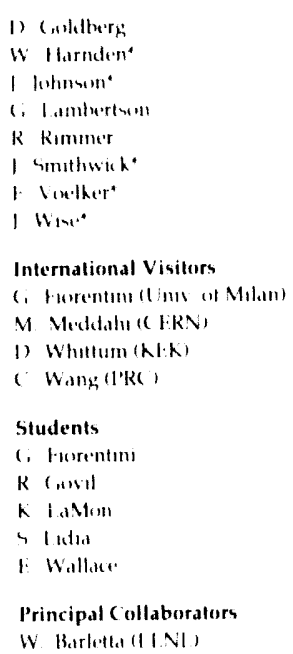

W. Barloth (l I NI)

\author{
Ben $/ \mathrm{N}(\mathrm{BN})$ \\ I burtan (4) $A$ ) \\ 1. Whlla IC I. BAI \\ C HormatikW \\ 1) Hitlon ic allew

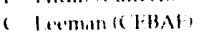

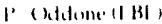 \\ A cxhwettman frtantond \\ (c) Ghand 11 BI 1 \\ (. Wentesiskion $111 \mathrm{VI}$ \\ Technical Support \\ 1) Massolett \\ 1 smothwik \\ 1 Win. \\ Administrative Support

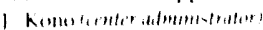 \\ 1. Alkumblowe \\ $M$ combon \\ M Mara Am \\ 1) Mirett

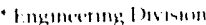

\footnotetext{
- The Center was formerly known as the lixploratory Studies Ciroup.
} 


\section{CENTER FOR BFAM PIIYSICS}

To meet the technical challenges of these initiatives and to generally enhance LBLis capabilities in particle- and photon-beam research, the (enter made several additions to its experimental capability in 1992 and early 1993. In addition to upgrading the beam electrodynamics laboratory and setting up an optics lab. the detailed design of the Advanced Test Beam Facility was finished and construction was started. This facility will get "double the money's worth" out of the 50-MeV ALS linac by using it for beam physies experiments during the considerable spans of time between the Als injection cycles. Many experiments are possible; immediate plans include generation and detection of $x$-ray pulses as short as tens of femtoseconds a long-standing interest ) and focusing of a beam by using plasma lensess.

Members of the Center have been involved in the Als project from the outset and make up its technical core, the Acceleratur Svstems (iroup. With the design of the accelerators complete, they have continued to play major roles in guiding construction, programming the control svstem, and commissioning.

Research continues in accelerater theory, nonlinear dymamics, and fundamental FFL physics. The lligh-Energy (ollider l'hysics group continued its long-range Twe-Beam Accelerater research. The Beam Flectrodynamics group contributed to and supervised the PleP-1l rf and feedhack design offorts. It also contributed signiticantly to rf, impedance, and feedbach work at the Als, and worked on beam-cooling improvements for the Tevatron's antiproton source.

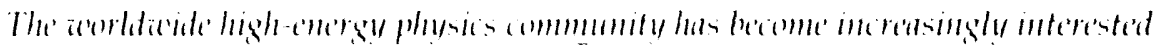



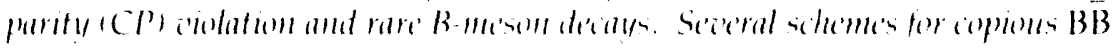

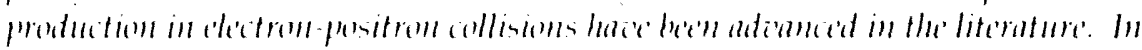

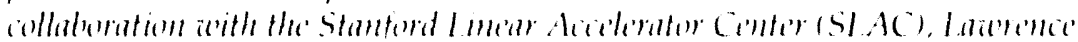

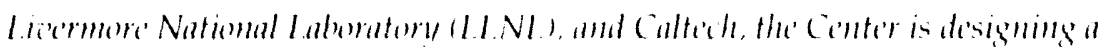

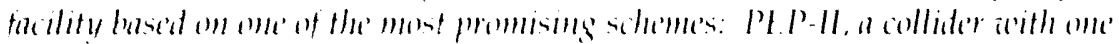

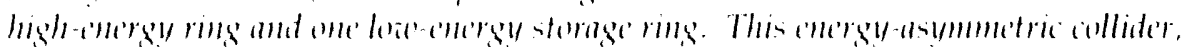

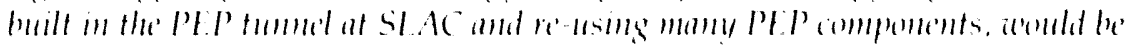
scientifically and eronomically attratiote.

During 1942, the PEE'-II collaboration continued refining the design of a $B$ factory in which a 9 -ciel' electron beam in PlEl' collides with a 3.1-Ciel positron beam circulating in a new storage ring. The new low-energy ring will be of the same circumference an PEP and will be mounted above it in the existing tunnel, as shown in figure $4-1$. The chosen energy combination reaches the $Y(4 S)$ resonane, at which $B B$ pairs are produced in the abundance reguired for the study of ('Prolation (sidelme. The challenge in the design of a B factory is 10 reach an initial luminosity of $3 \times 10^{3} \mathrm{~cm}^{2}-5$, which is more than an order of magnitude bevond the luminosities achieved to date in electron-positron colliders.

\section{Conceptual Design \\ Overview}

\section{B-Factory Studies}


Higure 4-1. The proposed asymmetric B factory, PLP-11, would be built in the Positron-Electron Project tunnel and would use a substantial amount of the existing hardware for the P'El' collider. Recent I.BL. work in the multi-institutional P'EP-II collaboration has emphasized refinement of the magnetic-lattice designs of the two rings and extensive studies of beam-beam interaction. IArtist's impression courtesy SL.AC)

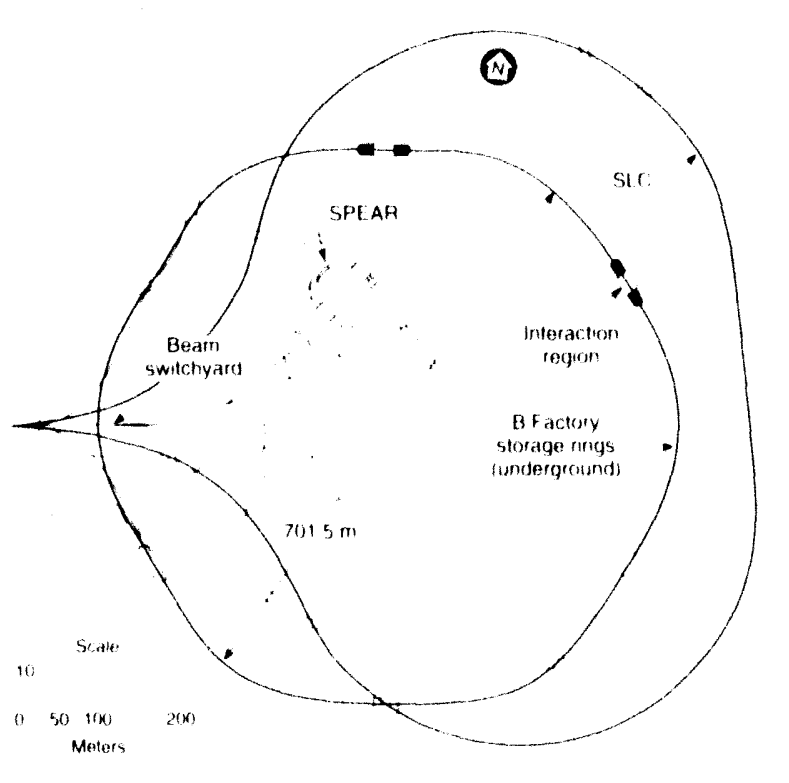

$\times B 1902.5762$

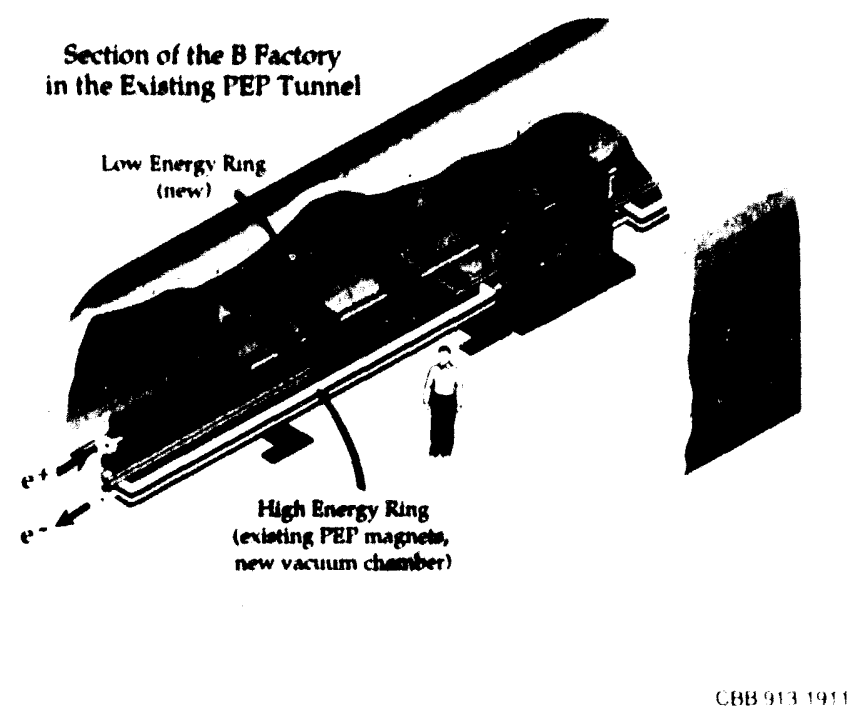

In principle, all the relevant parameters the ratio of the cross sections of the two beams, the beam currents, the beam-beam tune shifts, the beam energies, and the vertical beta functions at the interation point-are adjustable. In practice, however, the beambeam tume shift cammot be increased bevond a certain value, which has been determined experimentally in many colliders to lie in the range of 0.02 to 10.06 . Similarly, a collision energy at the $r(4 S)$ resonance implies that the product of the beam energies must be 28 ciev. Thus, only three parameters the beam-size ratio, the beam current, and the vertical beta function at the interaction point-are fully at the discretion of the acceleratur designer

The chosen luminosity, $3 \times 11^{1:} \mathrm{cm}:{ }^{1}$, has been shown to be adepuate for the study of the key physics issue, ( I' riolation. Given the limitations cauned hy the beam-beam interaction which we take for our design to correspond to a maximum tume shift of 19.113 .... a substantial increase in luminosity implies that the high-current beam must be divided inte a large

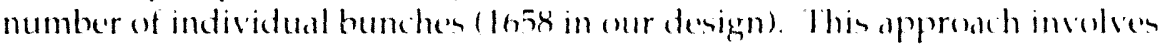
a design in which the simgle bumeh parameters lemittames, bumch length, current, tume shift, ets.) are well within present proctice for colliders. ()ur chose of 1658 humches lies in a ste midille ground between the extremes it does not exacerbate coupled-bunch instabilitien, nor does it have problems with single-bunch offects. 


\section{CINTHR POR BEAM PIMSICS}

For l'El' to serve as the high-energe ring, several of its systems must be significantly upgraded to deal with these isstees. Foremost among these are the rf and racuum systems. The high luminosity leads to extreme heat loads caused by synchrotron radiationi, a great deal of engineering work has been performed by our collaborators to solve the resultant cooling and vacuum problems. There are also implications for the rf system of the high-energy ring. It will operate at a frequency of $476 \mathrm{MHz}$ to phase-lock the stornge ring $\mathrm{rf}$ system to that of the 2856-MHz injector (the "two-mile linac" that is also used to inject the Stanford Linear (ollider). This choice of frequency minimizes injection phase errors, which contribute significantly to the power demands of the multibunch feedback system. The rf system will consist of 21 single-cell cavities; the cavity design itself is aimed at minimizing the higherorder-mode impedance contribution of the rf system.

The interaction region is designed in such a way that the beams collide head on. During 1992, a great deal of effort has gone inter studying the beam-berm interaction and into refining the lattice designs of the two rings and the interaction region. These three subjects are closely related.

\section{B Decays and CPT Symmetry}

Judging particle interactions by the standards of the familiar, macroscopic world, one would think that if a process and the participating objects were replaced either by their antimatter equivalents or by versions of themselves as seen in a mirror, the rate of the process would remain the same. It seems equally intuitive that reversing a process would yield the original participants, much as though one were running a movie in reverse and watching the actors run backward in their own footprints.

But on the scale of subatomic particles and the quarks that make them up-a scale where the "weak interaction" becomes the strongest of forces-the first two rules, called "conservation of parity" and "charge conjugation," are not necessirily obeyed. Not even Cl' symmetry, which combines both rules, necessarily holds true. The remaining variable is time; we are left with CPT symmetry-a scheme in which $\mathrm{C}, \mathrm{P}$, and $\mathrm{CP}$ symmetry violations can occur, but only if the arrow of time is allowed to take a different course when reversed, going back $t 0$ a different beginning.

Thus far, $\mathrm{CP}$ violation has been observed through asymmetries in the decay modes of the neutral $K$ meson and its antiparticle. The $K^{0}$ and $K^{0}$ contain an unusual quark, the "strange" quark, which is not found in the group of quarks that make up ordinary matter. The $K$ decays in a wide variety of fashions (it is axiomatic that every decay mode that is not explicitly forbidden must (xcur eventually). In a few of these modes, the $K^{\prime \prime}$ decays a few tenths of a percent differently than the $K^{\prime \prime}$, a sign of $\mathrm{CP}^{\text {P }}$ "olation. But studies of the $K$ system have left many questions unanswered about the mechanisms and magnitude of $\mathrm{CP}$ ' violation.

The B meson, which contains a different unusual quark ("bottom" as opposed to "strange"), is predicted by the Standard Model of Particles and Interactions to have asymmetries as great as $30 \%$ in some rare decay modes. This makes it a very promising candidate for $\mathrm{Cl}$-violation studies. However, the branching fraction-the proportion of pairs that will not only decay through the unusual modes but also violate $\mathrm{CP}^{\text {? }}$ symmetry in doing so-is only about $10^{-4}$ to $10^{-5}$. Therefore, about $10^{7}$ to $10^{4} \mathrm{BB}$ pairs will have to be produced to get good $\mathrm{CP}$-violation statistics. This requirement, implying the need for many $\mathrm{e}^{+} \mathrm{e}$ collisions, brings us to the luminosity frontier of accelerator physics, whose technical challenges are described elsewhere in this chapter.

The ultimate goal of this research is to enhance the Standard Model-today's partial theory of the building blocks of nature and how they interact-or replace it with a new, more-satisfactory theory. In either case, CP violation will have to be better quantified, and its origins will have to be explained. The present Standard Model does not disallow $\mathrm{CP}$ violation but does not explain it either. These studies also have ramifications beyond particle physics.

In 1967, not long after the discovery of $\mathrm{CP}$ violation, Andrei Sakharov pointed out that it might explain one of the long-standing riddles of cosmology: why the universe was not born with equal, evenly distributed quantities of matter and antimatter that would annihilate each other whenever they interacted. For some reason, the laws of nature appear to prefer matter over antimatter-a phenomenon that makes possible the physical universe we see every day. Such will be the implications of the research at PEP-II. 
Lattice Design

\section{Beam-Beam Interaction Studies}

The basic littice desighs on both rings were completed in earlier years; the primary effort during $f(x) 2$ was, and remains, optimigation of the lattices (particularly that of the low-energy ring). Using simulation fouls that we had

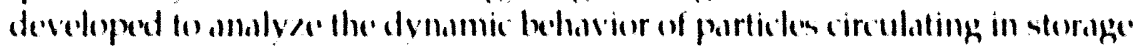
rings, we found that the lowe energy ring was not as dynomically stable as the high-energy ring. Thene took are bolsed an single-particle tracking, and fully

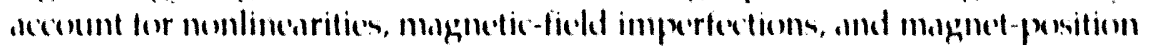

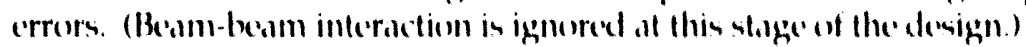

Significant strides have been made toward understanding the problem Gient dynamic behavior reguires a stable aperture al least lo limes the natural beam sies. I fowever, ane commot make a penitron beam that is af the same yuality as the chectrom beam; its natural emittance and energy spread are each roughly 1.5 times larger. Therefure, all else being evenl, ane must acheve a larger aperture in the lewe-energe ring than in the high-encegy ring. In addition, the stromger locusing of the low-energy ring reguires stronger sextupole magnels in order to correct the linear chromateity. As o result of this correction, the low-energy ring has more monlinear chromaticity, as well as a greater tune shift under amplitude variations, than the high-energy ring All this reduces the avilable dyommio apertare.

()ur prenent resulte show that, tecontrol thene effects, it is mecossary to have a lacil chromaticity-compensattent seheme in hoth planes mear the

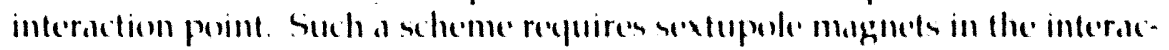
tion-region straight section. They must be placed in sted a wily as te correst the chromaticity arising from the interaction-regions guadrupole magnets withent generating higher-order tume shitts. ()ur simulations have shown this scheme to be the best methed of improving the dynamic aperture. As we approsth a final design for the interaction region, the challenge we face is to implement this scheme while respecting the geometrical constraints imposed by the existing tomnel and by the relative pessition of the twe rings.

While working on the hattice design, we also intensified our studie's of bermbeam edfects. We have made a great deal of progress on a simulation approach that complements the lattice studien by providing detailed simulation of beambeam dynamice but negheting magnet nomlinearities. These beam-heam stadies are based on multiparticle tracking simulations, so they repuire much more computation than the single-particle dynamic-aperture studies. Ideally we would like to combine the two approsiches into one comsistent whole; we infend tedevelop the necensary fords for this anitication during 1643.

Most of the'se studie's were based an multiparticle simulations in the linear-lattice approximation. In the angoing detailed-design effort, a gored deal af actelitional proggress has been made an various isstes, including the exintence of an adeywate "working perint" in the multivariate parameter space, adeguacy of the beam separation at the parasitic collision points, beam-beam interaction during injection, departures from full equality of the four beam-beam parameters, and an experimental proposal at TRISIAN" W test the effects of the beam-beam foree on the closed orbit.

In search of a working point, we performed tume scans onver a wider regien of the teme plane than we had in the initial effert that produced the

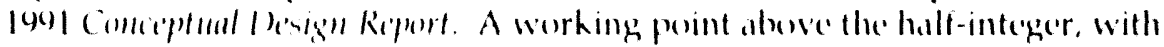




\section{( INIIR IOK BIIAMIIISGLS}

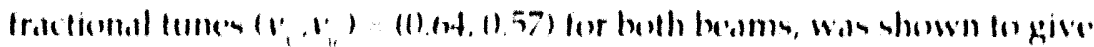

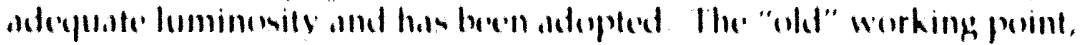

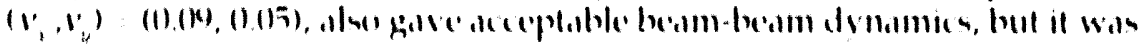

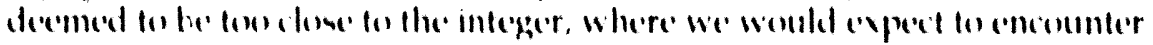

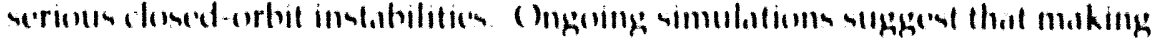
the working perinte slightle diflerent fer the two besme will give even better perturmance.

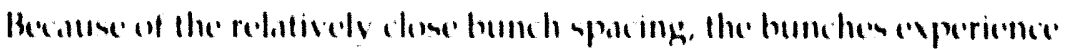

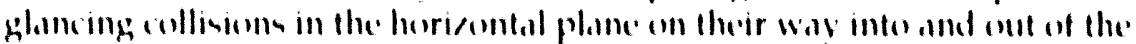

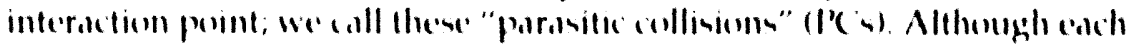

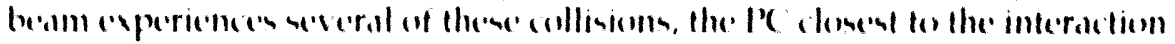



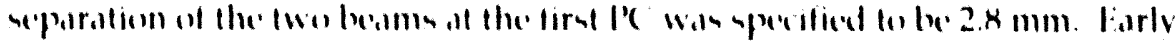

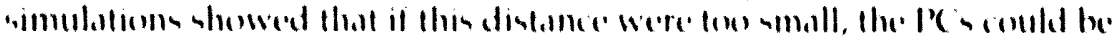

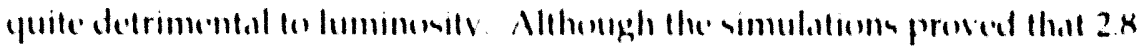

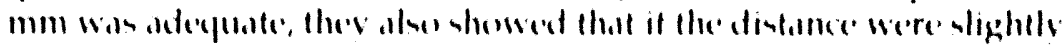

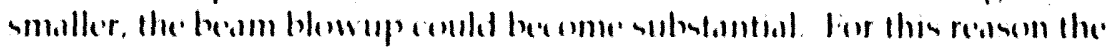
design of the interaction regien has been upgraded te proside a much more comforlable 3. 5-mm separation.

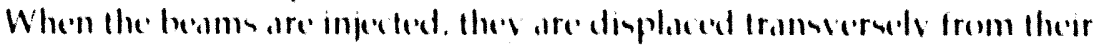

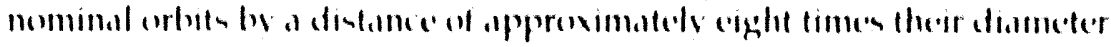

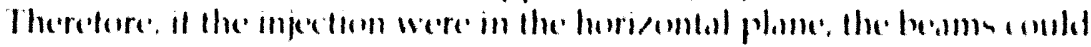

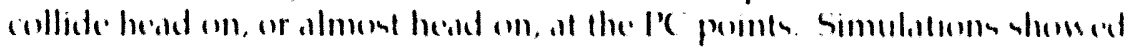

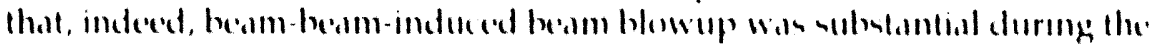

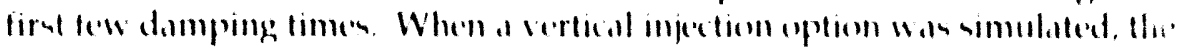

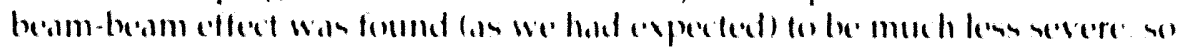

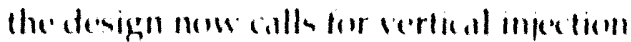

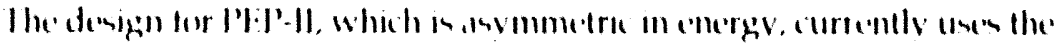

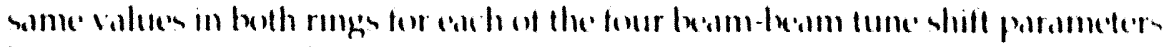

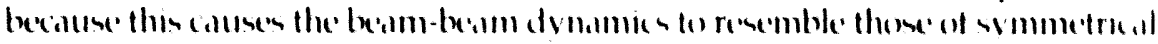

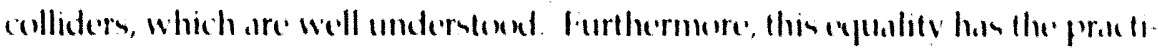
cal advantage of comstraining many other parameters. Recent simulatums

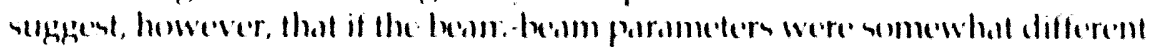

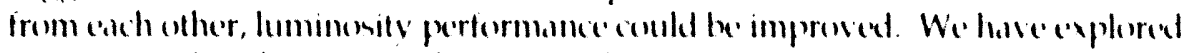

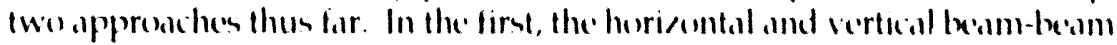

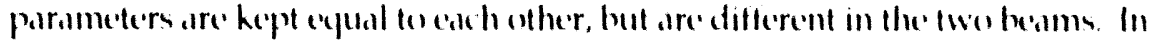
the second, the horizontal and vertical heam beam parametere are different trom each other, but are kept expal for the two heams

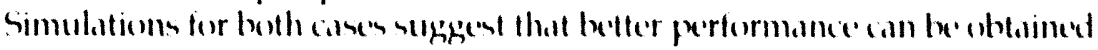
in the first come by uning a slightly smaller beam-beam parometer for the pesitron beam and in the serond cane by using a slightly umaller vertical beam-

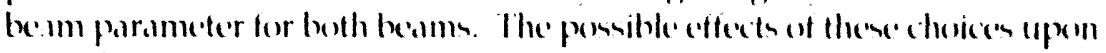

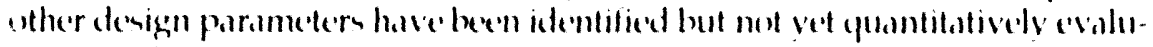
ated. Undoubtedly ame combination on the two approsches, or a third approsch in which all four beam beam parametern are ditferent, will prove to be

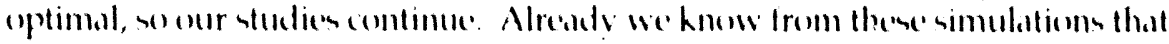
the besm-besm dynamion remain well behased when the tene parameters are not cyual, indicating that, at least, our initial reguirement fere e'puality in the'se'

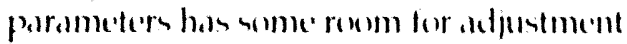

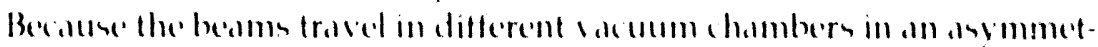
ric collider, the besm orbitn mear the interad tion point need to be controlled to

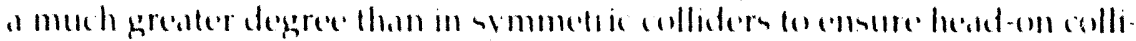




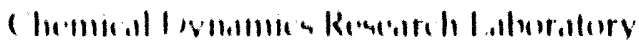

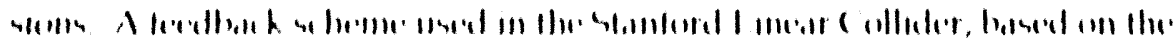

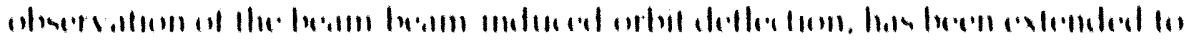

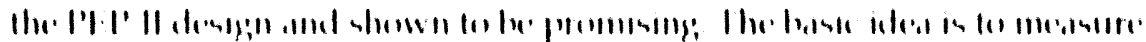

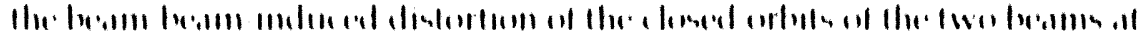

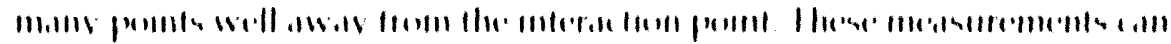

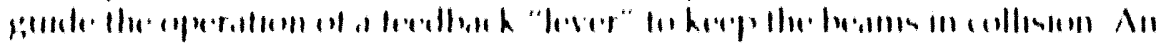

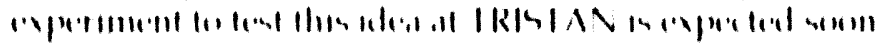

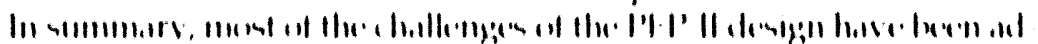

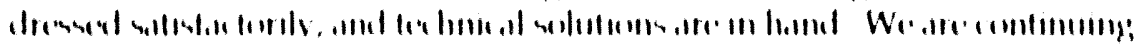

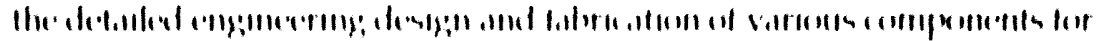

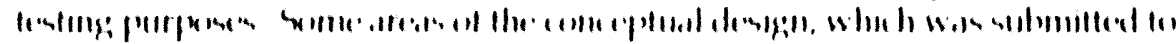



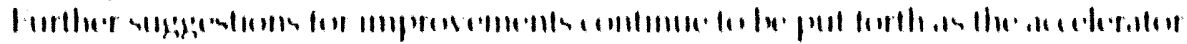

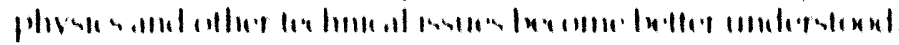

\section{Chemical
Dynamics Research \\ Chemical
Dynamics Research Laboratory}

\section{Rescorch lrospiects}

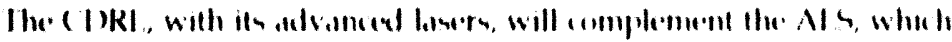
"1p

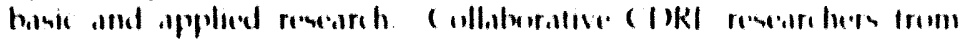

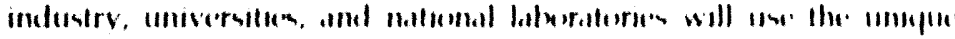

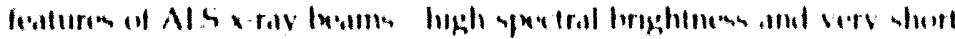

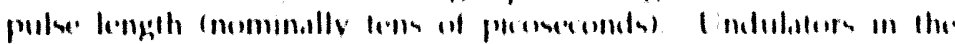

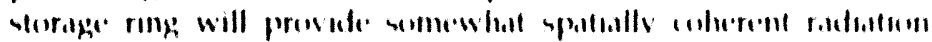

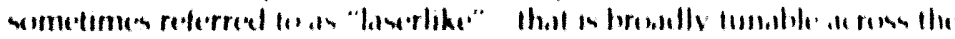

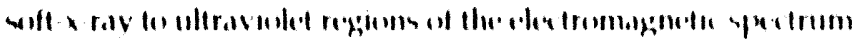

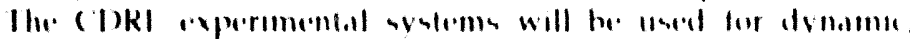

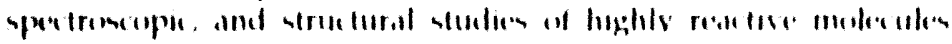

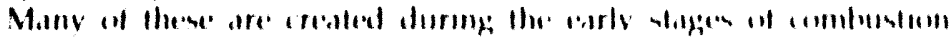

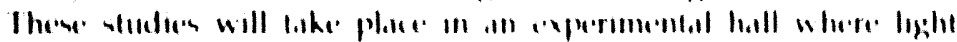

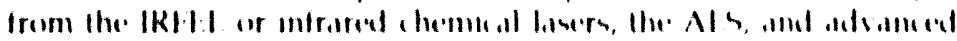

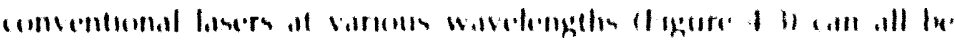

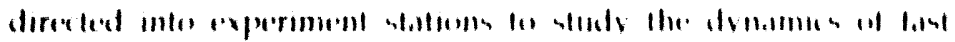

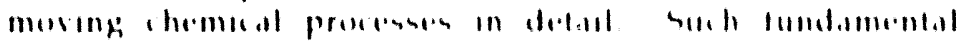

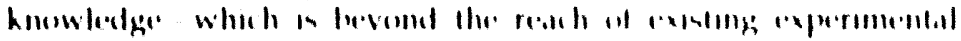

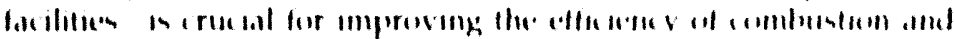

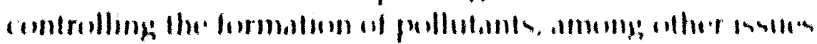

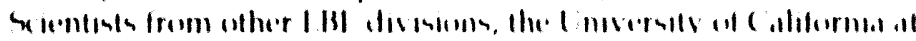

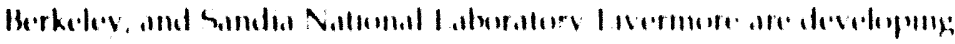

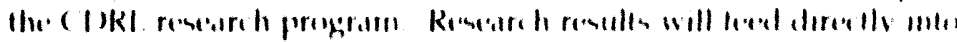

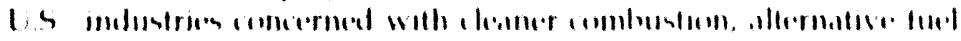

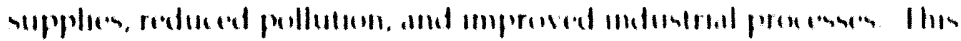

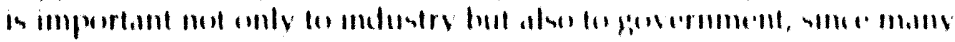

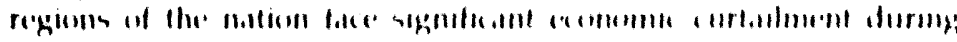

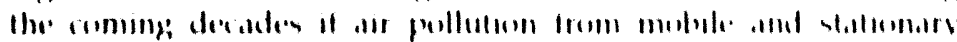

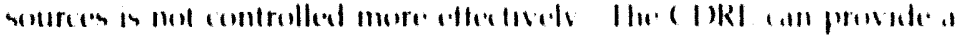

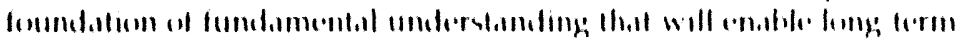

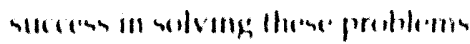

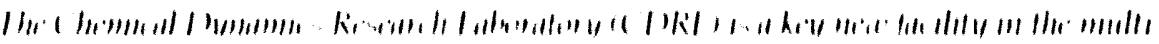

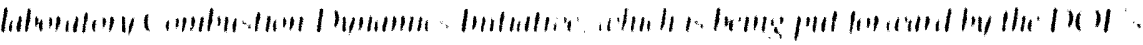

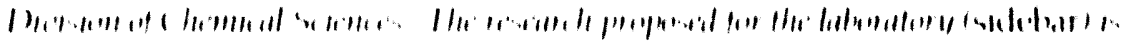

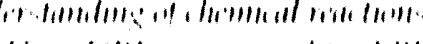

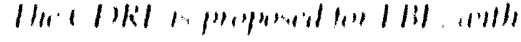

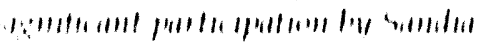

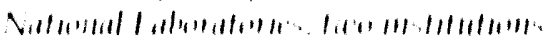

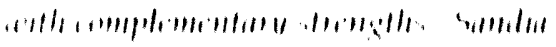

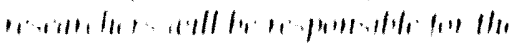

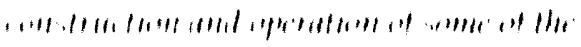

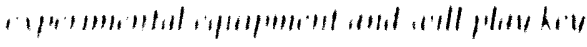

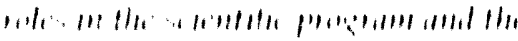

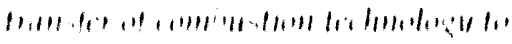

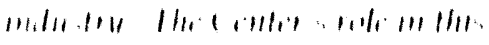

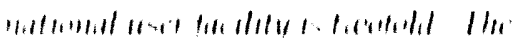

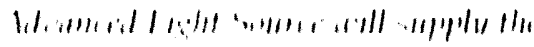



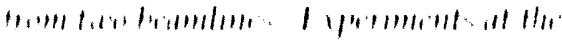

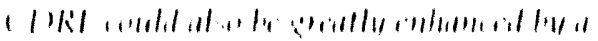

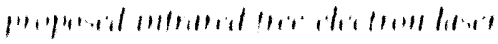

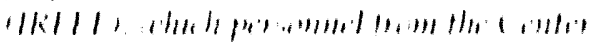

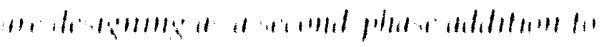

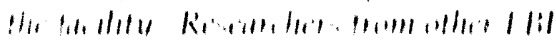

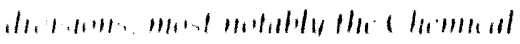



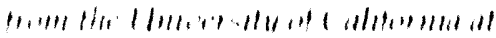

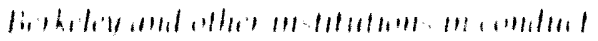

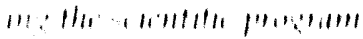




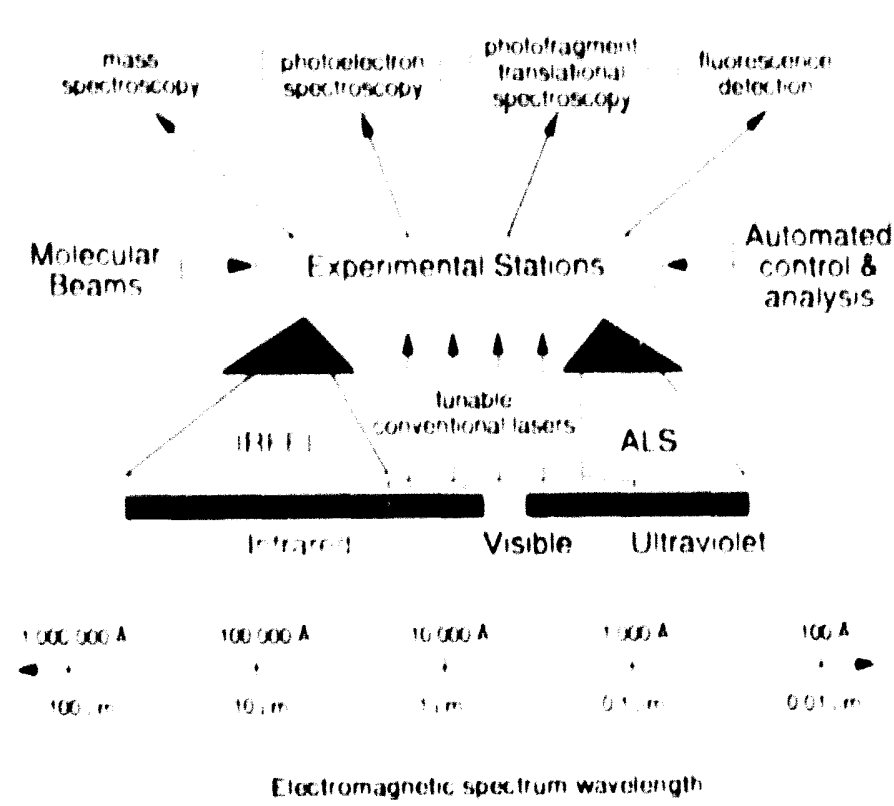

r. BB? $(x), 6,)^{4}$
Iigure 4.3. A key Io the scientific potenlial of the CDRI. iv the unprecedented integralion of several technologies over a wide spectral range. Tunability. synchronication capabililies, and time resolution on the order of plecosecond are among the other importanl features of the proposed facilities. The lowerenergy region of the infrared idifferent portions of which are of intereal for different kinds if researchl could be covered with chemical lavers; the proposed IRHI.L. would offer broad lunability over a wide range, in addition lo high power.

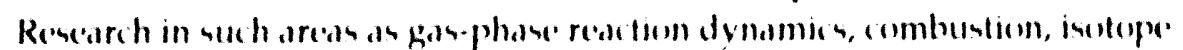

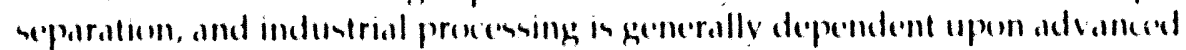

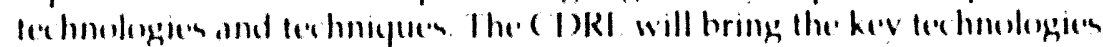

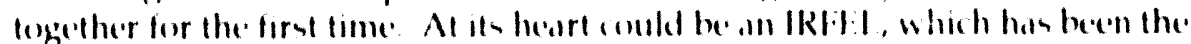

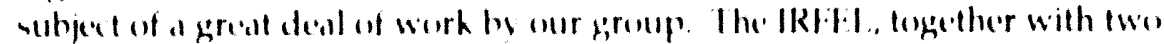

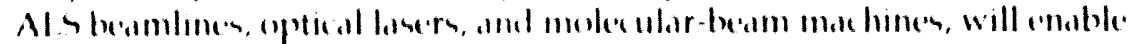

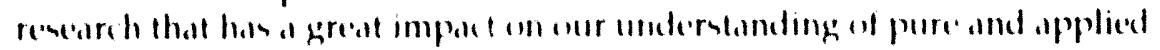
ihemical denamics

The ( I)RI will ocoupl a mew building adjocent to the Al S so that

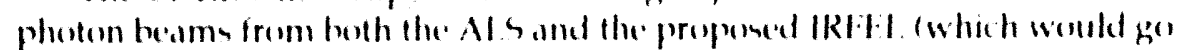
in a shelding vallt in the basement of the mew building san he delivered to the experimental stations. Ihe Als heamlimen have been another area of stude and develupment by our group. in collahoration with the Als stafl

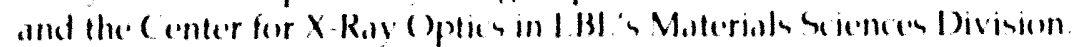

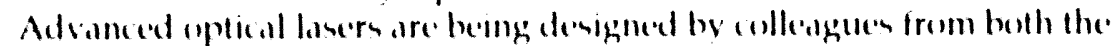
Lniversity of ( alifornia at Berkeley ( hemintry Department and Sandia

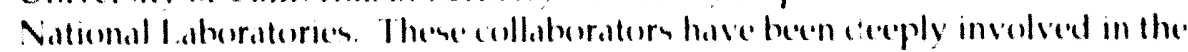
design ent experimental facilitien and in the develepment of the resedreh program

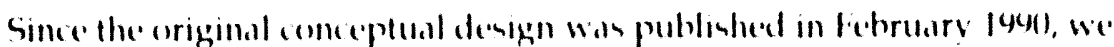

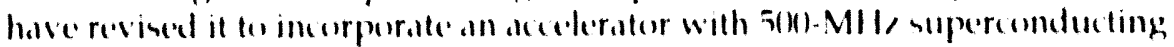
rf cavitien (lighure +2). The primery reasen for the mowe from the lessexpensive rowen-femperallure lechnolege was the user reyuirement for photen-beam wavelength stobility. Their reguirement for stobility fo within



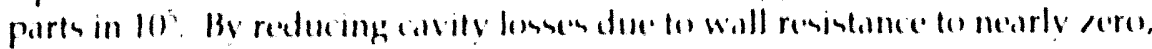

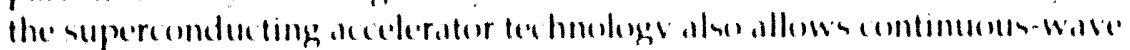

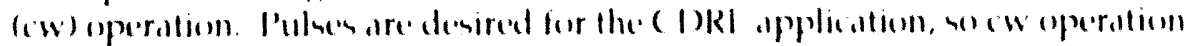

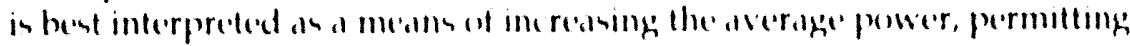
moredata to be whtanted in a given time

\section{(I)RL: A Unique Combination of User Facilitics}

IRIFI. Design I'rogress 
Figure 4-2. Recent thinking about the CDRL. IRlLL points toward a new design hased on 500)-MIte nioblum. litanium auperconducting linac structures. The superconducting technology allows continuous operalion (wilh various pulsed modes and the ability to serve multiple experiments) and lends ilself to a recirculalion loop. Downatream of the acreleralor apparalus shown here, coherent infrared radiation is produced with a varlable-gap undulator and an optical cavily wilh a broadly tunable hole-coupled outcoupling scheme. Also shown here is an indicalion of how the new design might fil in the basement shielding vault of the CDRI building proposed carlier; the beam dumps are shown but the cryogenic systems, which would be outside the vaull, are not.

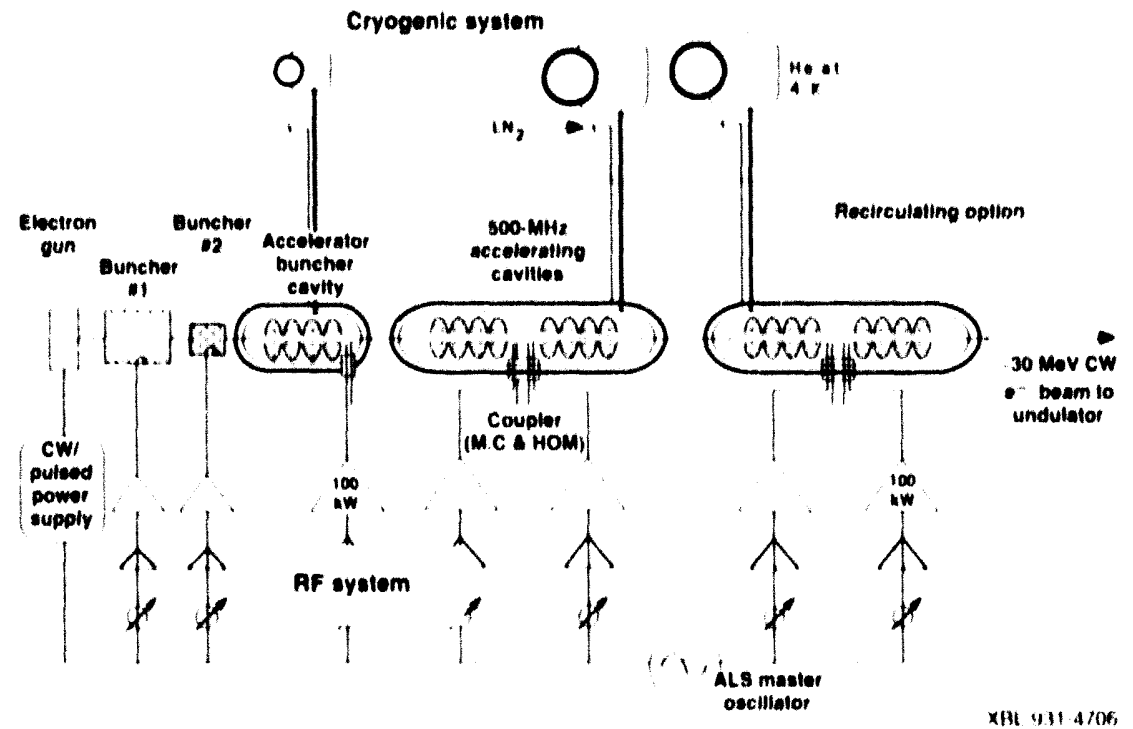

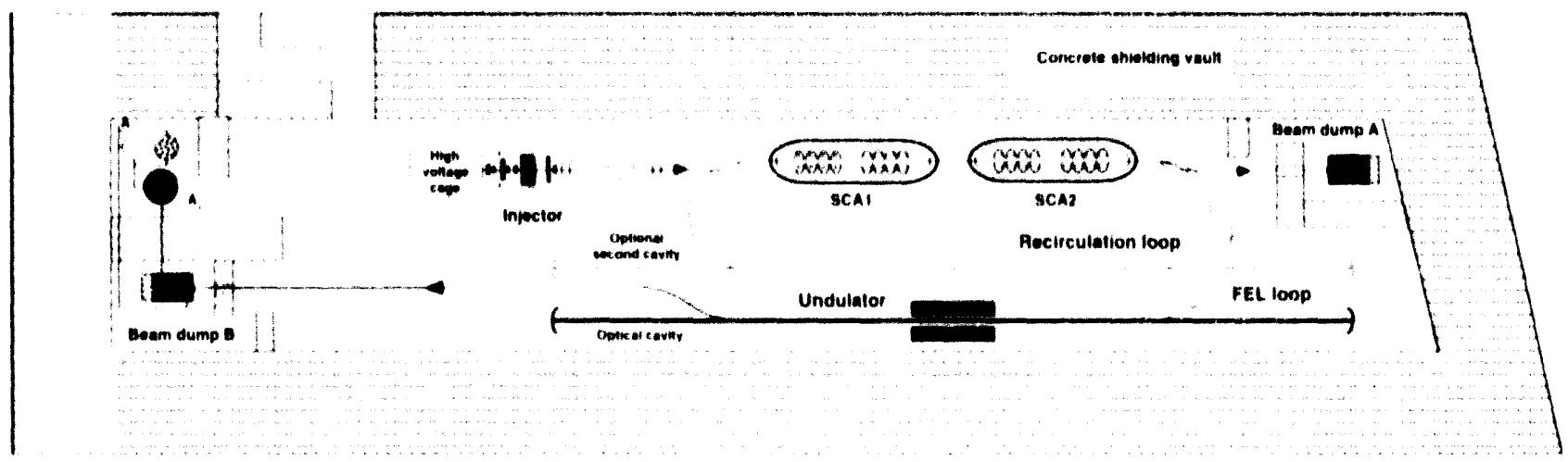

x(31 931.470

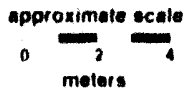

The pulse train can be tailured to meet the experimenter's needs in a variely of ways, indeding synchronization with the Als pulses. This flexibility will allow simultaneous service to multiple users. The IRIILI's micropulse duration is 33 pes, with a repetition rate of o.6 Mlle. Infrared radiation is produced with a variable-gapp undulator alld a $24.6-\mathrm{m}-\mathrm{l}$ optical cavity with a broadly tunable outcoupling scheme. Table 4 -1 summarizes the parameders of the new IRII:1 design.

P'articular attention was poid to stability and lumability, which are cructal for the users' needs. A detailed andysis of the effect of the electron beam fluctuation upen the optical performane was corried out through anolytic calculation and numerical simulation. Also stludied were various sources of fluctuations in the gun, bunchers, and accelerating sections, as well as feedhack and feedforward sehemes to reduce these fluctuations. The superconducting dengen proved superior 10 the 109() room-temperature design, which had an clectron-beam energy stability of I port in 10'? Thus the 5010 Mlle superconducting design appears to be the best chosce for our purposes. 


\section{CENTER FOR BEAM IPHYSICS}

Table 4-1. Some Characteriatics of the Proposed CDRL IRIEL

\begin{tabular}{|c|c|}
\hline \multicolumn{2}{|l|}{ Accelerator } \\
\hline RF fresfuency (MII) & 510 \\
\hline Maximum energy $(M(V))$ & -55 \\
\hline \multicolumn{2}{|l|}{ Micropulse } \\
\hline liwlIM energy spread & $0.35 \%$ af $55 \mathrm{MeV}$ \\
\hline FWIIM duration $(p)$, & .37 \\
\hline Reputition rate (Mils) & 6.6 \\
\hline \multicolumn{2}{|l|}{ Output } \\
\hline Wavilenglh $\lambda(\mu \mathrm{m})$ & 350 \\
\hline linewidth & |ranstorm-limitert \\
\hline Bandwidth stability $\delta \lambda / \lambda$ & $10+4$ \\
\hline Intensity stobility 8 l/ & 0.1 \\
\hline Average power (W) & $a(n)$ \\
\hline
\end{tabular}

The FEL design must provide wide wavelength covelage while minimizing operational interruptions. At a fixed electron energy, the wavelength can be funed by about a factor of two by varying the magnet gap of the undulator. For rapid fine tuning, we can change the electron beam energy by $+1 \%$, varying the photon wavelength by $+2 \%$. By operating the accelerator at any of four different energies $(55.3,39.1,27.7$, or $19.6 \mathrm{Mc} \cdot \mathrm{V})$ and using the'se tuning techniques, we can cover a wavelength range of $360.50 \mu \mathrm{m}$.

The beam reaches about $30 \mathrm{MeV}$ in its first pass through the pair of superconducting $r f$ cavities. With an extra, in-phase recirculation pass, it reache's about $55 \mathrm{MeV}$, greatly extending the short-wavelength capability of the IRIELL. In another operationsl mode, the recirculated beam could instead be introduced into the cavities 180 out of phase with the rf. This would decelerate the beam, putting its power back into the rf cavities in a sort of flywhed effect for use on the next pulse. The electron beam, and hence tie optical bean, would become quite powerful.

Our work on the recirculation scheme is beginning to address such important issues as isochronous beam transport and safe dumping of energetic, intense beams. We and our potential users are also studying the scientific implications of operation at this high power. The design effort has spawned several experimental programs, including an LBL-Stanford collaboration on development of novel diagnostics for FEL optical pulses, a Stanford-LBL-TRW-BNL collaboration on optimization of superconducting cavities for FEL, an L.BL. experimental study of hole-coupling and resonator modes, and a joint L.BL-CEBAF test bed for demonstrating superconducting IRFEL technologies.

The Al.S injection complex includes a trateling-wane lima that produces a 50 - MeV Test Beam Facility

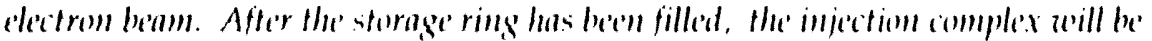
idle for the useful lifetime of the stored beam, which is axpested to be seteral hours. A variety of interesting axpriments conld be conducted with that beam, including plasma focusing, tests of accelerator structure's, and ge'meration of "chirped" photen

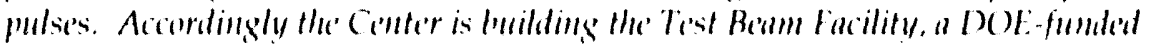

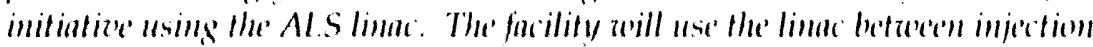

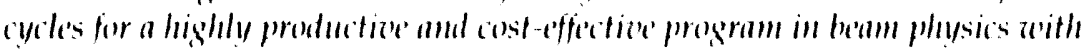
iminimal disruption to Al.s operations. 
Research Program
Many interesting experiments could be performed with this conveniently avalable, short-pulsed, low-emittance clectron beam. These two investigations are planned for the initial research program: a plasma focus and the generation of short $x$-ray pulses through Compton scattering.

When a relativistic electron beam passes through a plasma, electromagnetic interactions focus the beam. To date, most work with the plasma-focus concept has involved thin "lenses." Continuous plasma focusing with thick lenses holds the promise of overcoming the so-called Oide limit-a fundamental limit of focusability arising from statistical emission of high-energy photons in a sharp focusing bend. (Our plans include a proof-of-principle test and systematic exploration of plasma-focus ideas generated at our Center. (One of the ideas is a long, continuous plasma focus in which diaphragms and differential pumping combine to taper the plasma density. The density will be tapered from about $1 \times 101^{11}$ to $5 \times 10^{12} \mathrm{~cm}^{3}$ over a length of $0.5 \mathrm{~m}$. We hypoithesize that, at $50 \mathrm{MeV}$, such a device could focus a beam with a 3-mm cross section into a 400 - $-\mu \mathrm{m}$ spot. Our scaled proof-of-principle work will involve plasma lengths ranging from $10 \mathrm{to} 5() \mathrm{cm}$, with density tapering from about $1 \times 10)^{11}$ to $5 \times 10^{13} \mathrm{~cm}^{3}$ over that distance.

Two requirements must be satisfied for an effective plasma focus: the plasma response time must be short empared to the pulse length, and the plasma return currents within the beam must be small. We have calculated parameters for a number of experiments that can be performed using the 5()MeV inje. .r; they will allow corcful study of these requirements in both underdense and overdense plasmas. Furthermore', a study of the effect of plasma return currents on the effectiveness of the focusing can provide insight inte the usefulness of plasmas in reducing beam-beam interaction.

The design of the plasma source and diagnostics is currently under way. Two candidate sources are an rl discharge source and a photoionization source driven by an excimer laser. The plasma properties will be medsured using Langmuir probes and a 65-(i) Michelson interferometer.

Another experiment at the facility will use the AI S linac's beam for an ALS-like purpose: the generation of x-rays. Today, the shortness of photon pulses that are produced by either interaction with a magnetic field (synchrotron radiation) or interaction with visible photons (Thomson scattering) is limited by, and comparable with, the shortness of the electron beam. for the AIS linac beam, the shortest photon pulses from a direct collinear interaction would be a few tens of picoseconds long. We have recently hypothesized that a third approach could break through this limit, protucing sub-picosecond $x$-ray pulses.

The new approach, being supported with I aboratory Directed Research and Development funds, is based upon 90 (ompton scattering with a visible laser (Figure 4-4). In this configuration, the shortness of the x-ray pulse is limited not by the length of the electron pulse, but rather by the length of the laser pulse or the transit time of the laser pulse across the waist of the focused electron pulse. Therefore it is crucial to focus the electron beam to a narrow waist matching the laser pulse length. A short-pulse solidstate laser $\left(\tau_{1}=200 \mathrm{fs}, \mathrm{E}=100-20() \mathrm{m}\right)$ ) is being designed by the femtesecond laser laboratory in I.BI's Materials Sciences Division. Also being designed is an $x$-ray detector that offers femtosecond time resolution. In cooperation

\footnotetext{
"The terms "overdense" and "underdense" indiente whether the plasma is denser than the particle beam or vice versa
} 


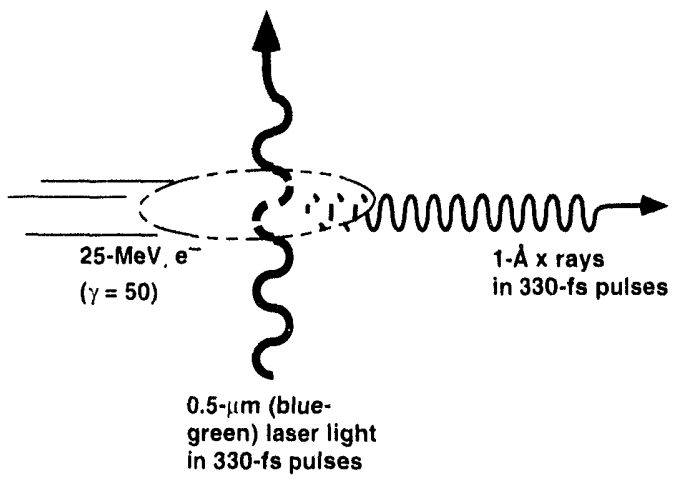

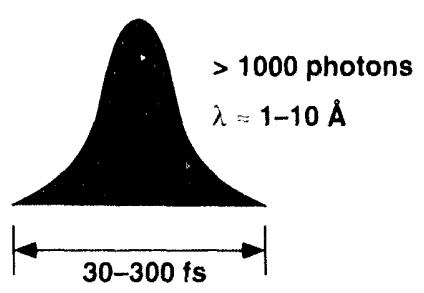

XBL $931-4708$
Figure 4-4. One of the most intriguing experiments for the Advanced Test Beam Facility is production of sub-picosecond $x$ ray pulses through $90^{\circ}$ Compton scattering of a visible laser beam against the 50-MeV electron beam.

with LBL's Center for X-ray Optics, we are examining ways to direct the beam onto detectors and experimental apparatus. We are also designing the beamline components required to focus the electron beam to a 70-100 $\mu \mathrm{m}$ spot and then separate it from the x-rays after the interaction point. With the current design parameters, we should be able to produce a 100-300 fs x-ray pulse, containing about $10^{5}-10^{6}$ photons, with a wavelength that can be varied in the range of $1-10 \AA$ by changing the electron-beam energy.

A variety of other experiments will also be made possible by the facility, including beam-structure interaction studies, investigations of beam-conditioning cavities for FELs, and the "chirping" of conveniently long (10-ps) electron-beam bunches to produce photon pulses much shorter than that.

Figure 4-5 shows the overall layout of the Test Beam Facility. We have been designing the beamline components while studying the proposed experiments and deciding how best to reconcile their somewhat different implications for the magnetic lattice of the transfer line. For example, the plasmafocus experiment requires several transverse measurements of the electron beam (which therefore should be perturbed as little as possible downstream of the focus), whereas the Compton-scattering experiment requires separation of the electron and $x$-ray beams downstream of the interaction point.

We anticipate that most experiments will be entirely transparent to ALS operations, involving no changes in the electron-gun and linac settings. Some special experiments might call for temporarily changing the relative amplitude and phase settings of the two linac tanks; others might require the gun pulser and the grid voltage to be turned up to their maximum capacity in terms of charge extraction and pulse-train length. The linac will remain under the overall control of the ALS throughout.

\footnotetext{
'A term fur a small, mapid change in energy during a pulse, historically based in radio transmission of Morse conte.
} 
Optics Experiments

Figure 4-5. Because the ALS is based on a storage ring, the injector linac will be idle much of the time. This affords a highly cost-effective opportunity to develop a facility for beamphysics research. This diagram shows the probable layout of the Advanced Test Beam Facility, which is now moving from design into construction. The facility will support a variety of experiments, such as plasma focusing of an electron beam and the production of ultrashort $x$-ray pulses using $90^{\circ}$ Compton scattering of a visible laser beam off an electron beam.

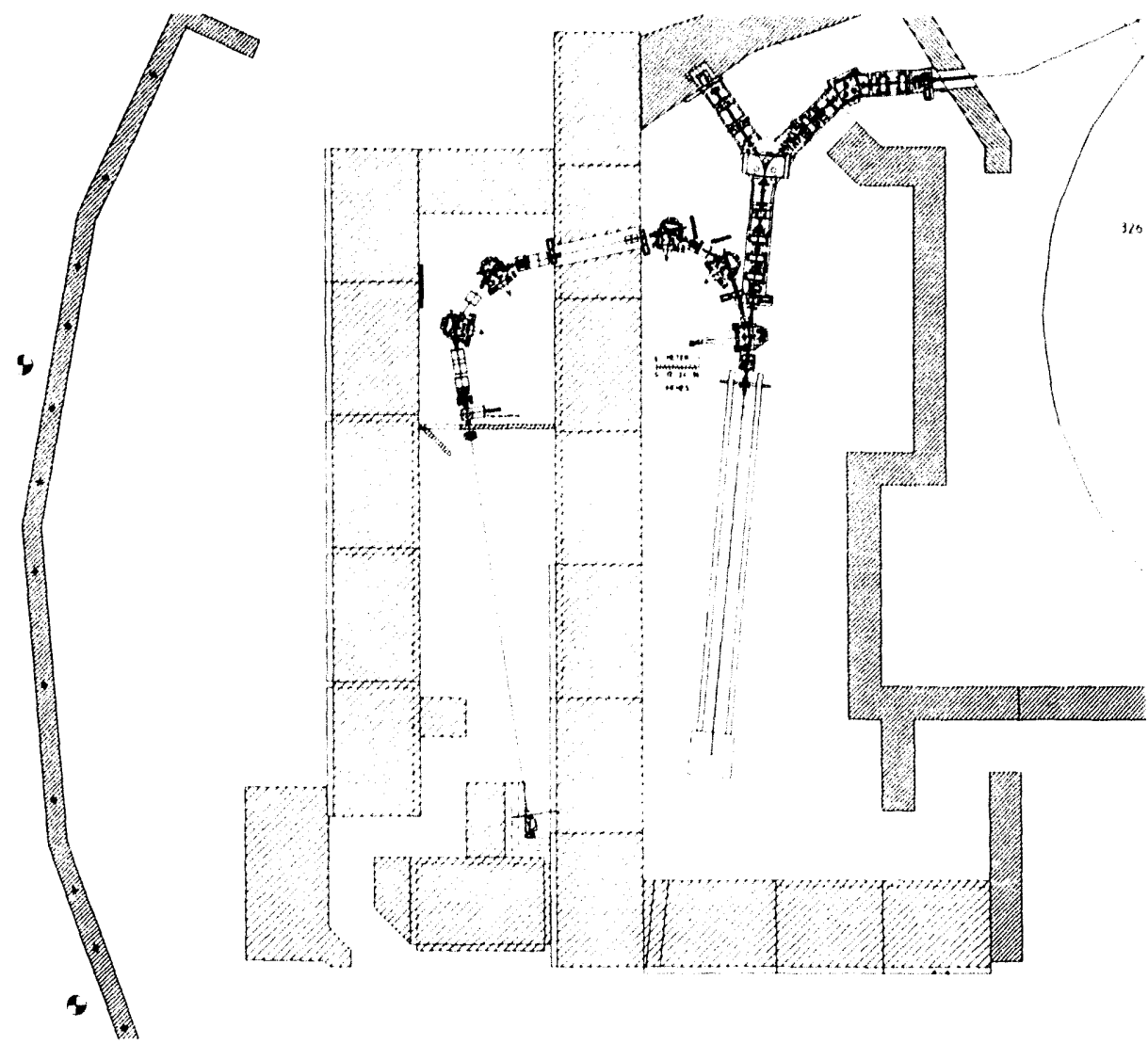

$X B L 9111.6852$

\section{Optics Experiments}

\author{
Bench Testing of HOl.D
}

The Center for Beam Physics has greatly expanded its experimental capabilities avith a newe optics laboratory that will serve many efforts, including the design of the' proposed CDRL. IRFEI and the development of optical components for the Te'st Be'an Facility described in the previous section. Teo projects have been carried out thus far: bench testing of the Fox-Li code Hom Hand development, together with Stanford University, of advanced optical diagnostics for FEL.s.

Hole coupling was selected for the CDRL IRFEL's optical resonator after extensive computer simulation, primarily using the code Hol.). To validate these simulations, we performed scaled experiments by injecting a visible HeNe laser beam into a stable cavity, as shown in Figure 4-6. We studied two cases: a Gaussian near-concentric symmetric resonator and a holecoupled resonator with degenerate higher-order modes. The first case, with its simple geometry, allowed direct comparison with analytical results and Hol.1) output and was also useful for benchmarking the diagnostic equipment. The second case provided an effective means of exercising the code and also yielded intrinsically useful results, since mode degeneracy should be avoided for good FEL operation. 


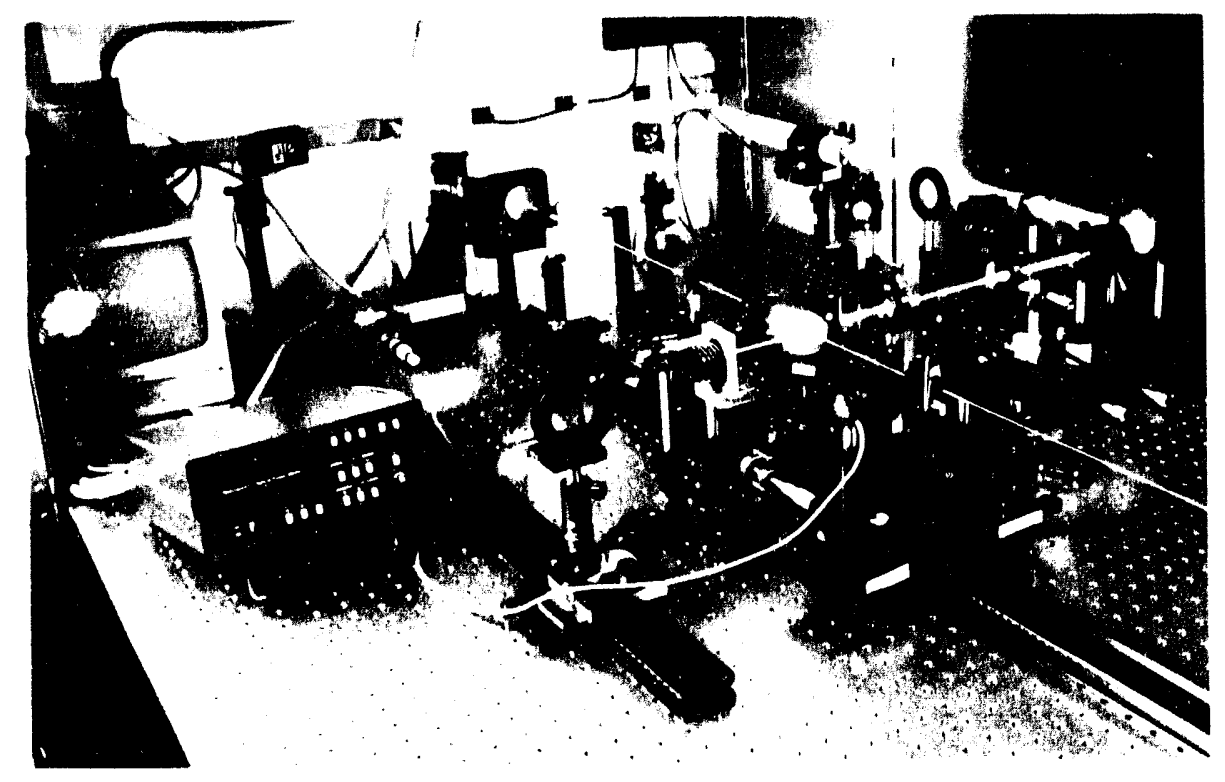

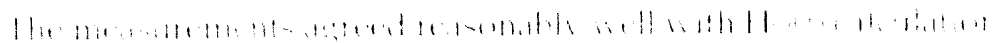

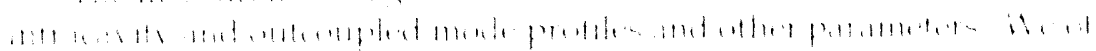

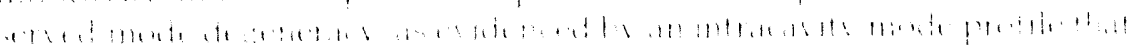

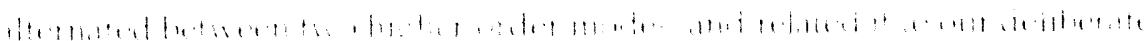

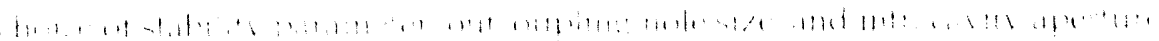

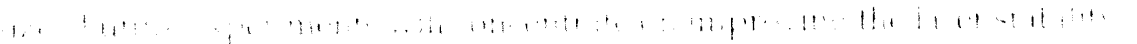

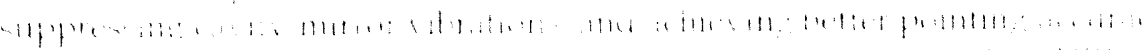

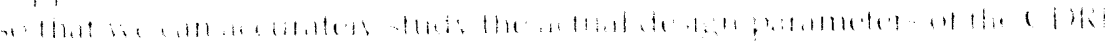
$1 k \mid 1$

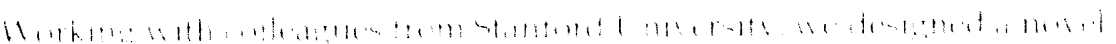

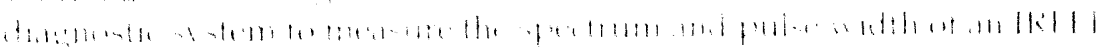

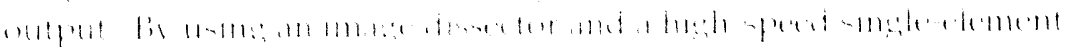

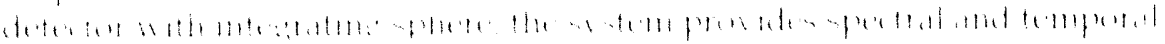



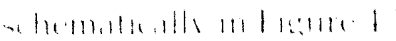

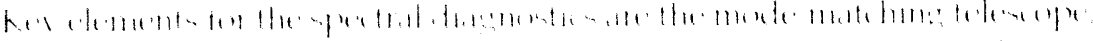

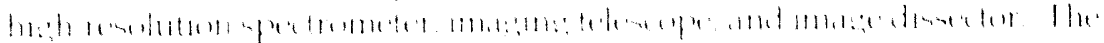

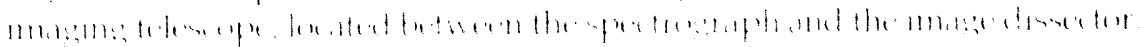

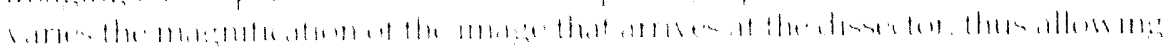

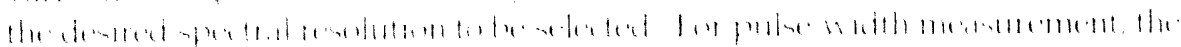

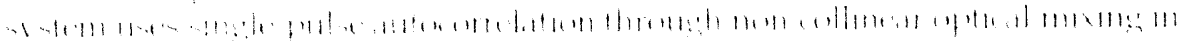

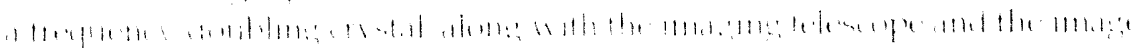

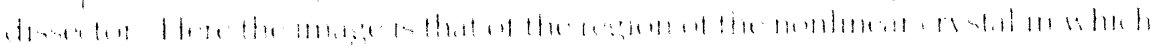

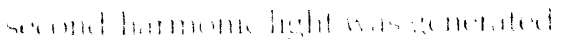

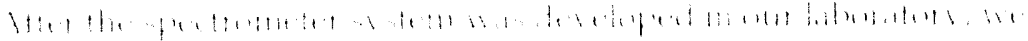

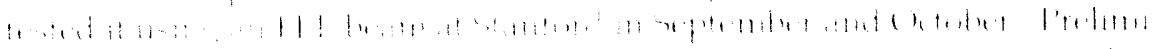

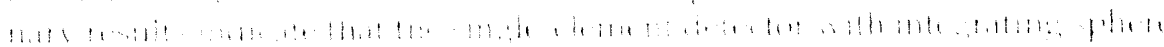

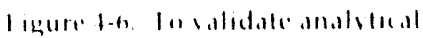

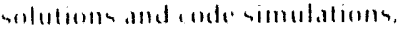
we pertermed saled experimente

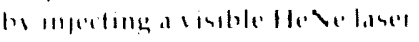

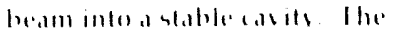
conls tor serits the lox-l i code

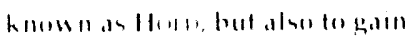

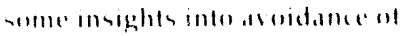
degenerale higher-erder moden in 1114

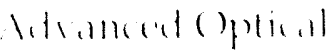

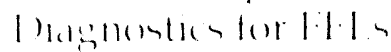


Optics Experiments

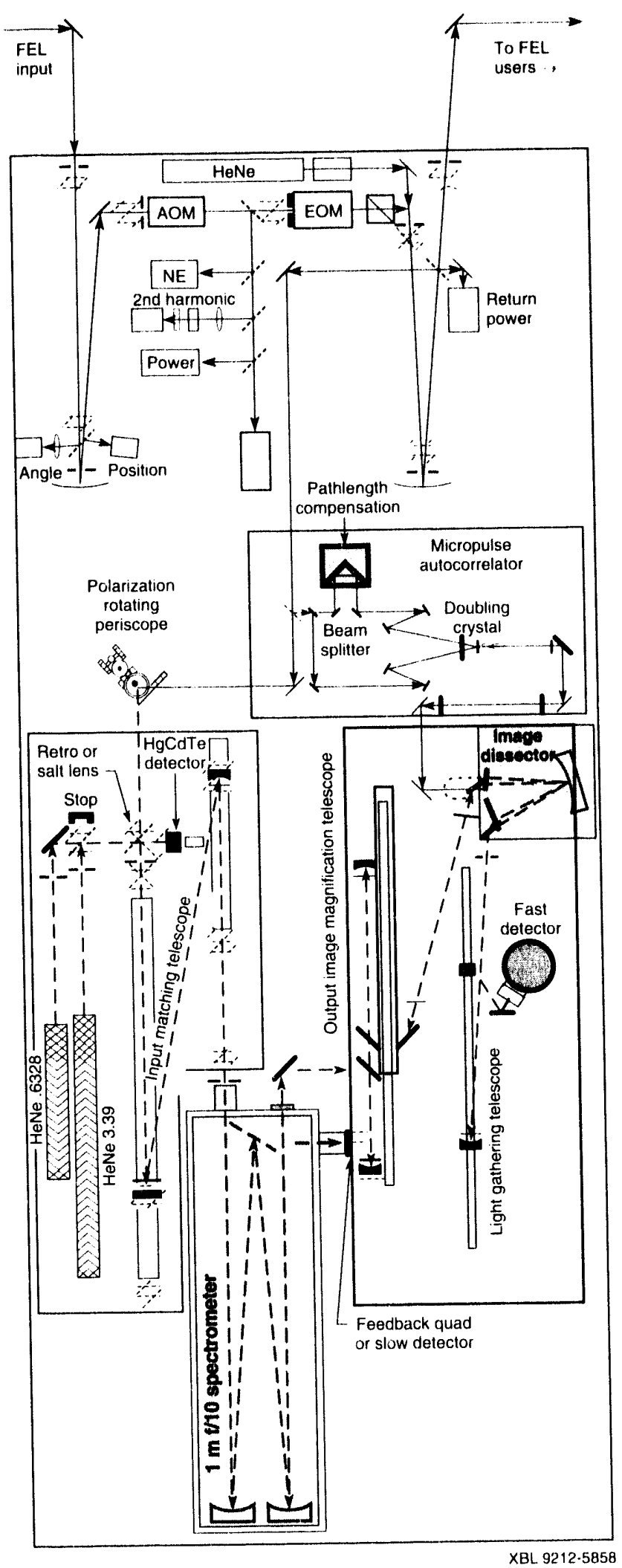

Figure 4-7. The LBL-Stanford collaboration in FEL diagnostics has resulted in a system that can perform both spectral analysis and pulse-width measurement of the optical output. Preliminary results from testing with an FEL beam at Stanford have been encouraging. 


\section{CENTER FOR BEAM PHYSICS}

has the requisite sensitivity and rise time. A micropulse spectrum with 5 spectral "bins," or sets of data in different parts of the spectrum, was obtained. Further improvements to the spectrometer and its user interface will be tested in another FEL run at Stanford early in 1993, and we are also about to begin building the single-pulse autocorrelator system for pulse-width measurements.

Members of the Conter for Beam Physics have heen indeled in the Adtanced light Souree from the outset, focusing primarily on the immed tinte needs of the propect but

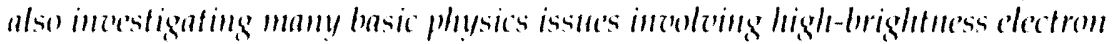
storage rings that howe' mumerous insertion derices. Much of this reserarch is highly ge'neric and is rele'ent, for the most part, to any third-generation solure', as we'l as to storage-ring-based fre'-eldetrom lasers and to compact damping rings endisioned for highte-nergy line'ar colliders.

Because of the high beam current and short bunch length in the ALS, it is important to minimize the beam coupling impedance of the vacuum-vessel components. If this impedance were high, it might excite coupled-bunch and single-bunch instabilities, and the electromagnetic energy deposited in the vacuum vessel by the beam (hundreds of kilowatts are potentially arailable) might cause excessive heating. Our Beam Electrodynamics group has studied many ALS structures, beginning at the design stage, a process that includes computer modeling and measurements of some actual components by launching waves down a coaxial wire. Our most recent achievement was measuring the higher-order modes of a spare AIS rf cavity. With these measurements we determined the effectiveness of damping (which is accomplished with high-pass filters connected to the input power waveguide) and obtained the data necessary for accurate simulation of coupled-bunch motion.

The information is being put to use in the design of damping systems. In the ALS, bunch-by-bunch damping schemes will be implemented for all three axes so that errors in the position and phase of each bunch can be measured and corrected. Computer simulations and calculations of the beam behavior, which used the data on the higher-order modes of the rf cavity, suggest that the beam instabilities can be safely contained with the proposed feedback systems. Tests of the longitudinal feedback system are planned on the ALS in the near future. This system was developed in cooperation with SLAC, and a similar scheme will be used for the PEI'-II collider described earlier in this chapter.

The device that will apply the feedback to the beam is a "kicker," which consists of a pair of coaxial electrodes approximately a quarter-wavelength long connected by half-wavelength delay lines. This structure uses only onefourth the power of a single-electrode design for a kick of the same amplitude. Low-power measurements of a prototype confirm the predicted performance, and a production unit is being made. Transverse kickers are also being designed.

\section{Accelerator Physics for the ALS}

\author{
RF Measurements and \\ Feedback Systems
}




\section{Injector Commissioning Experience}

\section{Beam \\ Electrodynamics}

Members of our Center working on the ALS project have been closely involved in the commissioning of the 5()-MeV injection linac and the $1.5-6$ ieV booster synchrotron. This process (see (hapter 3 of the AFRI) Sammmary of Actirities) is esentially complete; we are now studying the beam dynamics of the injection system and taking as many opportunities as possible to gain operational experience with it. By building our understanding of this complicated system, we will be able to commission the storage ring efficiently in early 1993 and then provide smooth, reliable operations after the facility upens to users.

As greater demands are mate on the performance of actelerators-sach as increased

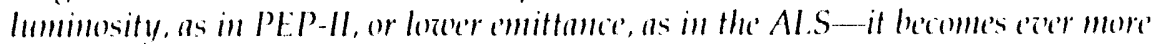
important to canderstand potentially dismption of pheneme'na abithin the beam chamber and to perform inrious of "gymmastics" to monitor and control the beam. An area of special interest is the umiterstanding and control of the potentially

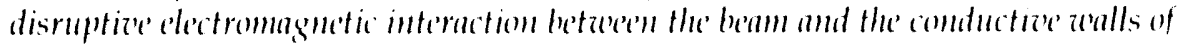

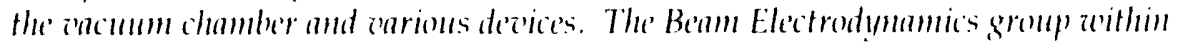
the Conter approaches the'se problems through analysis, simblation, and experime'ntation. In 1992 they contributed to PEP-II by leading the de'sign of rifand fered and

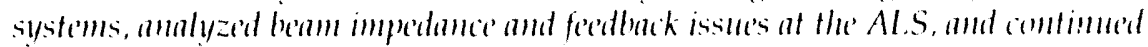
the ir history of contribution to the Trobtron by statying a stochastic beam-conting ingrale.

The major rf-design challenge posed by PEP'-II is control of coupled-bunch motions. In each of the three directions (horizontal, vertical, and longitudinal), these motions have 1658 modes that may be driven strongly by the higher-order resomances of the rf cavities. Each higher-order cavity mode can drive a hundred or so of these motions at a growth rate thousands of times faster than the damping that naturally occurs in the accelerator. The first step toward stabilization is to reduce the shunt impedances of the higher-order modes by several orders of magnitude without corresponding degradation of the desired fundamental mode. Removal of the remaining instabilities will then be within the reach of a practical feedback system. To reduce the shunt impedance of the higher-order modes, we attach waveguides to the cavity to couple these modes to an external resistor.

Figure 4-8 shows a design for such a cavity and a low-power prototype, designed and analyzed with the aid of the MAIA code and Kroll-Yu processing of the output. Extensive measurements designed to examine which modes are damped and whether there is interference with the fundamental mode have shown good agreement with expectations; for example, the strongest longitudinal mode, $\mathrm{TM}_{0 H}$, was predicted to have a loaded $Q$ of less than 30 and was measured to have a loaded $Q$ of approximately 28 (suppressed by more than three orders of magnitude from the unloaded case). We developed a bead-pull perturbation apparatus to measure the impedances of the cavity modes and to map field profiles for mode identification. We are now designing a high-power test model to verify fabrication and 


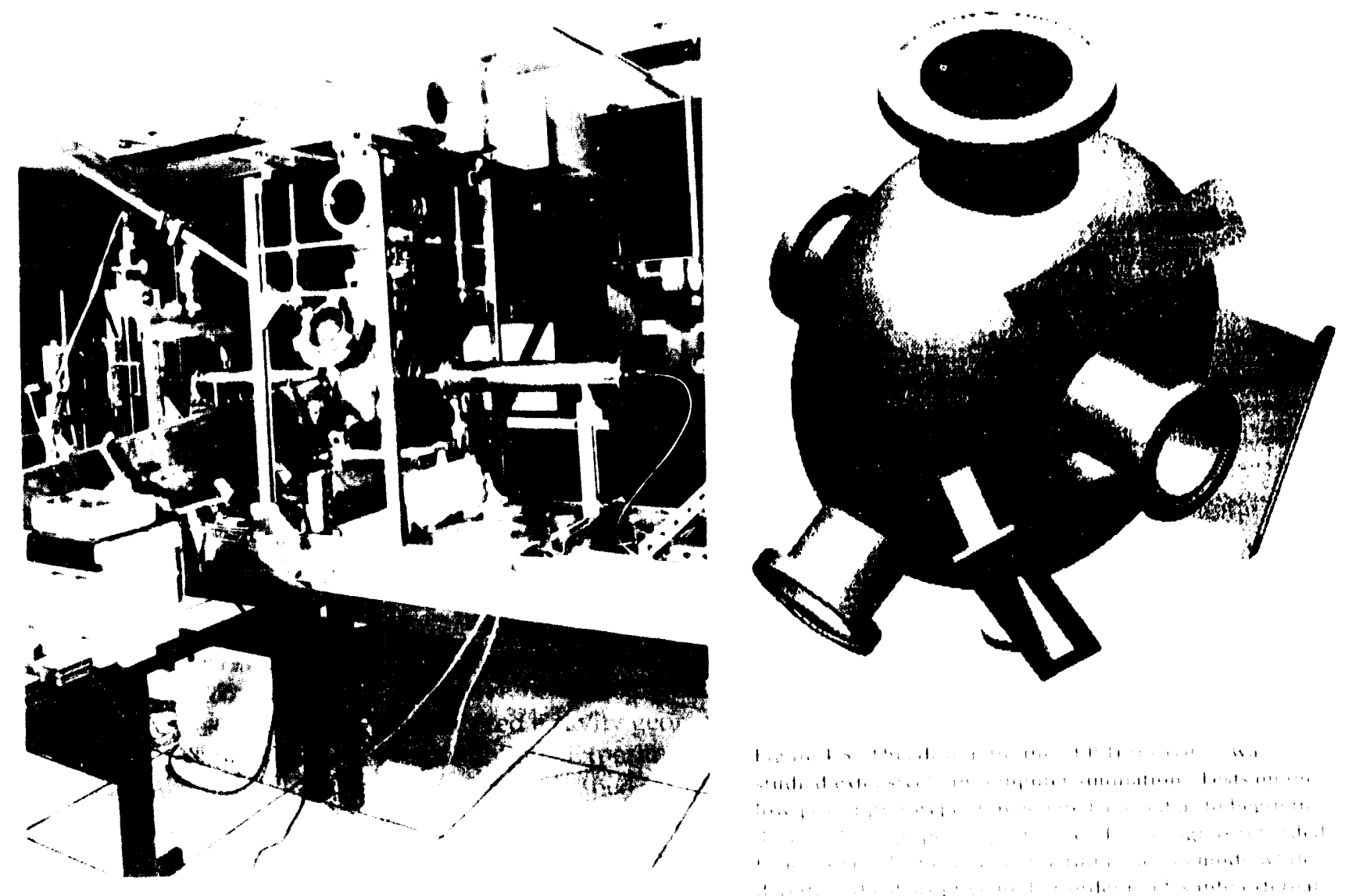


Fermilab Antiproton
Cooling System

FEL Accelerating Cavities
The latest acherement in our ongoing collaboration with liermilab is the design of a biplanar electrode system for more rapid cololing of the beam for the antiproten soures. (I. BI. Was involved in the initial design of pickup and kicker electrodes for this cooling system and has berel comtinually engaged in analyzing the systemi's performance and seceking ways of improving it.)

In carlier vears we had demonstrated that, for power-limited conding systems, it is more efficient and cost-effective to double the number of cooling electrodes than to double the operating freguency, whereupon we develeped biplanar alectrodes that combleffectively double the number of clectrodes without using any more space. This scheme, with the existing 2-4 Cittz clectronics, appeared to yield better results than would a system with uniplanar electrodes and completely reworked 4-8 (ill/ alectronics. (alculations indicate that the resulting performance would exced the needs of any anticipated upgrade to the Tevatron complex, including the propesed new main injector. The validity of our heam-cooling calculations was alfirmed by comparing the results with conling data from Fermilab. In 1992, we completed a detailed design and cost estimates for the electrode system and began studying the performonce of a prototype module. full production awaits the results of the study and a go-ahead decision by fermilab.

As mentioned in the carlier section on the propersed (I)RI. IRFFL, minimi/ation of fluctuations in the energy and intensity of an l:1:L requires stringent maintenance of electron-beam stability. This in turn has implications for the rf cavities in the acelerator and ether aspects of the accelerating system. For instance, coupled-bunch motions driven by the higher-order modes of the rf cavity may be excited in a recirculating beam, causing rf voltage fluctuations that make the energy of the beam fluctuate. As part of our collaboration with Stanford, Brookhaven, and TRW, we have performed various experiments in FEl. technolugy.

A two-cell superconducting rf cavity, similar to a cavity of the IRFII, has been studied extensively with our bead-pull perturbation apparatus in search of higher-order mode's. These modes can be damped by putting probes at appropriate lecations in the beampipes at the ends of the cavity and comnecting them to external resistive loads. A similar copper cavity has been used to study a network for input impedance matching and phase adjustment. In 1993, together with Stanford, we will measure the yuality factors of the higher-order modes of this niobium cavity at silperconducting temperatures.

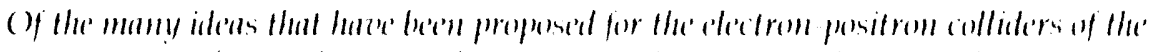

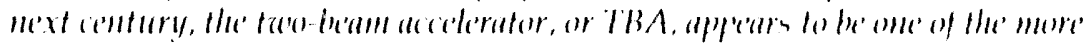

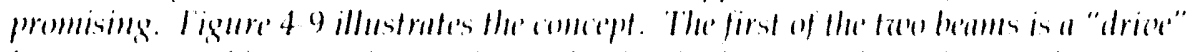

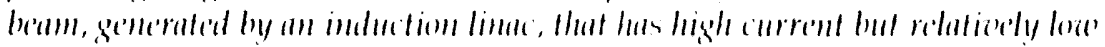

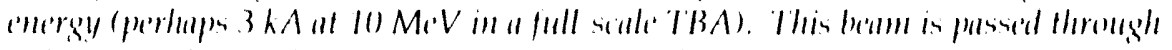

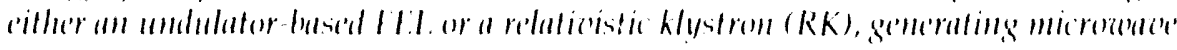

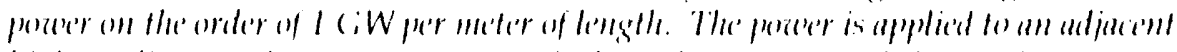

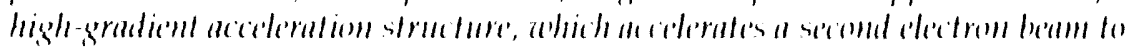

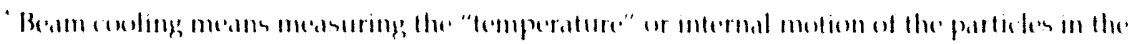

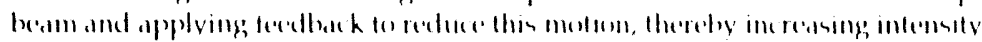




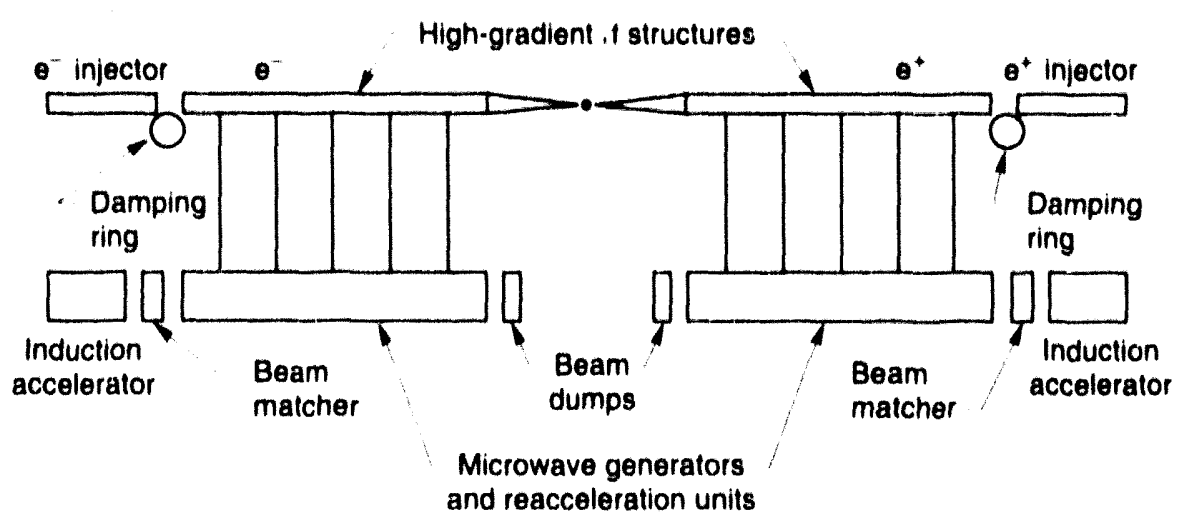

$\times B L=901.5730$

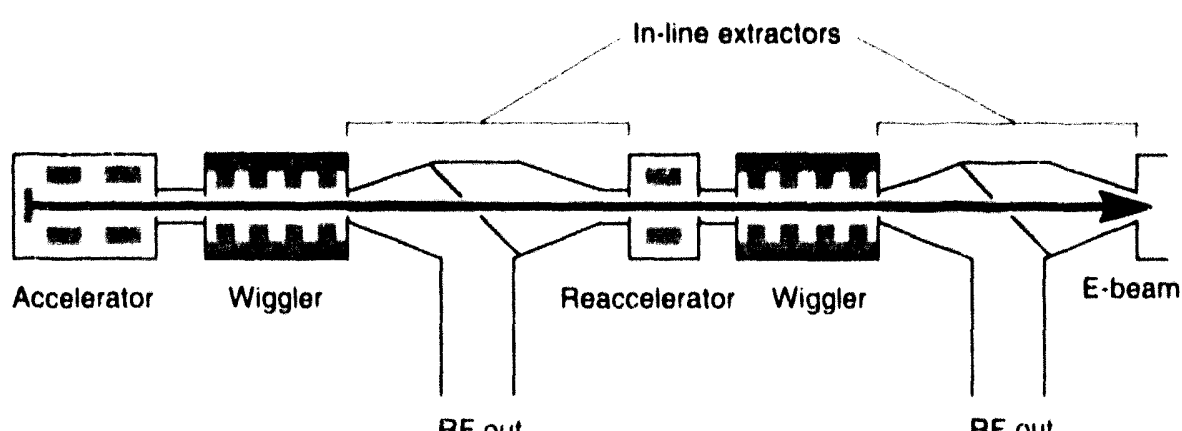

RF out

RF out

×8. 901.5729

high energy. Today, the TBA techology is in the early stages of development: designs are being developed and evaluated by researchers in the Conter's High. Energy Collider Physics Group, in collaboration with collengue's from LLNL and from KE.K, the highte'nergy physics laboratory in Japan.

Initially the TBA/RK work involved longitudinal bunching of the drive beam. This is adequate for low energies, but at moderate energies (greater than $3 \mathrm{MeV}$ or sol) it becomes less effective. To extend our work to higher energies, we have been experimenting with a transverse chopper cavity or "choppertron," built according to our designs by Haimson Research. In 1991 trials, the choppertron produced impressive peak power-some 400 $\mathrm{MW}$-but the pulses at such power levels were less than $10 \mathrm{~ns}$ in length. We determined that the problem was beam breakup caused by a spurious higher-order mode generated in the output structure at $13.6 \mathrm{GHz}$. We have since added a damping structure (Figure 4-10) that removes this higher-order mode; in 3-MeV experiments on the Advanced Technology Accelerator at LLNL, we obtained 30-ns pulses at $120 \mathrm{MW}$. These pulses had a phase jitter of about $2^{\circ}$, which implies good spectral purity. This satisfying demonstration of high power output from an RK provides a good basis for our continuing R\&D program. We are investigating damping structures that could remove the higher-order mode without damping the desired mode, perhaps enabling us to simultaneously achieve the hundreds-of-megawatts power and the tens-of-nanoseconds pulse lengths.
Higure 4-9. As shown in the TBA sketch abote, a high-current, low. energy drive beam is used for generaling if power that applied to a high-gradi .tl acceleration structure, where a low-current load beam is accelerated to high energy. The diagram below' shows the progress of the drive beam through the rfgenerating devices (FEL wigglers in this examplel and the reacceleration units that replenish the drive beam in between.
Transversely Modulated RK 
$11.4 \mathrm{GHz}$ output

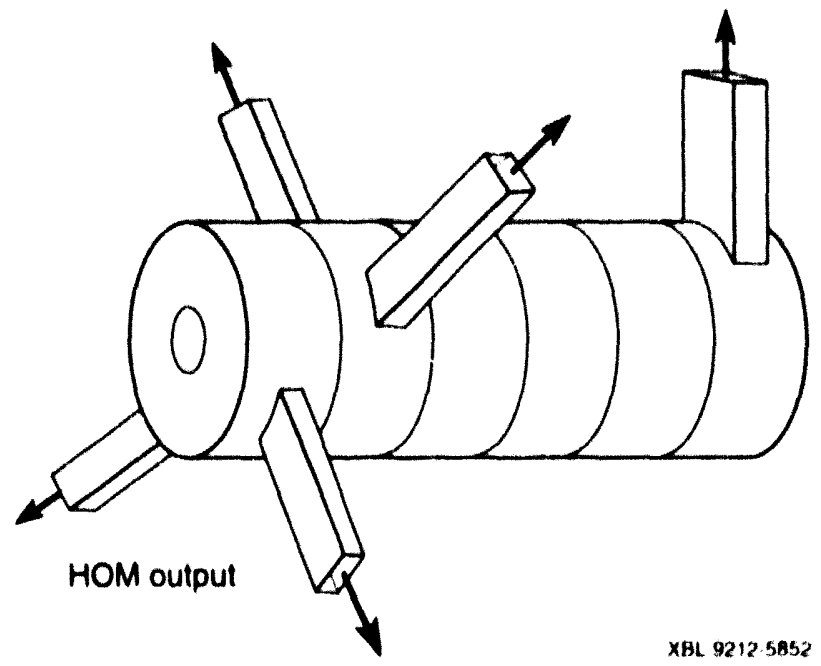

Figure 4-10. When the choppertron was equipped with this structure, a highly disruptive higher-order mode was effectively damped, enabling a 30-ns pulse of 11.4 GHz microwaves at 120 MW. The low phase jitter (about $\$ 22^{\circ}$ indicates good frequency stability. The next step is to improve the efficiency of the damper so that the power level in the fundamental mode returns to the hundreds-of-megawatts level achieved (in much briefer pulses) withoul the damper. (After LLNL illustrations)
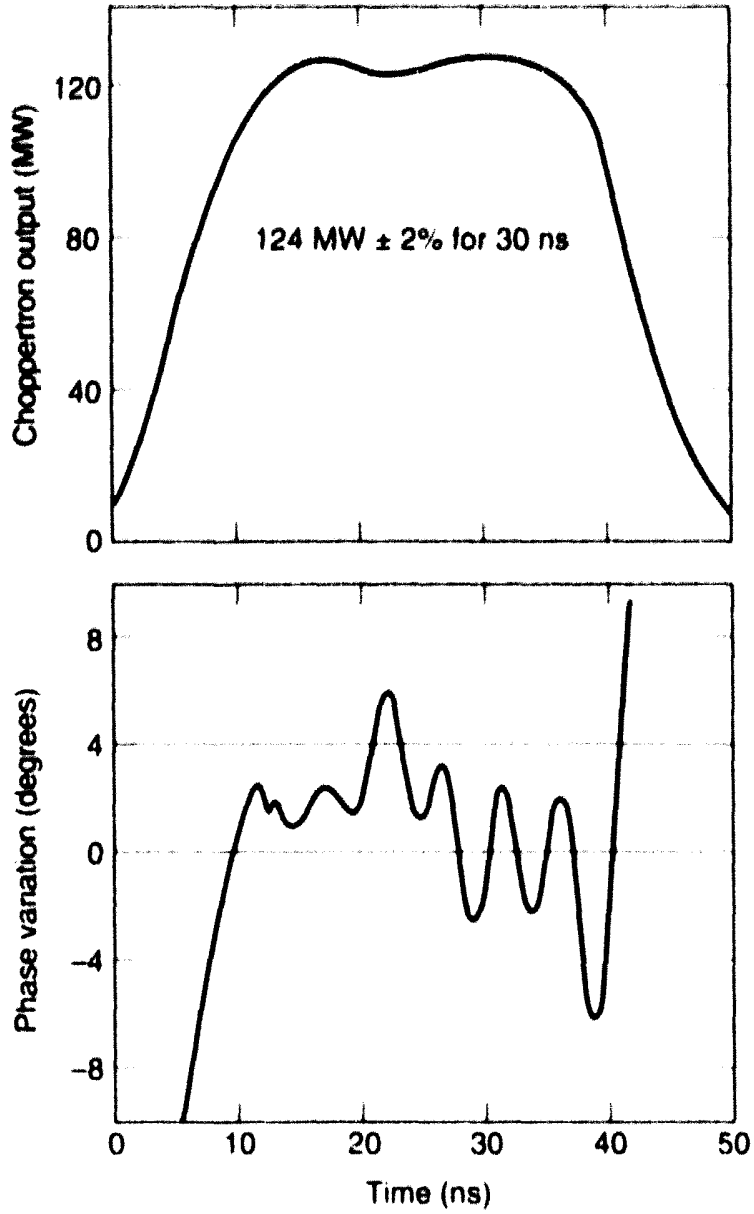

XBL 9212.5852
Standing-Wave FEL

Horizons for the TBA
The FEL., explored in our original TBA research, remains a proven candidate with great potential. We are developing an idea, called the "standing-wave FEL," in which the radiation is trapped in a standing-wave rf cavity and beat-coupled to a nearby high-gradient acceleration structure. This concept is currently undergoing intense analysis and theoretical study, with no experimental program anticipated in the near term.

The work done on the TBA since we conceived of it 10 years ago has validated the basic concept and the use of either an RK or an FEL as the source of rf power. However, there remains a vast amount of research and development before the TBA can be used in high-energy physics. Here are some of the planned near-term investigations.

- Re-acceleration of the "spent" drive beam (useful for economic reasons) will be examined in a planned 1993 experiment at L.LNL.

- There is much theoretical and experimental work to be done in extraction of microwaves from the power source. A demonstration of repeated extraction is being studied at LL.NL. 


\section{( INTER IOR BHAM IIIYSIC}

- Sensilisity atudien fo determine the importance of various parameters will be important. A great deal on theoretical work has been done; coming veors will aece mure sledien an real apporallus.

- Coonomic motters will be significiont in the eventesl decision on whether to build a full-ecalde tBA and in the fechnolegical cherices within such a project. We are working with II.NI on these isstles

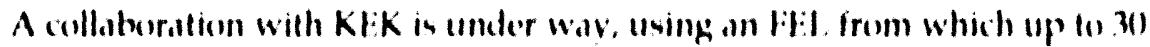
MW cant be entrocted at s.h (ille.

The gain of a free-electron loser or other renomant electron-beam device is limited by the spread in longitudimal velecity and, hence, the energy spread and enittance of a threedemensional beam. The electron-beam emittance must be less than the wavelength of the radiation from the device divided by $4 \pi$. In pratice, the energis uprend of the beam is often small, so the beam could be "conditioned" with spectal rf covitien. These cavities would impart more acceleretion to the particlen troveding longer pathe, reducing the spread in lengertulimal velocity. We have analyad this idede with a simple numerical model for beam tromsport, assuming ideal rf covitien. We have also allalyad

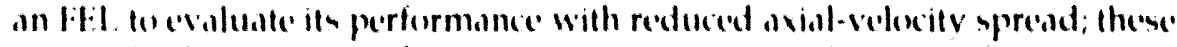

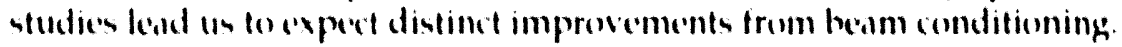
During 1492 we computationally anoly/ed a colvity feremetry that promisen to greatly increase the heam-colvity coupling. Baperiments fo test the feasibilitv of a beam-conditioning covity are being planned for the Accelerater fenst Facility at Browhhaven National laboraltory.

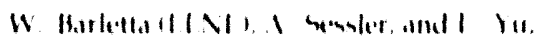

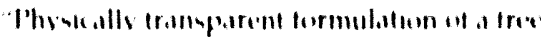



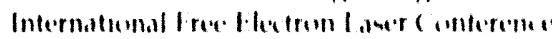

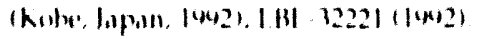

W Marrs.1 Hoghefter and

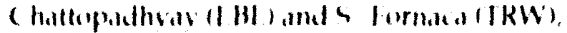

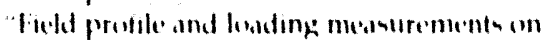

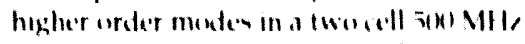
supercontulusting structure," Inth

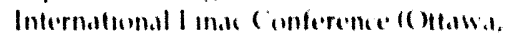
()ntario, (andda, 1442), 1 131 - 32178, (1942)

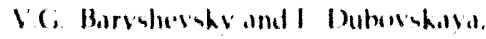
"Diffraction phenomena in spentaneosus and stimulated radiation be relitivistle particles

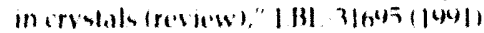

1. Ben-lii, I II Yu, K Cinvil, and AM heselet. "A propused experiment for beam conditionenge." It th Intermational f ree Electron laser conterence (Kobes, Japan. [42); Bronkhatren Nitionall laboratury report BNI $47 \times 57(1+42)$

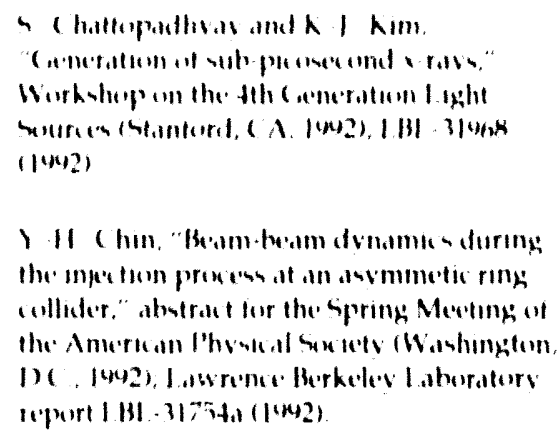

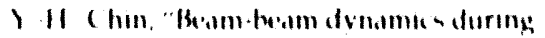

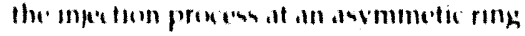

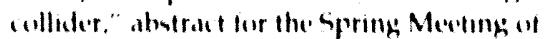

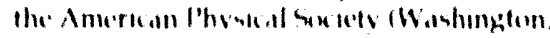

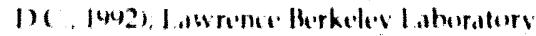
nepert I 131. $3175+11(1+42)$.

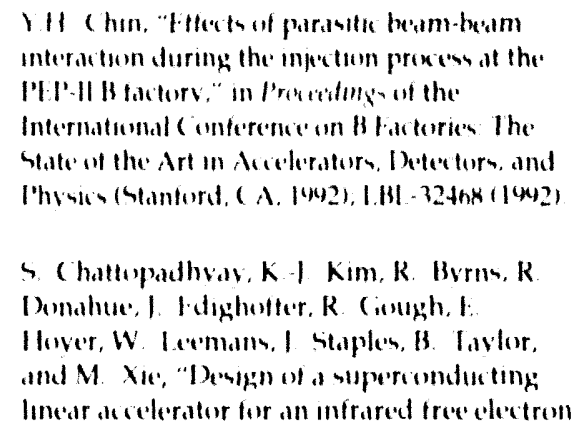

Beam Conditioning

Publications and Presentations 


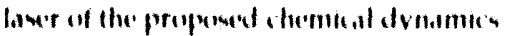

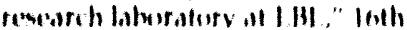

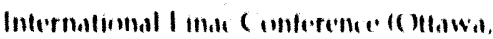

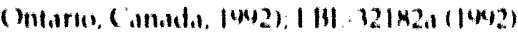

Yll Chon, "simple furmuline fin the

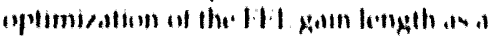

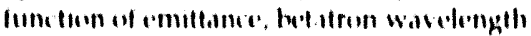

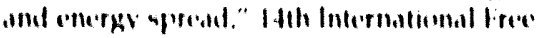

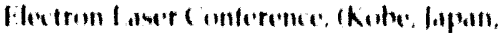
$(x) 2), 1131 \cdot 2244(1)(x) 2)$

Yll (hin, $\mathrm{k}$ I $\mathrm{kim}$, and $\mathrm{M} \mathrm{X}_{\mathrm{II}}$

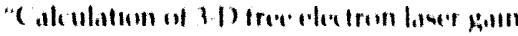

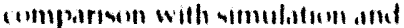

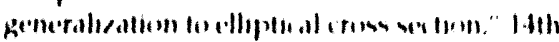

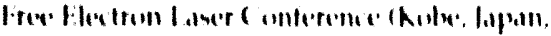

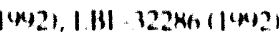

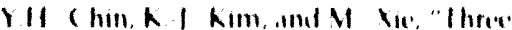

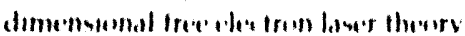

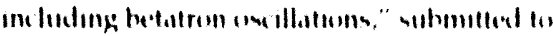

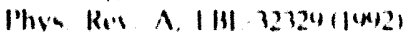

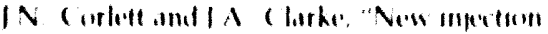

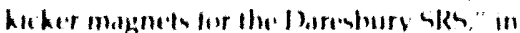

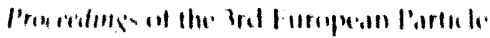

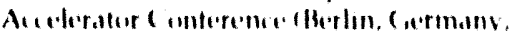

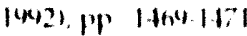

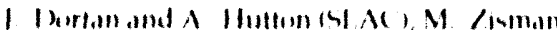

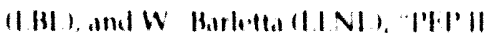

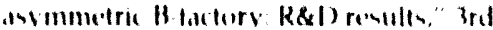

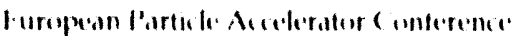

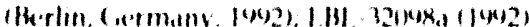

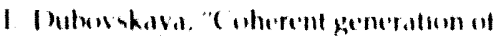

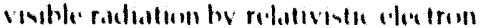

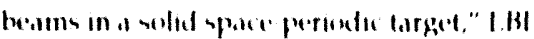
$\| 16,4+|(x)|)$

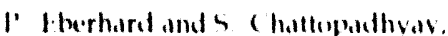
"Anvmmetric phi lecterren a propened

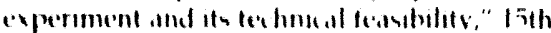

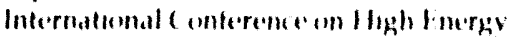

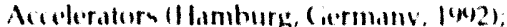
I 131. $3225(1)(1442)$

1. Forest andk Ilirata, "A contemporars

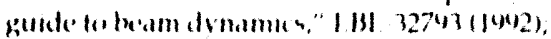
iles publinhed he klk (164)

1. Forent andk (1)mon, "somplecte

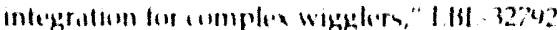



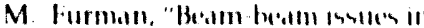
anvmmetris colliders," invited talk, in

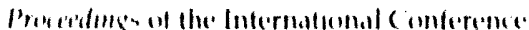

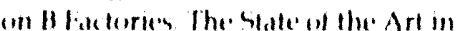

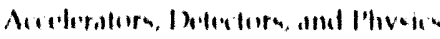

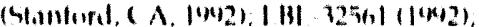
invited lalk, spring Meeting of the Americin

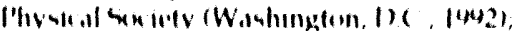
IIII $313921(1002)$

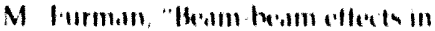

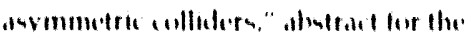

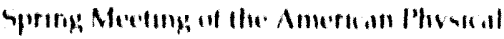

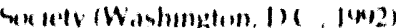

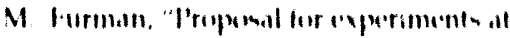

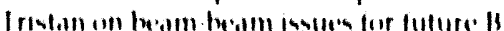

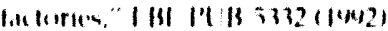

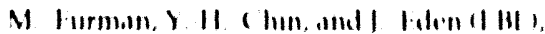

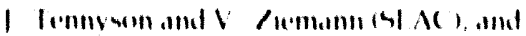

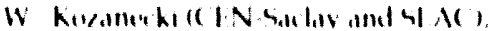

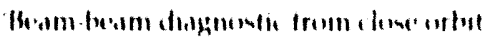

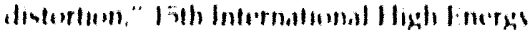

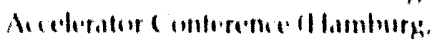

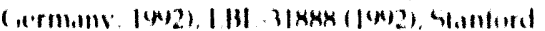

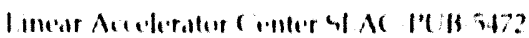
$(1 \ln 2)$

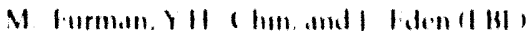

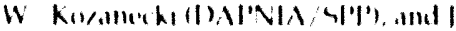

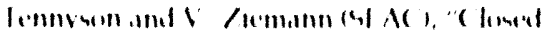
urbed denterteren and the berim heam

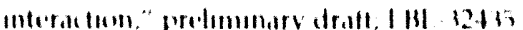
$(1+x) 2)$

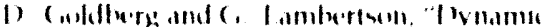

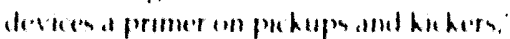
chopter in All' Conference l'berededinges

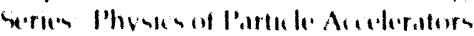

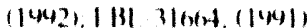

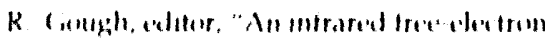

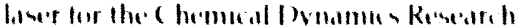

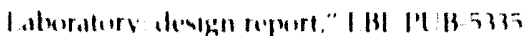
(1) wen)

K. Coril RA Kimmer. and A Sender

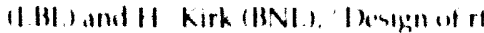

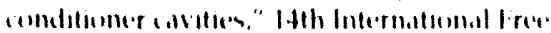

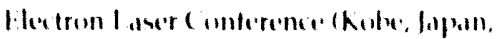
$(4+2) \mid 131.22272(1)(x) 2)$

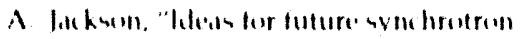

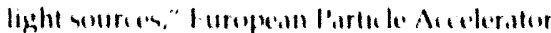

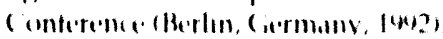
$1111 \cdot 31172110021$

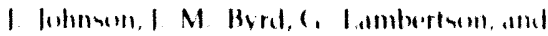

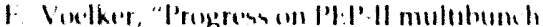

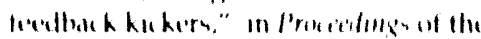

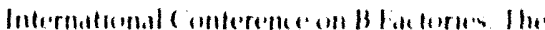

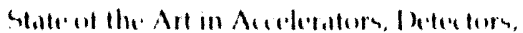

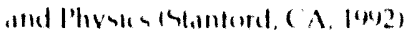




\section{CHNTER HOR BEAM INIYSICS}

K. I Aim. "Cilculation of perak power for short wavelength Hill s," Workshopon the

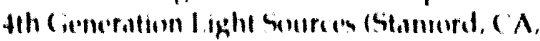

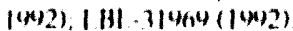

K.1. Kim, "symmetrle and s summetric multiperiont wigglere in leng: wavelengtl limit." suhnutted to Nuct Instrum Mith. $1.131-31249(|(N)|)$

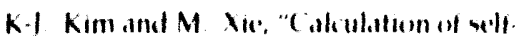
amplitied pountaneous emission in the short

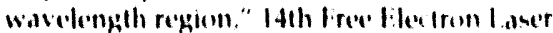

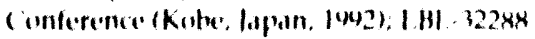
$(1(x) 2)$

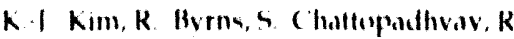
Domohuse, I folighofter, $R$ (oough, Ilover, W. Iermans, I Staplen, B Tavlor. and M. Xie, "An introred free clectron liser svatem for the propened ( hemicoll Bunamice

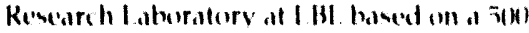

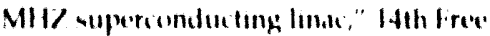

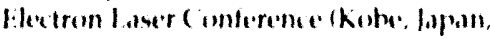
$1442), 1131.322 \times 7(1042)$

4 Krishongenple, "Coherent beam beam smulatiens," atoutract fer the spring Neceting:

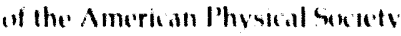

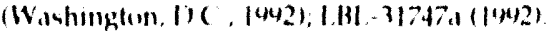

4 Krishnagopaland R.II Siemann, "An investigateon of coherent yuadrupele beam. beam eftects," 15th Intermathenal conferemes an lligh Energy Acceleraturs ll lamburg, (iermanv, 1(4)2): 1 B1-32581 (1402)

S Krishongeppol and $\mathrm{R}$ Siemann, "Seme" anpecten the two heom performance of IX I," submilled to Nucl Instrum. Meth: Stanford l inear Accoleratur Center Sl.AC

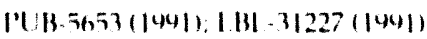

4 Krishnagopal and Y. II Chn, "Bench marking beam-beam simulations using coherent yuadrupele elfects," in l'resedens" af the Internattonal conterence on B Fiderenes the State of the Art in Accelerators Detecturs, and Phesses (Stantord, $C A, 1402$ ). I.1BI. $-32516(1442)$

5. Krishnagupal, (i. Rangorralan, and A Semoler, "The nulti-cavity freve-enectron laser," It th International firee filectron laber conterence

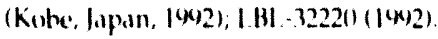

5. Krishnagopal, M. Xie, and K.-1. Kim, "Suppression of mode-beating in a soturated hole-coupling W1 oncillator," 14th International frece Electrun Laser conterence (Kube, lapan, 1942); 1.181-32223(1942)

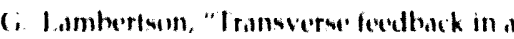
l(k) reV storage ring," in Procedense of the 19t1. Workshup on Maximising I uminusity

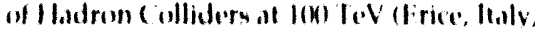

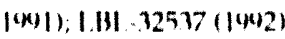

W. Icemans, I Rdigholfer, $k$ I Km, ands ( hattopadthoav, "Novel terheniguen for single pulse apectrum and pulsewidth meinuremente for an IR Fil.," I th Free Ilectron laser Conference (Kolde, lapan. $1042): 1.131 .222 \times 4(1042)$

W. Leemans, I Fighoffer, M. Xic, K I Kinn, ands. (haltopadhyay, "Hench-testing of IRIIII hole-coupled resonator designs using a ( W. IleNe lawer mode profile and intcoupling efficiency." 14th free llectron l.aner Conference (kitue, lapan, lw42), I.BI $22285(1(x) 2)$

(i. Romganrajon and A Sesoler, "Sensitivity studien of a standing wave free-edectron laner," Therd Workshepen Advanced

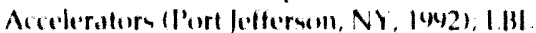
$32463(1492)$

K Rimmer, D) (ioldberg, (i I ambertson.

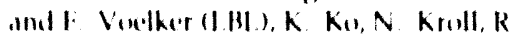
lendellen, and If hehwore (s) $A()$, and $N$ F. Alame and $M$ de long (AFC(1), "lligher order mode damping studien on the l'P:T' II B.

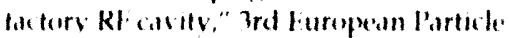


(w)2) 1.181. $323+4(1)(42)$

1. Si hachenger, "Summary of the working group on modelling and simulation," premented at the fifth Advanced If Fis Beam Donomion Workshep (Corpus Christi, TX. $|(4)|),|B|-3 \mid \ln (1)(\mid 04))$

A.M. Sessiler, "linde based VUV HII, review." abstract for the spring Meeting of the American l'hesical seciety (Woshington. I), 1002$) ; 1 . B 1-3169601(1092)$

$K$ lakavama, R. Covil, and A Sessler. "Macreparticle theorv of a standing wave ireenelectrom leser two-heam accelerator," submitted tu Nud. Instrum. Meth A; 131 $320111(142)$

C. Wang, "Conditioner tor a helically transpurted electron besm," Workshopon

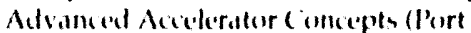
leffersen, NY, 1942); 1,131-32222 (1942)

\section{S. Wurtele, 1).H. Whittum, and A. M} Seanler. "Common andlysin of the relativistic klvatren and the slandeng wave free edectron 
Publications and Presentations

la r.er two-beam accelerator," 15th Inter national Conference on High Energy Accelerators (Hamburg, Germany, 1992); LBL-32580 (1992).

J. Wurtele, D. Whittum, and A. Sessler, "Impedance-based analysis of the relativistic klystron and the coupled-cavity free-electron laser two-beam accelerator," Third Workshop on Advanced Accelerators ('ort Jefferson, NY, 1992); LBL-31848 (1992).

M. Xie, "Variational analysis of 3-D FEL gain in beam distribution approach," 14 th Free Electron Laser Conference (Kobe, Japan, 1992); LBL_-32296 (1992).
M. Zisman, "B faciory rf system design issues," in Proceddings of the International Conference on B Factories: The State of the Art in Accelerators, Detectors, and Physics (Stanford, CA, 1992); LBL-32446 (1992).

M. Zisman (LBL), R. Bell, J. Dorfan, and II Schwarz (SLAC), and W. Barletta and $M$. Calderson (LLNL), "The PEP-II asymnetric B factory: design update and R\&D results," 15th International Conference on High Energy Accelerators (Hamburg, Germany, 1992); LBL-32559 (1992); Stanford Linear Accelerator Center SLAC: PUB-5852 (1992); Lawrence Livermore National Laboratory UCRL-JC-111380) (1992). 

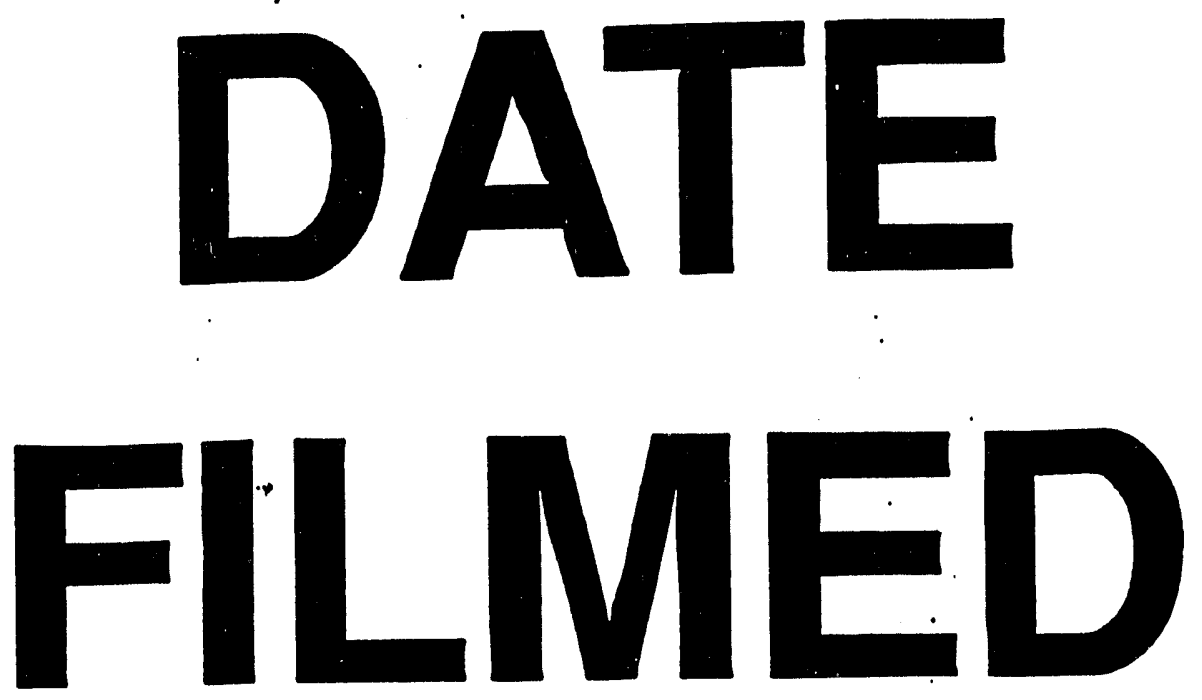

$12 / 29 / 93$
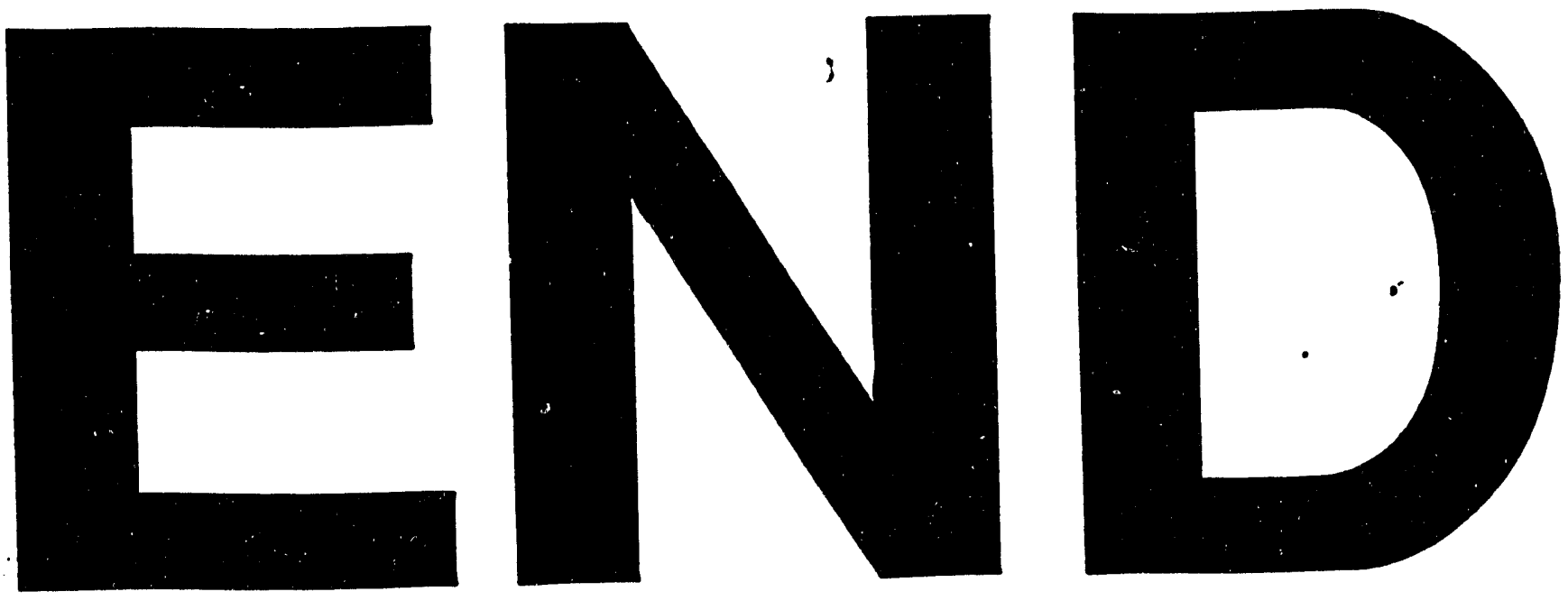


\footnotetext{
(1)
}

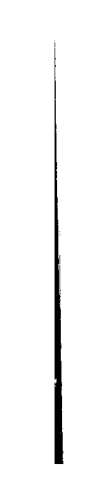

